Published in final edited form as:

Nat Genet. 2020 December 01; 52(12): 1303-1313. doi:10.1038/s41588-020-00725-7.

\title{
Genome-wide association study of intracranial aneurysms identifies 17 risk loci and genetic overlap with clinical risk factors
}

\author{
A full list of authors and affiliations appears at the end of the article.
}

\begin{abstract}
*Corresponding authors: Y.M. Ruigrok. ij.m.ruigrok@umcutrecht.nl and M.K. Bakker.m.k.bakker-25@umcutrecht.nl. Address: UMC Utrecht Brain Center, Department of Neurology and Neurosurgery, room G03-228. Heidelberglaan 100, 3584 CX Utrecht, the Netherlands.

$\dagger$ These authors jointly supervised the project

Ethical Statement

All participants provided written informed consent. The Biobank Research Ethics Committee of the University Medical Center Utrecht reviewed and approved the study protocol (TCBio 17-087). The following local data access and ethics committees approved collection and use of genetic data for this study. @ neurIST: Medisch Ethische Toetsings Commissie Erasmus MC (METC), Research Committee of the Hospital Clinic de Barcelona, Central Office for Research Ethics Committes (COREC) NHS, and Commission centrale d'éthique de la recherché sur l'être humain de la république et canton de Genève. ARIC: NHLBI Data Access Committee (through dbGaP). Busselton: GABRIEL Consortium Data Access Committee (through EGA). Utrecht 1: University Medical Center Utrecht Ethics Committee. Netherlands (EGA): Wellcome Trust Case-Control Consortium Data Access Committee (through EGA). Utrecht 2: University Medical Center Utrecht Ethics Committee. Doetinchem Cohort Study: Scientific Advisory Group of the Netherlands National Institute for Public Health and the Environment. Project MinE: Project MinE GWAS Consortium. French Canadian: Comité d'éthique de la recherche du Centre hospitalier de l'Université de Montréal and McGill University ethics. Finland (EGA): Wellcome Trust Case-Control Consortium Data Access Committee (through EGA). Finland: The ethics committee of Kuopio University Hospital and Helsinki University Hospital. NFBC1966: Ethics Committee of Northern Ostrobotnia Hospital District, Finland. ICAN: Institutional Review Boards (Comité consultatif sur le traitement de l'information en matière de recherche dans le domaine de la santé, Commission Nationale de l'Informatique et des Libertés) and Groupe Nantais d'Ethique dans le Domaine de la Santé (GNEDS). PREGO: Research Ethics Committee (CPP of Nantes). GAIN: NHLBI Data Access Committee (through dbGaP). FIA: University of Cincinatti ethics committee. nonGAIN: NHLBI Data Access Committee (through dbGaP). Poland: Institutional review board of the Jagiellonian University. NBS: Wellcome Trust Case-Control Consortium Data Access Committee (through EGA). UK Biobank: UK Biobank Data Access Committee. GOSH controls: Central London REC 3 committee. GOSH cases: Central London REC 3 committee. NBS+1958BBC: Wellcome Trust Case-Control Consortium Data Access Committee (through EGA). HUNT study: The Norwegian Data Inspectorate, the Norwegian Board of Health, and the Regional Committee for Ethics in Medical Research. China Kadoorie Biobank: Oxford University ethical committee and the China National CDC. Biobank Japan: Research ethics committees at the Institute of Medical Science, the University of Tokyo. More details can be found in the Life Sciences Reporting Summary.

Competing Interests

When this study was conducted, C.L.M.S. was chief scientist for the UK Biobank study.
\end{abstract}

\section{Author Contributions}

J.H.V. and Y.M.R. contributed equally to this study.

Writing and editing the manuscript: M.K.B., Y.M.R., J.H.V.

Supervising the project: Y.M.R., J.H.V.

Designing the study: I.C.H., S.D., B.B.W., J.P., A.S., E.I.G-P., M.N., J.E.J., M.v.U.Z.F.,

A.L., J.P.B., D.J.W., D.W., R.R., P.B., Y.K., J.H.V., Y.M.R.

Association analyses and scripts: M.K.B., R.A.A.v.d.S., W.v.R.

Functional analyses and scripts: M.K.B., R.A.A.v.d.S., W.v.R.

Phenotype preparation: S.M., R.B., C.M.F., S.H., S.S., J.D., O.M., P.B.

Technical assistance: R.A.A.v.d.S., K.R.v.E.

Phenotype and genotype contributions: M.K.B., Y.M.R., G.J.E.R., J.H.V., L.H.v.d.B., P.B., S.M., E.I.G-P., M.N., J.P., A.S., J.E.E., M.v.U.Z.F., A.L., G.A.R., S.Z., N.U.K., R.M., K.R., C.L.M.S., D.J.W., I.C.H., H.H., V.S.A., J.P.B., D.W., R.R., R.B., C.D., O.N., J.C.G., E.S., F.E., H.D., W.M.M.V.

Summary statistic contributions: Y.K., M.K., M.A., C.T., K.M. (BBJ), R.G.W., K.L., L.L., I.Y.M., Z.C. (CKB), B.S.W., S.B., M.B.J., B.M.B., M.S.S., C.J.W., K.H., J.A.Z. (HUNT), M.J.B., G.T.J. (AAA), H.K., J.G.Z., C.J.M.K., N.U.K. (AVM), D.W. (ICH), R.M., M.D. (IS), S.D., T.T., M.S. P.A. (cervical artery dissection).

Drug-target and MAGMA pathway enrichment analyses: J.R.I.C., G.B.

Critiquing the output for important intellectual content: D.J.W. 


\section{Abstract}

Rupture of an intracranial aneurysm leads to subarachnoid hemorrhage, a severe type of stroke. To discover new genetic loci and the genetic architecture of intracranial aneurysms, we performed a cross-ethnic, genome-wide association study in 10,754 cases and 306,882 controls of European and East Asian ancestry. We discovered 17 risk loci, 11 of which are new. We reveal a polygenic architecture and explain over half of the disease heritability. We show a high genetic correlation between ruptured and unruptured intracranial aneurysms. A suggestive role for endothelial cells is found using gene mapping and heritability enrichment. Drug target enrichment shows pleiotropy between intracranial aneurysms and anti-epileptic and sex hormone drugs, providing insights into intracranial aneurysm pathophysiology.

Finally, genetic risks for smoking and high blood pressure, the two main clinical risk factors, play important roles in intracranial aneurysm risk and drive most of the genetic correlation between intracranial aneurysms and other cerebrovascular traits.

An intracranial aneurysm is a balloon-shaped dilatation, usually located at a branch of an intracranial artery. It is present in $3 \%$ of the population ${ }^{1}$. Rupture of an intracranial aneurysm causes an aneurysmal subarachnoid hemorrhage (aSAH), a severe type of stroke. Approximately one third of patients die, and another third remain dependent for daily life activities $^{2}$. Intracranial aneurysms occur in relatively young people with a mean age of 50 years and is twice as common in women over 50 years old compared to men of that age. Genetic predisposition plays an important role in the disease with an aSAH heritability of $41 \%$, as estimated in a twin study ${ }^{3}$.

Much is still unknown about the genetic architecture of intracranial aneurysms ${ }^{4,5}$. Familybased studies identified a number of variants with Mendelian inheritance ${ }^{6-10}$, but genomewide association studies (GWAS) have identified multiple common variants, suggesting a polygenic model of inheritance ${ }^{5,11-13}$. The largest GWAS published to date, involving 2,780 cases and 12,515 controls, identified six risk loci ${ }^{11,13}$. Based on that GWAS, the explained single nucleotide polymorphism (SNP)-based heritability of intracranial aneurysms was estimated as being only 4.1-6.1\%, depending on population ${ }^{5}$.

We aimed to further characterize the genetic architecture of intracranial aneurysms by performing a cross-ethnic GWAS meta-analysis on a total of 10,754 cases and 306,882 controls from a wide range of European and East Asian ancestries. We included both cases with unruptured intracranial aneurysm and aSAH (i.e. with ruptured intracranial aneurysm), enabling us to identify potential risk factors specific for intracranial aneurysm rupture. We also looked for genetic similarities between intracranial aneurysms and related traits, including other types of stroke, vascular malformations and other aneurysms, and analyzed whether known risk factors for intracranial aneurysms play a causal genetic role. Further, we investigated enrichment of genetic associations in functional genetic regions, tissue subtypes, and drug classes to provide insight into intracranial aneurysm pathophysiology. 


\section{Results}

\section{GWAS of intracranial aneurysms}

Our GWAS meta-analysis on intracranial aneurysms consisted of two stages. The Stage 1 meta-analysis included all European ancestry individuals and consisted of individual level genotypes from 23 different cohorts, that were merged into nine European ancestry strata, based on genotyping platform and country. These strata were each analyzed in a logistic mixed model ${ }^{14}$ and then meta-analyzed, while also including summary statistics from a population-based cohort study: the Nord-Trøndelag Health Study (the HUNT Study). This resulted in 7,495 cases and 71,934 controls and 4,471,083 SNPs passing quality control (QC) thresholds (Online Methods, Supplementary Table 1). Stage 2 was a cross-ethnic metaanalysis including all Stage 1 strata and summary statistics of East Asian individuals from two population-based cohort studies: The Biobank Japan (BBJ) and the China Kadoorie Biobank (CKB). This totaled 10,754 cases and 306,882 controls and 3,527,309 SNPs in Stage 2 (Supplementary Table 1).

The Stage 1 association study resulted in 11 genome-wide significant loci (P-value $\leq$ $5 \cdot 10^{-8}$, Figure 1, Supplementary Table 2). Transethnic genetic correlation analysis showed a strong correlation between the Stage 1 meta-analysis of European ancestry and an analysis including only East Asian ancestry samples $\left(\rho_{\mathrm{g}}=0.938 \pm 0.165\right.$, standard error [SE] for genetic impact and $0.908 \pm 0.146$ for genetic effect, Supplementary Table 3). Stage 2 increased the number of genome-wide significant loci to 17 (Table 1, Figure 1). All but two loci (8q11.23, rs6997005 and 15q25.1, rs 10519203) were also associated with intracranial aneurysms in the samples of East Asian ancestry added in Stage $2(\mathrm{P}<0.05 / 11)$ and 2 loci were monomorphic in East Asians (Table 1). The Stage 2 loci included 11 novel risk loci and six previously reported risk loci ${ }^{11}$. We used conditional and joint (COJO, GCTA v1.91.1 beta) ${ }^{15}$ analysis to condition the Stage 1 GWAS summary statistics on the lead SNP in each locus. We found that none of the loci consisted of multiple independent SNPs and that each locus tagged a single causal variant (data not shown). Genomic inflation factors (lambdaGC) were 1.050 for the Stage 1 meta-analysis and 1.065 for Stage 2 (Supplementary Figure 1, Supplementary Table 4). The linkage disequilibrium score regression (LDSR) intercept was $0.957 \pm 0.008$ (SE) for the Stage 1 meta-analysis and $0.982 \pm 0.008$ for the East Asian subset. This indicated that in all GWAS analyses, observed inflation was due to polygenic architecture.

Conditioning the Stage 1 GWAS summary statistics on GWAS summary statistics for systolic and diastolic blood pressure (BP, Neale lab summary statistics [http://www.nealelab.is/blog/2017/7/19/rapid-gwas-of-thousands-of-phenotypesfor-337000-samples-in-the-uk-biobank]) using multi-trait conditional and joint (mtCOJO) ${ }^{16}$ analysis resulted in one additional genome-wide significant locus (rs2616406, $\mathrm{P}=6.221 \cdot 10^{-8}$ in the Stage 1 GWAS, $\mathrm{P}=4.499 \cdot 10^{-9}$ after mtCOJO with $\mathrm{BP}$ ). mtCOJO with smoking packyears summary statistics, or including genetic risk scores (GRSs) for smoking (cigarettes per day) ${ }^{17}$ or blood pressure related traits ${ }^{18}$ did not result in additional loci (data not shown). 


\section{Characterization of GWAS loci}

An overview of the genic position, alleles, effect size and P-value of the strongest association per locus is shown in Table 1. We used summary statistics-based Mendelian randomization (SMR), co-localization analysis using eCAVIAR, and transcriptomewide association study (TWAS, http://gusevlab.org/projects/fusion/) to annotate potential causative genes in these loci (Supplementary Tables 5-9, Supplementary Figure 2). A description of this annotation process is described in the Supplementary Note. Since SMR, eCAVIAR and TWAS all require LD reference panels, we limited the annotation to the loci identified in the European ancestry Stage 1 GWAS meta-analysis. This resulted in 11 potential causative genes on six unique loci: SLC22A5/SLC22A4/P4HA2(chr5), NT5C2/MARCKSL1P1 (chr10), FGD6/NR2C1 (chr12), PSMA4 (chr15) and BCAR1I RP11-252K23.2 (chr16) (Table 1, Supplementary Table 5). Although we did not find evidence for involvement of $S O X 17$ in the chr8 locus, previous studies did find functional evidence for $\operatorname{SOX} 17^{19,20}$. Therefore, we annotated the chr8 locus as SOX17.

In the Stage 2 GWAS, six additional loci were identified: 6q16.1, 10q23.33, 11p15.5, 12p12.2, 12q21.22, and 20p11.23. Due to the combined European and East Asian LD structures, these loci cannot reliably be mapped to genes using the above-mentioned techniques. Of the six additional loci, four have previously been linked to blood pressure, namely 6q16.1 (rs11153071) ${ }^{21}, 10 \mathrm{q} 23.33$ (rs11187838) ${ }^{22}$, rs11044991 (12p12.2) ${ }^{23}$, and rs $2681492(12 \mathrm{q} 21.22)^{23,24}$. A detailed description of the genes and loci is found in the Supplementary Note.

The product of the potentially causative gene $F G D 6^{25}$ plays a role in angiogenesis and defects may lead to a compromised formation of blood vessels. FGD6 is a vascular endothelial cell (vEC) signaling gene, involved in stress signaling in $\mathrm{vECs}^{26}$. Loss-offunction mutations in THSD1 and SOX17lead to subarachnoid hemorrhage in animal models. Products of these genes both have key roles in vECs $7,19,27 . B C A R 1$ is a ubiquitously expressed gene which protein product is a sensor for mechanical stress ${ }^{28}$. The PSMA4 locus is known for associations with a number of smoking and respiratory system traits ${ }^{29-32}$.

\section{Predictors of intracranial aneurysm rupture}

We assessed whether genetic risk factors differed between ruptured and unruptured intracranial aneurysms, using stratified GWAS analysis. The number of cases with unruptured intracranial aneurysm was small $(\mathrm{N}=2070)$. Therefore, in addition to performing a stratified GWAS on patients with a ruptured aneurysm versus patients with an unruptured intracranial aneurysm (aSAH-vs-uIA), we also performed a stratified GWAS on only patients with ruptured intracranial aneurysm versus controls (aSAH-only) and a stratified GWAS on only patients with an unruptured intracranial aneurysm versus controls (uIA-only) (Supplementary Table 4, Supplementary Figure 1e-j). Overall, 69\% of intracranial aneurysm cases had a ruptured intracranial aneurysm and $28 \%$ an unruptured intracranial aneurysm while $3.8 \%$ had an unknown rupture status. The aSAH-only and uIA-only GWASs identified a number of genome-wide significant loci, all of which reached genome-wide significance in the Stage 1 and 2 GWAS meta-analyses on intracranial aneurysms. In the aSAH-vs-ulA 
GWAS, we found no genome-wide significant loci. Furthermore, genetic correlation analysis showed a high correlation of $0.970 \pm 0.133$ (SE) between ruptured and unruptured intracranial aneurysms (Supplementary Table 3). Together these findings indicate a strong similarity in genetic architecture between ruptured and unruptured intracranial aneurysm.

\section{SNP-based heritability}

We estimated the SNP-based heritability of intracranial aneurysms to be $21.6 \pm 2.8 \%$ (SE) on the liability scale with LDSR (tool named LDSC ${ }^{33}$, https://github.com/bulik/ldsc) and 29.9 $5.4 \%$ using SumHer ${ }^{34}$ (http://dougspeed.com/sumher/, Table 2). This corresponds to an explained fraction of the twin-based heritability $\left(\mathrm{h}^{2}=41 \%{ }^{3}\right)$ of $53-73 \%$ depending on the method used (LDSC or SumHer). We used a life-time risk for unruptured intracranial aneurysms of $3 \%{ }^{1}$ for the conversion to the liability scale. Since this GWAS was an admixture of patients with ruptured and unruptured intracranial aneurysms, this prevalence may not be representative of the whole study population. Therefore, we calculated liability scale heritability using a range of life-time risk values (Supplementary Figure 3a). This shows that also when using lower life-time risk estimates $(\mathrm{K})$, the explained SNP-based heritability is substantial $\left(\mathrm{K}=0.02: \mathrm{h}^{2}=19.3 \pm 2.5 \%\right.$ [LDSC], $26.8 \pm 4.8 \%$ [SumHer]; $\mathrm{K}=0.01$ : $16.3 \pm 2.1 \%$ [LDSC], $22.6 \pm 4.1 \%$ [SumHer]).

A substantial SNP-based heritability is also found for ruptured intracranial aneurysms (SAH-only, $\mathrm{h}^{2}=0.140 \pm 0.020$ ) and unruptured intracranial aneurysms (uIAonly, $\mathrm{h}^{2}=0.223 \pm 0.044$ ). The difference between the heritability estimates could suggest differences in genetic architecture, but estimates depend on the prevalence estimate (Supplementary Figure 3b-c), meaning these differences should be interpreted with caution.

\section{Enrichment of genomic regions}

To understand the disease mechanisms of intracranial aneurysms, we applied several heritability enrichment analyses using LD-score regression (LDSR). Partitioning on functional genomic elements showed a clear enrichment of heritability in regulatory elements, including enhancer and promoter histone marks H3K4me1, H3K27Ac and H3K9Ac, super enhancers, and DNAse I hypersensitivity sites (Figure 2a). Such enrichment of regulatory elements in the genome is also seen in other polygenic traits and indicates that the architecture of intracranial aneurysms is also polygenic ${ }^{35}$. Partitioning heritability per chromosome further supported a polygenic architecture as heritability was associated with the number of SNPs on a chromosome (Figure 2b).

Tissue-specific LDSR did not show enrichment for any tissue (Supplementary Tables 10 and 11). We then performed cell-type enrichment analysis using single-cell RNA-sequencing (scRNAseq) reference data derived from mouse brain ${ }^{36}$. No enrichment was found using a scRNAseq dataset of mouse brain blood vessels ${ }^{37}$ (Supplementary Table 12). Using a larger dataset defining cell-types in the mouse brain ${ }^{36}$, we found enrichment in 'endothelial mural cells', which is a combined set of vascular endothelial and mural cells (enrichment $=2.31 \pm 0.41[\mathrm{SD}], \mathrm{P}=1.65 \cdot 10^{-3}$, Figure $2 \mathrm{c}$ ), and in midbrain neurons (enrichment $=2.23 \pm 0.37, \mathrm{P}=6.56 \cdot 10^{-4}$ ). 
LD-pruned enrichment analysis using GARFIELD showed that genes specific for blood vessels were enriched (Figure 2d, Supplementary Table 13), further supporting the role of promoters and enhancers (Figure 2e).

\section{Causal genetic roles of blood pressure and smoking}

To assess which phenotypes causally influence the risk of intracranial aneurysms, we performed generalized summary statistics-based Mendelian randomization (GSMR) using summary statistics for all phenotypes available in the UK Biobank (Supplementary Table 14). We used the Stage 1 summary statistics excluding the UK Biobank data as outcome. In this analysis, we chose a stringent value for the multiple testing threshold of 376, which was the number of traits passing the GSMR quality control parameters. Sixteen traits were statistically significant after correction for multiple testing (Figure 3a). All statistically significant traits were related to either smoking or blood pressure (BP), which are the two main clinical risk factors for unruptured intracranial aneurysms and aSAH ${ }^{1,38,39}$. To determine whether genetic predisposition for smoking and BP were causal genetic risk factors independent of one another, we conditioned the Stage 1 GWAS summary statistics on GWAS summary statistics for smoking and BP using multi-trait conditional and joint analysis (mtCOJO). We used summary statistics for both systolic BP (SBP) and diastolic BP (DBP) combined to condition on BP and summary statistics for pack-years to condition on smoking (Figure 3a, Supplementary Table 14). All GSMR effects diminished after conditioning on either BP or pack-years, and remained when conditioning on the other risk factor. The mtCOJO method itself did not affect the effect size estimates as conditioning on standing height did not affect the estimates. These findings provide strong evidence that the genetic predisposition for BP and smoking are independent genetic causes of intracranial aneurysms (Figure 3b).

Since the phenotype values of the exposure traits were inverse rank-normalized, the GSMR effect size of SBP $\left(\beta_{\mathrm{xy}}=1.058 \pm 0.187\right)$ and pack-years $\left(\beta_{\mathrm{xy}}=0.973 \pm 0.236\right)$ cannot easily be interpreted. Therefore, we performed an additional GSMR analysis for BP with an updated version of the UK Biobank GWAS (http://www.nealelab.is/uk-biobank/), including raw phenotype values for quantitative traits (Supplementary Table 15). For BP traits, the GSMR analysis resulted in an effect size estimate of $0.095 \pm 0.019$ for DBP and $0.047 \pm$ 0.011 for SBP, meaning an $8-12 \%$ increase in intracranial aneurysm risk per mmHg increase of DBP and a 3.7-6\% increase in intracranial aneurysm risk per mmHg increase of SBP, assuming a linear effect of $\mathrm{BP}$ on intracranial aneurysm liability. In addition, age at high BP diagnosis had a significant GSMR effect $\left(\mathrm{P}=1.79 \cdot 10^{-4}, \beta_{\mathrm{xy}}=0.163 \pm 0.044\right)$, indicating an increase in intracranial aneurysm risk of $13-23 \%$ for each year of additional high BP exposure. We did not include smoking quantitative traits, because these were not normally distributed (data not shown) and could, therefore, lead to a biased effect estimate.

We then tested whether the effects of smoking and BP were different between ruptured (SAH-only) and unruptured intracranial aneurysms (uIA-only, Supplementary Table 16). The GSMR effect sizes followed the same trend for all phenotypes, but 'Hypertension (Self-reported)' had a stronger effect on ruptured intracranial aneurysms (SAH-only: $b_{x y}=6.74 \pm 0.61[S E]$, all intracranial aneurysms: $2.97 \pm 0.42$, uIA-only: $2.38 \pm 0.70$ ), while 
amlodipine use had a weaker effect on unruptured intracranial aneurysms and became statistically non-significant (uIA-only: $b_{x y}=4.77 \pm 3.90, P=0.22$, all intracranial aneurysms: $\mathrm{b}_{\mathrm{xy}}=11.4 \pm 2.10, \mathrm{P}=5.25 \cdot 10^{-8}$, SAH-only: $\left.\mathrm{b}_{\mathrm{xy}}=13.1 \pm 2.60, \mathrm{P}=5.25 \cdot 10^{-7}\right)$. Although the effect of self-reported hypertension on SAH-only was stronger, conditioning on blood pressure using mtCOJO mitigated the effect $\left(b_{x y}=1.02 \pm 0.45, P=0.024\right.$, data not shown). Since the power to detect GSMR effects in the UIA-only sample is much lower compared to all intracranial aneurysms and SAH-only due to limited sample size, further investigation is required to make inferences about genetic risk factors for rupture.

Traits influencing female hormones are suggested to play a role in aSAH risk ${ }^{40}$. Only two female hormone-related traits had enough genome-wide significant risk loci to pass GSMR quality control. These were 'age when periods started (menarche)' and 'had menopause'. Neither of these showed a causal relationship with intracranial aneurysms in the GSMR analysis (Supplementary Table 14).

\section{Drivers of genetic correlation with vascular traits}

To identify traits correlated with intracranial aneurysms, we analyzed Stage 1 summary statistics using LDHub ${ }^{41}$. LDHub includes a subset of the summary statistics used for GSMR and a number of summary statistics from publicly available sources. Traits that showed correlations that reached the Bonferroni threshold for multiple testing $(\mathrm{p}=0.05 / 464)$ included several blood pressure (BP)-related traits, including diastolic BP (DBP) $\left(\rho_{\mathrm{g}}=0.223\right.$, $\left.\mathrm{P}=5.40 \cdot 10^{-9}\right)$ and systolic BP (SBP) $\left(\rho_{\mathrm{g}}=0.256, \mathrm{P}=1.34 \cdot 10^{-8}\right)$ and smoking traits, such as pack-years $\left(\rho_{\mathrm{g}}=0.330, \mathrm{P}=7.87 \cdot 10^{-8}\right)$ (Supplementary Table 17$)$.

We used LDSR to calculate the genetic correlation of intracranial aneurysms with other stroke subtypes - ischemic stroke (IS) ${ }^{42}$ and intracerebral hemorrhage (ICH) -, with other vascular malformation types - intracranial arteriovenous malformation (AVM) ${ }^{43}$ and cervical artery dissection ${ }^{44}$-, and with abdominal aortic aneurysm (AAA) ${ }^{45}$. For IS, a correlation of $0.195 \pm 0.079(\mathrm{P}=0.014)$ was found with intracranial aneurysms (Figure 3c, Supplementary Table 3). After conditioning the intracranial aneurysm GWAS on either BP or on pack-years, which are clinical risk factors for both IS and intracranial aneurysms $1,38,39,46$, the correlation was no longer statistically significant and reduced to $0.121 \pm 0.081$ for BP and $0.147 \pm 0.084$ for pack-years. The correlation disappeared after conditioning on both risk factors $\left(\rho_{\mathrm{g}}=0.009 \pm 0.083, \mathrm{P}=0.916\right)$. When conditioning on an unrelated but heritable trait (standing height), the correlation remained $\left(\rho_{\mathrm{g}}=0.238 \pm 0.081\right.$, $\mathrm{P}=0.003)$. No genetic correlation was found for any of the IS subtypes.

We found a statistically significant genetic correlation between intracranial aneurysms and ICH $\left(\rho_{\mathrm{g}}=0.447 \pm 0.184, \mathrm{P}=0.015\right)$, which was mainly driven by deep ICH $\left(\rho_{\mathrm{g}}=0.516 \pm 0.198\right.$, $\mathrm{P}=0.009)$, and not by lobar ICH $(\mathrm{P}=0.534)$. After conditioning the intracranial aneurysm GWAS on either BP or pack-years, which are also important risk factors for $\mathrm{ICH}^{47}$, the correlation with deep ICH decreased $\left(\rho_{\mathrm{g}}=0.288 \pm 0.189\right.$ for BP and $0.234 \pm 0.192$ for pack-years) and was no longer statistically significant. Conditioning on height had a much smaller effect $\left(\rho_{\mathrm{g}}=0.380 \pm 0.196\right)$. 
A genetic correlation was found between intracranial aneurysms and AAA $\left(\rho_{\mathrm{g}}=0.302 \pm 0.105, \mathrm{P}=0.004\right)$. Conditioning on pack-years strongly reduced the correlation between intracranial aneurysms and AAA $\left(\rho_{\mathrm{g}}=0.173 \pm 0.117, \mathrm{P}=0.138\right)$, whereas BP did not $\left(\rho_{\mathrm{g}}=0.264 \pm 0.117, \mathrm{P}=0.024\right)$.

There was no genetic correlation between intracranial aneurysms and carotid artery dissection $\left(\rho_{\mathrm{g}}=0.151 \pm 0.180, \mathrm{P}=0.401\right)$; whereas for vertebral artery dissection and the combined set of vertebral and carotid artery dissection, a larger, albeit non-statistically significant, estimate was observed $\left(\rho_{\mathrm{g}}=0.281 \pm 0.159, \mathrm{P}=0.077\right.$ and $\rho_{\mathrm{g}}=0.174 \pm 0.149$, $\mathrm{P}=0.066$, respectively) (Supplementary Table 3 ). For AVM, a negative SNP-based heritability was estimated, which could be due to the small sample size of this GWAS $(1,123$ cases and 1,935 controls). Therefore, we performed a lookup of all SNPs identified in the Stage 1 and 2 intracranial aneurysm GWAS in the summary statistics of the AVM GWAS 43 but were unable to replicate any of these SNP associations $(\mathrm{P}<0.05 / 17)$ (Supplementary Table 18).

\section{Drug target enrichment}

To identify pleotropic pathways between intracranial aneurysms and other diseases that contain known drug targets, we assessed enrichment in genes targeted by drugs and drug classes ${ }^{48}$. Gene-based P-values were calculated with MAGMA, resulting in 29 genes that passed the Bonferroni threshold for multiple testing $(\mathrm{P}<0.05 / 18106$, Supplementary Table 19). The anti-hypertensive drugs ambrisentan and macitentan showed a statistically significant enrichment $\left(\mathrm{P}=1.35 \cdot 10^{-5}\right.$, Supplementary Table 20$)$ which was driven by a single gene $(E D N R A)$. Drug class enrichment analysis showed that drugs in the classes 'antiepileptics' were enriched (area under the curve $[\mathrm{AUC}]=0.675, \mathrm{P}=8 \cdot 10^{-5}$, Supplementary Table 21). The most statistically significant enriched drugs within this class are blockers of $\mathrm{Na}^{+}$and $\mathrm{Ca}^{2+}$ channels, namely phenytoin, zonisamide and topiramate ${ }^{49}$ (Supplementary Table 20). These channels are important in blood pressure regulation, as well as in several other biological mechanisms. The other enriched drug class is 'sex hormones + modulators of the genital system' ( $\mathrm{AUC}=0.652, \mathrm{P}=2.02 \cdot 10^{-4}$ ). We also used MAGMA to study enrichment in gene pathways, but found no statistically significant results (Supplementary Table 22).

\section{Discussion}

We identified 11 novel risk loci for intracranial aneurysms and confirmed six previously identified risk loci, making a total of 17 risk loci for intracranial aneurysms. A SNP-based heritability of $21.6 \%$ was found, explaining over half of the total heritability. We showed strong evidence that the majority of intracranial aneurysm heritability is polygenic. Our results further highlight several major features of the genetic architecture of intracranial aneurysms. First, we identified endothelial cells as a key cell type in intracranial aneurysm risk. Second, we showed that, out of 375 tested traits, smoking and BP predisposition were the main genetic risk factors for intracranial aneurysms. Third, we showed that the main drivers of the genetic correlation between intracranial aneurysms and other stroke types and between intracranial aneurysms and abdominal aortic aneurysms are genetic predisposition 
for smoking and blood pressure. Last, we found pleiotropic characteristics of anti-epileptic drugs and sex hormones with intracranial aneurysms.

Through gene-mapping incorporating gene expression datasets and distinct bioinformatics analyses, we were able to identify 11 potential causative genes within 6 of the Stage 1 risk loci. Many of these genes have known or putative roles in blood vessel function and blood pressure regulation. We found heritability enrichment in genes that are specifically expressed in a combined set of endothelial and mural cells, and not in other vascular cell types. Together, the identified potential causative genes and heritability enrichment analyses suggest an important role of the vascular endothelial cell (vEC) in intracranial aneurysm development and rupture.

Through genetic correlation and formal causal inference methods, we established that genetic predisposition for smoking and BP are the most important independent genetic risk factors for intracranial aneurysms ${ }^{1}$. First, using causal inference with GSMR, we showed that genetic predisposition for these traits drives a causal increase in intracranial aneurysm risk. Then, using multi-trait conditional analysis, we showed that smoking and high BP are causative of intracranial aneurysms, independent of one another. By using non-transformed continuous systolic blood pressure (SBP) and diastolic blood pressure (DBP) measures in the UK Biobank, we estimated the increase in intracranial aneurysm risk per $1 \mathrm{mmHg}$ increase of SBP to be 3.7-6\%, and that of DBP to be $8-12 \%$. These strong effects provide genetic evidence for clinical prevention by lowering blood pressure. Since smoking dose is not normally distributed, we were not able to estimate a quantitative effect of smoking on intracranial aneurysms, but this has been done before using non-genetic methods ${ }^{50-52}$. Future studies that model risk prediction using polygenic risk scores should determine whether the polygenic risks of genetic risk factors for intracranial aneurysms are clinically relevant risk factors for the disease.

We found that genetic correlations of intracranial aneurysms with ischemic stroke (IS) and deep intracerebral hemorrhage (ICH) are mainly driven by genetic predisposition for smoking and BP. For ICH, conditioning on smoking and BP did not completely mitigate the genetic correlation with intracranial aneurysms, suggesting additional shared genetic causes. For vertebral artery dissection, a substantial, but not statistically significant correlation with intracranial aneurysms was found, whereas this was absent in carotid artery dissection. We showed that the genetic correlation between intracranial aneurysms and AAA was driven by smoking, but not by BP. This implies that intracranial aneurysms are more dependent on BP compared to AAA. This observation could be a result of different ratios of unruptured and ruptured aneurysms included in the two GWASs. The AAA GWAS consists of mainly unruptured $\mathrm{AAA}^{45}$, and while the role of $\mathrm{BP}$ on AAA rupture is clear, the effect on developing AAA is a matter of debate ${ }^{53}$.

One of the main aims of intracranial aneurysm research is to prevent rupture of intracranial aneurysms and thus avoid the devastating consequences of aSAH. We performed various analyses in an attempt to identify genetic predictors specific for intracranial aneurysm rupture. Instead, we found a very strong genetic correlation between ruptured and 
unruptured intracranial aneurysms. These analyses together indicate that the common variant genetic architecture of ruptured and unruptured aneurysms are strikingly similar.

The heritability of unruptured intracranial aneurysms has never been studied in twins, and may, therefore, not be an optimal estimate for intracranial aneurysm heritability. One twin study estimated the heritability of aSAH at $41 \%^{3}$. Our finding that the genetic architecture of uIA and aSAH are similar suggests that this heritability estimate may also be accurate for unruptured intracranial aneurysms. This means that in European ancestry populations, 53 to $73 \%$ of the heritability of intracranial aneurysms can be explained by variants tagged in this GWAS.

Using transethnic genetic correlation, we found a remarkable similarity of genetic architecture between the European ancestry and East Asian ancestry GWASs of more than $90.8 \pm 14.6 \%$ (SE). This indicates that the majority of common-variant genetic causes are the same, regardless of ancestry. However, since the LD structures remain distinct, current methods for summary statistic-based enrichment analysis cannot effectively account for population-specific variation in a cross-ethnic GWAS.

Drug class enrichment showed pleiotropic characteristics of anti-epileptic drugs and sex hormones with the genetic association of intracranial aneurysms. It has been suggested that sex hormones might play a role in intracranial aneurysms ${ }^{40}$, potentially explaining why women have a higher intracranial aneurysm risk than men ${ }^{1}$. However, as causal inference analysis with GSMR did not show evidence for the involvement of female hormones, further investigation is required. Enrichment of the anti-epileptic drug class may indicate shared disease mechanisms between intracranial aneurysms and epilepsy. The main mechanism of anti-epileptic drugs is through blocking $\mathrm{Na}^{+}$and $\mathrm{Ca}^{2+}$ ion-channels ${ }^{49}$. Together with other ion channels, these play essential roles in contraction and relaxation of the blood vessels ${ }^{54}$. Mutations in the ion-channel gene PKD2 (TRRP2) are known to cause intracranial aneurysms. This gene product, along with other members of the TRP gene family, regulates systemic blood pressure through vasoconstriction and vasodilation ${ }^{55,56}$. More research on the effect of anti-epileptics on vascular tension and blood pressure will enhance our understanding of the disease-causing mechanisms. Furthermore, this could help to identify methods of intracranial aneurysm prevention using anti-epileptics or related drugs.

In conclusion, we performed a GWAS meta-analysis on intracranial aneurysms identifying 11 new risk loci, confirming 6 previously identified risk loci and explaining over half of the heritability of intracranial aneurysms. We found strong evidence for a polygenic architecture. Through gene-mapping and heritability enrichment methods, we discovered a possible role for endothelial cells in intracranial aneurysm development. We showed that the genetic architecture of unruptured and ruptured aneurysms are very similar. The well-known clinical risk factors, smoking and hypertension, were identified as main genetic drivers of intracranial aneurysms. These risk factors also explained most of the similarity to other stroke types, IS and deep ICH, which could open a window for clinical prevention. We also found pleiotropic effects between intracranial aneurysms and anti-epileptic drugs, which require further investigation to understand the shared mechanisms of intracranial aneurysms 
and epilepsy. Our findings represent a major advance in understanding the pathogenesis of intracranial aneurysms and a significant step towards the development of effective genetic risk prediction and prevention of intracranial aneurysm development and subsequent aSAH in the future.

\section{Online Methods}

\section{Recruitment and diagnosis}

Detailed cohort descriptions are given in the Supplementary Note. In brief, all intracranial aneurysm cases have a saccular intracranial aneurysm. We included both cases with ruptured-thus with aSAH- and unruptured intracranial aneurysms confirmed using imaging. Patients with conditions known to predispose to intracranial aneurysms, including autosomal dominant polycystic kidney disease, Ehlers-Danlos disease and Marfan's syndrome, were excluded. All controls were unselected controls. Controls were matched by genotyping platform and country on cohort-level.

\section{Genotype data quality control}

Cohorts for which individual level data were available are specified in Supplementary Table 1. An overview of inclusion and exclusion criteria, data collection and genotyping methods for each cohort are given in the Supplementary Note. Genotypes were lifted to reference genome build GRCh37. An extensive QC was performed on each cohort, described in detail in the Supplementary Note. Cohorts were merged into strata based on genotyping platform and country. An overview of strata compositions is given in Supplementary Table 1. Next, QC was performed on each stratum, outlined in the Supplementary Note. Genotypes were imputed against the Haplotype Reference Consortium (HRC) release 1.1. After imputation, another set of QC steps was taken, which is described in the Supplementary Note. An overview of the number of SNPs, cases and controls excluded in the QC is shown in Supplementary Table 1.

\section{Individual level association analysis}

For each stratum, single-SNP associations were calculated using SAIGE (0.29.3) ${ }^{14}$. SAIGE uses a logistic mixed model to account for population stratification and saddle point approximation to accurately determine P-values even in the presence of case-control imbalance. Details on how these steps were performed are described in the Supplementary Note.

\section{Meta-analysis}

We meta-analyzed association statistics from our individual level SAIGE analysis with association statistics prepared by other groups who used the same analysis pipeline. There were two meta-analysis stages: Stage 1, including all individual level data and the European ancestry summary statistics (HUNT Study), and Stage 2 including all individual level data and all summary statistics (HUNT Study, China Kadoorie Biobank, Biobank Japan). Summary statistics that were generated by other groups were cleaned prior to meta-analysis, as described in the Supplementary Note. We used METAL (release 2011-03-25) ${ }^{57}$ for the 
inverse-variance weighted meta-analysis across all studies. Only SNPs present in at least $80 \%$ of the strata were included.

\section{Conditional analysis}

To investigate whether a genome-wide significant locus consisted of multiple independent signals we used GCTA-COJO. ${ }^{15}$ COJO uses GWAS summary statistics and the LD structure of a reference panel to iteratively condition GWAS summary statistics on top SNPs. We used control samples from stratum sNL2 (Doetinchem Cohort Study) as a reference panel for LD estimation. We used a stepwise approach to condition on the top independent SNPs with $\mathrm{P}<5 \cdot 10^{-8}$ and minor allele frequency (MAF) $>0.01$. In addition, we conditioned the summary statistics on the identified top independent hits to determine if any additional signal remained.

\section{Genetic risk score analysis}

To investigate the effect of genetic risk for blood pressure (BP) and smoking on intracranial aneurysms, we used its genetic risk scores (GRS) as covariates in a SAIGE association model. Summary statistics for BP-related traits ${ }^{18}$ and cigarettes per day (CPD) ${ }^{17}$ were obtained. SNPs to include in the GRS models were determined using different LD thresholds by clumping ( $\mathrm{r}^{2}$ of $0.1,0.2,0.5,0.8$ or 0.9$)$. Individual level GRS were calculated using plink v1.9 (https://www.cog-genomics.org/plink2/). The optimal models were selected based on the highest fraction of variance explained (adj.r.squared from $\operatorname{lm}()$ in R/3.6.1). An optimal $\mathrm{r}^{2}$ of 0.1 and 0.9 were selected for BP and CPD, respectively. A set of 20,000 individuals from the UK Biobank, including all intracranial aneurysm cases, was used to train the model. Individual levels GRSs using the optimized set of SNPs was used as a covariate in an association analysis using SAIGE.

\section{eQTL-based gene mapping}

We used eCAVIAR ${ }^{58}$ to determine colocalization of GWAS hits with eQTLs. Vascular and whole blood eQTLs from GTEx v7 were used. eCAVIAR used SNP Z-scores and LD correlation values to calculate a colocalization posterior probability (CLPP) of a trait GWAS locus and an eQTL. eCAVIAR requires an LD matrix to determine colocalization of eQTLs and GWAS hits. We calculated LD in SNPs 1MB on both sides of the SNPs with lowest Stage 1 GWAS P-value, using European ancestry Health and Retirement Study (HRS dbGaP accession code phs000428.v2.p2) samples as a reference. Multiple causal SNPs were allowed.

TWAS is a method to perform differential expression analysis with eQTL-based predicted transcript levels. We used a summary statistics-based approach integrated in FUSION ${ }^{59}$. We used the 1000 Genomes LD weights provided by FUSION, and vascular and blood eQTL datasets provided on the FUSION reference webpage (http://gusevlab.org/projects/ fusion/). Default settings were used for all other options.

SMR ${ }^{60}$ was used to highlight genes the expression of which has a causal influence on intracranial aneurysm risk. eQTL reference datasets from vascular tissues and blood provided by the creators of SMR were used. These include: CAGE, GTEx V7 (aorta, 
coronary artery, tibial artery and whole blood) and Westra (https://cnsgenomics.com/ software/smr/\#DataResource). eQTLs with a p-value below $5 \cdot 10^{-8}$ were selected. The MAF cutoff was set at 0.01 . European ancestry samples from the HRS were used as LD reference panel. Both the single SNP and multi-SNP approaches were used.

eCAVIAR, TWAS and SMR results were used to annotate genes to genome-wide significant GWAS loci identified in the Stage 1 GWAS meta-analysis. This approach is explained in more detail in the Supplementary Note.

\section{SNP-based heritability}

To calculate SNP-based heritability, we used LDSC (1.0.0) ${ }^{33}$ to perform LD-score regression (LDSR), and we used SumHer ${ }^{34}$. LDSC makes the assumption that the contribution of each SNP to the total SNP heritability is normally distributed and not affected by MAF or LD. SumHer is the summary statistics based equivalent of an LDadjusted kinship (LDAK) method to estimate SNP heritability and, instead, assumes that heritability is higher for low MAF variants and lower in high LD regions. In addition, SumHer models inflation due to residual confounding as a multiplicative parameter, whereas LDSC models this additively (the LDSR intercept). Heritability estimates were converted to the liability scale using effective sample size. More details and the rationale of these analyses are described in the Supplementary Note.

\section{Functional enrichment analysis using LDSC}

To assess enrichment of heritability in functional annotations, tissues, chromosomes and minor allele frequency (MAF) bins, we used stratified LD-score regression with LDSC $^{61}$. When available we used the publicly available partitioned LD scores for pre-defined annotations provided by the LDSC authors (https://data.broadinstitute.org/ alkesgroup/LDSCORE/), otherwise we calculated our own LD scores using European ancestry samples from the 1000 Genomes (1000G) project. To further assess cell typespecific enrichment, we used a method introduced by Skene et al ${ }^{36}$. For this analysis, we used single-cell RNA sequencing (scRNAseq) gene expression data derived from mouse brain to define gene sets specific to cell types in brain ${ }^{36}$ and brain blood vessels ${ }^{37}$. A detailed description of the rationale and parameters is given in the Supplementary Note.

\section{Functional enrichment analysis using GARFIELD}

The GWAS functional enrichment tool GARFIELD v2 ${ }^{62}$ was used to explore regulatory, functional and tissue-specific enrichment of the GWAS summary statistics. It determines whether GWAS SNPs reaching a certain P-value threshold are enriched in annotations of interest compared to the rest of the genome while accounting for distance to nearest transcription start site, MAF and LD. We used the default annotations provided by the authors to test enrichment in tissues (https://www.ebi.ac.uk/birney-srv/GARFIELD/). We tested enrichment of SNPs passing P-value thresholds for every $\log _{10}$-unit between 0.1 and $10^{-8}$. A more detailed description of the method is given in the Supplementary Note. 


\section{Genetic correlation}

We assessed correlation between intracranial aneurysms and other traits using LDHub and LD-score regression (LDSR) with LDSC. To assess genetic correlation between intracranial aneurysms and many non-stroke-related traits, we used LD Hub ${ }^{41}$. This platform uses LDSR to assess genetic correlation with a large number of publicly available GWASs. For the correlation of intracranial aneurysms and other stroke subtypes, we obtained summary statistics for All Stroke (AS), Cardioembolic Stroke (CE), Any Ischemic Stroke (AnyIS), Large Artery Stroke (LAS), Small Vessel Disease (SVD) ${ }^{42}$, Deep, Lobar, and combined Intracerebral Hemorrhage (ICH) ${ }^{63}$, carotid- and vertebral artery dissection ${ }^{44}$, Arteriovenous Malformation (AVM) ${ }^{43}$ and Abdominal Aortic Aneurysms (AAA) ${ }^{45}$. We used LDSC to calculate genetic correlation. LD scores from European ancestry individuals from $1000 \mathrm{G}$ were calculated for SNPs in the HapMap 3 SNP set and used to calculate genetic correlation. Since the heritability estimate was negative for AVM, due to the small sample size, we performed a SNP lookup of the Stage 2 intracranial aneurysm loci that passed the multiple testing threshold $\left(\mathrm{P}<5 \cdot 10^{-8}\right)$ from the GWAS of $\mathrm{AVM}^{43}$.

\section{Conditional genetic correlation}

We used mtCOJO ${ }^{16}$ to condition Stage 1 intracranial aneurysm GWAS summary statistics on summary statistics from the Neale lab UK Biobank GWAS release 1 (http://www.nealelab.is/blog/2017/7/19/rapid-gwas-of-thousands-of-phenotypesfor-337000-samples-in-the-uk-biobank) for smoking and blood pressure (BP) following a method described previously ${ }^{16}$. The resulting summary statistics were then used to calculate genetic correlation between intracranial aneurysms, conditioned on another trait, and other vascular diseases. LD scores supplied by LDSC (eur_W_ld_chr/[chr].12.Idscore.gz) were used. European ancestry control samples from stratum sNL2 (from the Doetinchem Cohort Study) were used as an LD reference panel. All other settings were left as default.

\section{Trans-ancestry genetic correlation}

Popcorn version 0.9.9 ${ }^{64}$ was used to assess genetic correlation between intracranial aneurysm cohorts of European and East Asian ancestry. Popcorn uses separate LD score reference panels per ancestry to account for differences in LD structure between cohorts. We used LD scores provided by the authors of the Popcorn tool (https://github.com/brielin/ Popcorn) for European and East Asian descent (EUR_EAS_all_gen_[eff/imp].cscore). We calculated the genetic correlation for both genetic impact and genetic effect.

\section{Mendelian randomization}

To infer causal genetic effects of exposure traits on intracranial aneurysms (the outcome), we used GSMR ${ }^{16}$. We used a meta-analysis of all European ancestry strata, except the UK biobank (stratum sUK2), as outcome. As exposures we used summary statistics of 2419 traits analyzed using UK Biobank data, prepared by the Neale lab, release 2017 (http://www.nealelab.is/blog/2017/7/19/rapid-gwas-of-thousands-ofphenotypes-for-337000-samples-in-the-uk-biobank). For a second GSMR run with raw quantitative phenotypes we used the 2019 GWAS release from the same group. GSMR 
was run using the GCTA wrapper (v1.92.2). More details on the method and settings are described in the Supplementary Note.

In order to determine which of the top significant GSMR traits were independent genetic causes of intracranial aneurysms, the Stage 1 GWAS summary statistics were conditioned on the top traits, i.e. smoking and blood pressure (BP). Conditioning was done using mtCOJO (GCTA v1.92.2 beta) as described in the Conditional genetic correlation section of the Online Methods.

\section{Drug target enrichment}

Drug target enrichment analysis was performed according to a previously described method $^{48}$. Gene-wise P-values were calculated with MAGMA v1.06 using a combined approach of average and top P-values per gene region. Gene-set analysis was performed using MAGMA, with pathways curated from MSigDB ${ }^{65,66}$, TargetValidation (https:// www.targetvalidation.org), and with drug-target sets described previously ${ }^{48}$. Drug-class enrichment analysis was performed using a Wilcoxon-Mann-Whitney test. Drug gene-set P-values were tested for enrichment in drug-classes. Enrichment was expressed as the area under the curve (AUC). AUCs were compared between drug gene-sets within a drug class and all other drug gene-sets.

\section{Statistics}

The different statistical tests used in the different analysis methods are as follows: 1 . SAIGE: Logistic mixed model with saddle-point approximation for P-values. Resulting beta values are on the logit scale. 2. METAL: Inverse-variance weighted meta-analysis. Resulting betas are on the same scale as the input (here, logit scale). 3. eCAVIAR: Directly calculates a colocalization posterior probability from expression and trait GWAS effect sizes using Bayes' rule. 4. TWAS: Uses to calculate a Z-score, which is tested against a null-distribution of mean zero and unit variance to calculate a P-value. 5. SMR: The Mendelian Randomization effect of exposure (gene expression) $\mathrm{x}$ on outcome $\mathrm{y}$ is the ratio of the estimate of the effect of SNP $z$ on outcome $y$ and SNP $z$ on exposure $x$. The SNP effect Z-scores are used to calculate a $C^{2}$-statistic with one degree of freedom. 6 . LDSC: Weighted linear regression, where weights are the inverse of the LD score of a SNP. The slope is divided by sample size and multiplied by the number of SNPs. Standard errors are obtained by jackknife method. 7. GARFIELD: Calculates enrichment odds ratios using logistic regression, accounting for $\mathrm{LD}$, distance to transcription start site, and binary annotations. 8. POPCORN: Maximum likelihood test. Standard error is calculated using a block jackknife method. 9. GSMR: Two-sided linear regression after excluding pleiotropic SNPs using 'heterogeneity in dependent instrument'-test. 10. MAGMA (gene test): Uses a multiple linear regression to calculate gene effects. Subsequent P-value is derived from two-sided F-test. MAGMA (gene set test): Drug P-values are calculated by comparing gene Z-scores (derived from P-values reported in Supplementary Table 19) in the gene set to those outside the gene set. P-values are derived from one-sided t-test. 11. SumHer: Conceptually similar to LDSC, but with different weight based on linkage disequilibrium and minor allele frequency. 


\section{Supplementary Material}

Refer to Web version on PubMed Central for supplementary material.

\section{Authors}

Mark K. Bakker ${ }^{1}{ }^{*}$, Rick A.A. van der Spek ${ }^{1}$, Wouter van Rheenen ${ }^{1}$, Sandrine Morel $^{2,3}$, Romain Bourcier ${ }^{4,5}$, Isabel C. Hostettler ${ }^{6,7}$, Varinder S. Alg ${ }^{8}$, Kristel R. van Eijk ${ }^{1}$, Masaru Koido ${ }^{9,10}$, Masato Akiyama9 ${ }^{9} 11,12$, Chikashi Terao ${ }^{9}$, Koichi Matsuda $^{13}$, Robin G. Walters ${ }^{14,15}$, Kuang Lin ${ }^{14}$, Liming Li ${ }^{16}$, Iona Y. Millwood ${ }^{14,15}$, Zhengming Chen ${ }^{14,15}$, Guy A. Rouleau ${ }^{17}$, Sirui Zhou ${ }^{18}$, Kristiina Rannikmäe ${ }^{19}$, Cathie L.M. Sudlow ${ }^{19,20}$, Henry Houlden ${ }^{21}$, Leonard H. van den Berg ${ }^{1}$, Christian Dina ${ }^{4}$, Olivier Naggara ${ }^{22,23}$, Jean-Christophe Gentric ${ }^{24}$, Eimad Shotar ${ }^{25}$, François Eugène $^{26}$, Hubert Desal ${ }^{4,5}$, Bendik $S$. Winsvold ${ }^{27,28}$, Sigrid Børte ${ }^{28,29,30}$, Marianne Bakke Johnsen $28,29,30$, Ben M. Brumpton ${ }^{28}$, Marie Søfteland Sandvei ${ }^{31,32}$, Cristen J. Willer ${ }^{33}$, Kristian Hveem ${ }^{28,34}$, John-Anker Zwart ${ }^{27,28,30}$, W. M. Monique Verschuren ${ }^{35,36}$, Christoph M. Friedrich ${ }^{37,38}$, Sven Hirsch ${ }^{39}$, Sabine Schilling ${ }^{39}$, Jérôme Dauvillier ${ }^{40}$, Olivier Martin ${ }^{40}$,

HUNT All-In Stroke41

Amy E Martinsen ${ }^{1}$, Anne Hege Aamodt ${ }^{2}$, Anne Heidi Skogholt ${ }^{3,4}$, Else Charlotte Sandset ${ }^{5,6}$, Espen S Kristoffersen ${ }^{7,8}$, Hanne Ellekjaer 9,10 , Ingrid Heuch $^{8}$, Jonas Bille Nielsen ${ }^{3,11,12}$, Knut Hagen ${ }^{13,14}$, Lars Fritsche ${ }^{15}$, Laurent F. Thomas ${ }^{3,4}$, Linda Pedersen ${ }^{8}$, Maiken E Gabrielsen ${ }^{3}$, Maria Dehli Vigeland,

Oddgeir Holmen ${ }^{16}$, Wei Zhou

China Kadoorie Biobank Collaborative Group41

Junshi Chen ${ }^{1}$, Zhengming Chen $(\mathrm{PI})^{2,3}$, Robert Clarke ${ }^{2}$, Rory Collins ${ }^{2}$, Yu Guo ${ }^{4}$, Liming Li (PI) ${ }^{5}$, Depei Liu ${ }^{4}$, Jun Lv ${ }^{5}$, Richard Peto ${ }^{2}$, Robin Walters ${ }^{2,3}$, Daniel Avery², Ruth Boxall ${ }^{2,3}$, Derrick Bennett ${ }^{2}$, Yumei Chang ${ }^{2}$, Yiping Chen ${ }^{2,3}$, Zhengming Chen ${ }^{2,3}$, Robert Clarke², Huaidong Du2,3, Wei Gan², Simon Gilbert ${ }^{2}$, Alex Hacker $^{2}$, Michael Hill2,3, Michael Holmes ${ }^{2,3}$, Andri lona², Christiana Kartsonaki ${ }^{2,3}$, Rene Kerosi ${ }^{2}$, Ling Kong ${ }^{2}$, Garry Lancaster ${ }^{2}$, Sarah Lewington ${ }^{2,3}$, Kuang Lin ${ }^{2}$, John McDonnell2, lona Millwood ${ }^{2,3}$, Qunhua Nie ${ }^{2}$, Paul Ryder $^{2}$, Sam Sansome $^{2}$, Dan Schmidt-Valle ${ }^{2}$, Paul Sherliker ${ }^{2}$, Rajani Sohoni ${ }^{2}$, Becky Stevens ${ }^{2}$, lain Turnbull ${ }^{2}$, Robin Walters ${ }^{2,3}$, Lin Wang ${ }^{2}$, Neil Wright ${ }^{2}$, Ling Yang ${ }^{2,3}$, Xiaoming Yang ${ }^{2}$, Pang $\mathrm{Yao}^{2}$, Zheng Bian ${ }^{4}$, Yu Guo ${ }^{4}$, Xiao Han ${ }^{4}$, Can Hou${ }^{4}$, Jun Lv ${ }^{5}$, Pei Pei ${ }^{4}$, Chao $\mathrm{Liu}^{4}$, Canqing $\mathrm{Yu}^{4}$, Zengchang Pang ${ }^{6}$, Ruqin $\mathrm{Gao}^{6}$, Shanpeng $\mathrm{Li}^{6}$, Shaojie Wang ${ }^{6}$, Yongmei Liu ${ }^{6}$, Ranran Du' ${ }^{6}$, Liang Cheng ${ }^{6}$, Xiaocao Tian ${ }^{6}$, Hua Zhang ${ }^{6}$, Yaoming Zhai $^{6}$, Feng Ning ${ }^{6}$, Xiaohui Sun ${ }^{6}$, Feifei Li ${ }^{6}$, Silu Lv ${ }^{7}$, Junzheng $\mathrm{Wang}^{7}$, Wei Hou ${ }^{7}$, Mingyuan Zou ${ }^{8}$, Shichun Yan ${ }^{8}$, Xue Zhou ${ }^{8}$, Bo Yu${ }^{9}$, Yanjie Li ${ }^{9}$, Qinai Xu ${ }^{9}$, Quan Kang $^{9}$, Ziyan Guo ${ }^{9}$, Dan Wang ${ }^{10}$, Ximin $\mathrm{Hu}^{10}$, Jinyan Chen ${ }^{10}$, Yan $\mathrm{Fu}^{10}$, Xiaohuan Wang $^{10}$, Min Weng ${ }^{11}$, Zhendong Guo ${ }^{11}$, Shukuan Wu${ }^{11}$, Yilei $\mathrm{Li}^{11}$, Huimei $\mathrm{Li}^{11}$, Ming $\mathrm{Wu}^{11}$, Yonglin Zhou ${ }^{12}$, Jinyi Zhou ${ }^{12}$, Ran Tao ${ }^{12}$, Jie Yang ${ }^{12}$, Jian Su ${ }^{12}$, Fang 
$\mathrm{liu}^{13}$, Jun Zhang ${ }^{13}$, Yihe $\mathrm{Hu}^{13}$, Yan $\mathrm{Lu}^{13}$, Liangcai Ma13, Aiyu Tang ${ }^{13}$, Yujie Hua ${ }^{13}$, Jianrong Jin ${ }^{14}$, Jingchao Liu ${ }^{14}$, Zhenzhu Tang ${ }^{15}$, Naying Chen ${ }^{15}$, Ying Huang ${ }^{15}$, Mingqiang Li ${ }^{16}$, Jinhuai Meng ${ }^{16}$, Rong Pan ${ }^{16}$, Qilian Jiang ${ }^{16}$, Jian Lan ${ }^{16}$, Yun Liu ${ }^{16}$, Liuping Wei ${ }^{16}$, Liyuan Zhou ${ }^{16}$, Ningyu Chen ${ }^{16}$, Ping Wang ${ }^{16}$, Fanwen Meng ${ }^{16}$, Yulu Qin Sisi Wang ${ }^{16}$, Xianping Wu ${ }^{17}$, Ningmei Zhang ${ }^{17}$, Xiaofang Chen ${ }^{17}$, Weiwei Zhou $^{17}$, Guojin Luo ${ }^{18}$, Jianguo Li ${ }^{18}$, Xiaofang Chen ${ }^{18}$, Xunfu Zhong ${ }^{18}$, Jiaqiu Liu ${ }^{18}$, Qiang Sun ${ }^{18}$, Pengfei Ge ${ }^{19}$, Xiaolan Ren ${ }^{19}$, Caixia Dong ${ }^{19}$, Hui Zhang ${ }^{20}$, Enke $\mathrm{Mao}^{20}$, Xiaoping Wang ${ }^{20}$, Tao Wang ${ }^{20}$, Xi Zhang ${ }^{20}$, Ding Zhang Zhou ${ }^{21}$, Gang Zhou $^{21}$, Shixian Feng ${ }^{21}$, Ling Chang ${ }^{21}$, Lei Fan ${ }^{21}$, Yulian Gao22, Tianyou $\mathrm{He}^{22}$, Huarong Sun ${ }^{22}$, Pan $\mathrm{He}^{22}$, Chen $\mathrm{Hu}^{22}$, Xukui Zhang ${ }^{22}$, Huifang $\mathrm{Wu}^{22}$, Min $\mathrm{Yu}^{23}$, Ruying Hu${ }^{23}$, Hao Wang ${ }^{23}$, Weiwei Gong ${ }^{23}$, Meng Wang ${ }^{23}$, Kaixu Xie ${ }^{24}$, Lingli Chen ${ }^{24}$, Dongxia Pan ${ }^{24}$, Qijun Gü24, Yuelong Huang ${ }^{25}$, Biyun Chen ${ }^{25}$, Li Yin ${ }^{25}$, Huilin Liu ${ }^{25}$, Zhongxi Fu ${ }^{25}$, Qiaohua Xu² ${ }^{25}$ Xin Xu², Hao Zhang ${ }^{26}$, Huajun Long ${ }^{26}$, Libo Zhang ${ }^{26}$

BioBank Japan Project Consortium41

Akiko Nagai,

Department of Public Policy, Institute of Medical Science, The University of Tokyo, Tokyo, Japan.

\section{Kaori Muto,}

Department of Public Policy, Institute of Medical Science, The University of Tokyo, Tokyo, Japan.

\section{Koichi Matsuda,}

Laboratory of Genome Technology, Human Genome Center, Institute of Medical Science, The University of Tokyo, Tokyo, Japan. Laboratory of Clinical Genome Sequencing, Graduate School of Frontier Sciences, The University of Tokyo, Tokyo, Japan.

\section{Makoto Hirata,}

Laboratory of Genome Technology, Human Genome Center, Institute of Medical Science, The University of Tokyo, Tokyo, Japan.

\section{Takayuki Morisaki,}

Division of Molecular Pathology, Institute of Medical Science, The University of Tokyo, Tokyo, Japan.

\section{Yasushi Yamashita,}

The BioBank Japan, Tokyo, Japan.

\section{Yoichiro Kamatani,}

Laboratory of Complex Trait Genomics, Graduate School of Frontier Sciences, The University of Tokyo, Tokyo, Japan. Laboratory for Statistical and Translational Genetics, RIKEN Center for Integrative Medical Sciences, Yokohama, Japan.

\section{Yoko Kambara,}

Department of Public Policy, Institute of Medical Science, The University of Tokyo, Tokyo, Japan. 
Yoshinori Murakami,

Division of Molecular Pathology, Institute of Medical Science, The University of

Tokyo, Tokyo, Japan.

Akihide Masumoto,

Aso lizuka Hospital, Fukuoka, Japan.

Satoshi Nagayama,

Department of Gastroenterology, Cancer Institute Hospital, Japanese Foundation for Cancer Research, Tokyo, Japan.

Yoshio Miki,

Department of Genetic Diagnosis, The Cancer Institute, Japanese Foundation for Cancer Research, Tokyo, Japan.

\section{Kozo Yoshimori,}

Fukujuji Hospital, Tokyo, Japan.

Tomoaki Fujioka,

Department of Urology, Iwate Medical University School of Medicine, Iwate, Japan.

\section{Ryo Takata,}

Department of Urology, Iwate Medical University School of Medicine, Iwate, Japan.

Ken Yamaji,

Department of Internal Medicine and Rheumatology, Juntendo University Graduate School of Medicine, Tokyo, Japan.

\section{Kazuhisa Takahashi,}

Department of Respiratory Medicine, Juntendo University Graduate School of Medicine, Tokyo, Japan.

\section{Satoshi Asai,}

Division of Pharmacology, Department of Biomedical Science, Nihon University School of Medicine, Tokyo, Japan. Division of Genomic Epidemiology and Clinical Trials, Clinical Ttrials Research Center, Nihon University. School of Medicine, Tokyo, Japan.

\section{Yasuo Takahashi,}

Division of Genomic Epidemiology and Clinical Trials, Clinical Ttrials Research Center, Nihon University School of Medicine, Tokyo, Japan.

\section{Shiro Minami,}

Department of Bioregulation, Nippon Medical School, Tokyo, Japan.

\section{Hiroki Yamaguchi,}

Department of Hematology, Nippon Medical School, Tokyo, Japan.

Yukihiro Koretsune,

National Hospital Organization Osaka National Hospital, Osaka, Japan.

\section{Yasuko Nishizawa,}


Osaka International Cancer Institute (formerly, Osaka Medical Cancer Center for Cancer \& Cardiovascular Diseases), Osaka, Japan

Ken Kodama,

Osaka International Cancer Institute (formerly, Osaka Medical Cancer Center for Cancer \& Cardiovascular Diseases), Osaka, Japan

\section{Hiromu Kutsumi,}

Center for Clinical Research and Advanced Medicine, Shiga University of Medical Science, Shiga, Japan.

Takao Suzuki,

Tokushukai Group, Tokyo, Japan.

Nobuaki Sinozaki,

Tokushukai Group, Tokyo, Japan.

Shigeo Murayama,

Department of Neurology and Neuropathology (the Brain Bank for Aging Research), Tokyo Metropolitan Geriatric Hospital and Institute of Gerontology, Tokyo, Japan.

Yoichi Furukawa,

Division of Clinical Genome Research, Institute of Medical Science, The University of Tokyo, Tokyo, Japan.

\section{Yuji Yamanashi}

Division of Genetics, The Institute of Medical Science, The University of Tokyo, Minato-ku, Tokyo, Japan.

\section{The ICAN Study Group41}

Hubert Desal, Romain Bourcier, Richard Redon, Gervaise Loirand, Jean-Jacques Schott, Stéphanie Chatel, Emmanuelle Bourcereau, Christian Dina, Floriane Simonet, Eric Charpentier, Estelle Baron, Stéphanie Bonnaud, Benjamin Daumas-Duport, Bertrand Isidor, Jérôme Connault, Pierre Lebranchu, Thierry Le Tourneau University Hospital of Nantes, Nantes, France

Chrisanthi Papagiannaki,

University Hospital of Rouen, Rouen, France

Michel Piotin, Hocine Redjem, Mikael Mazighi, Jean Philippe Desilles - Fondation Rotschild Hospital, Paris, France

Olivier Naggara,

Saint Anne Hospital, Paris, France

Denis Trystram, Saint Anne Hospital, Paris, France

Myriam Edjlali-Goujon, 
Saint Anne Hospital, Paris, France

Grégoire Boulouis,

Saint Anne Hospital, Paris, France

Christine Rodriguez,

Saint Anne Hospital, Paris, France

Waghi Ben Hassen,

Saint Anne Hospital, Paris, France

Suzanna Saleme,

University Hospital of Limoges, Limoges, France

Charbel Mounayer,

University Hospital of Limoges, Limoges, France

Aymeric Rouchaud,

University Hospital of Limoges, Limoges, France

Olivier Levrier,

Clairval Hospital, Marseille, France

Pierre Aguettaz,

Clairval Hospital, Marseille, France

Xavier Combaz,

Clairval Hospital, Marseille, France

Anne Pasco,

University Hospital of Angers, Angers, France

Vincent l'Allinec,

University Hospital of Angers, Angers, France

Marc Bintner,

University Hospital of La Réunion, Saint-Denis de La Réunion, France

Marc Molho,

University Hospital of La Réunion, Saint-Denis de La Réunion, France

Gauthier Pascale,

University Hospital of La Réunion, Saint-Denis de La Réunion, France

Cyril Chivot,

University Hospital of Amiens, Amiens, France

Vincent Costalat,

University Hospital of Montpellier, Montpellier, France

Cyril Darganzil,

University Hospital of Montpellier, Montpellier, France

Alain Bonafé,

University Hospital of Montpellier, Montpellier, France 
Anne Christine Januel,

University Hospital of Toulouse, Toulouse, France

Caterina Michelozzi,

University Hospital of Toulouse, Toulouse, France

Christophe Cognard,

University Hospital of Toulouse, Toulouse, France

Fabrice Bonneville,

University Hospital of Toulouse, Toulouse, France

Philippe Tall,

University Hospital of Toulouse, Toulouse, France

Jean Darcourt,

University Hospital of Toulouse, Toulouse, France

Alessandra Biondi,

University Hospital of Besançon, Besançon, France

Cristina losif,

University Hospital of Besançon, Besançon, France

Jean Christophe Ferre,

University Hospital of Rennes, Rennes, France

Jean Yves Gauvrit,

University Hospital of Rennes, Rennes, France

François Eugene,

University Hospital of Rennes, Rennes, France

Hélène Raoult,

University Hospital of Rennes, Rennes, France

Jean Christophe Gentric,

University Hospital of Brest, Brest, France

Julien Ognard,

University Hospital of Brest, Brest, France

René Anxionnat,

University Hospital of Nancy, Nancy, France

Benjamin Gory,

University Hospital of Nancy, Nancy, France

Serge Bracard,

University Hospital of Nancy, Nancy, France

Anne Laure Derelle,

University Hospital of Nancy, Nancy, France

Romain Tonnelet, 
University Hospital of Nancy, Nancy, France

Laurent Spelle,

Le Kremlin Bicêtre Hospital, Paris, France

Léon Ikka,

Le Kremlin Bicêtre Hospital, Paris, France

Augustin Ozanne,

Le Kremlin Bicêtre Hospital, Paris, France

Sophie Gallas,

Le Kremlin Bicêtre Hospital, Paris, France

Jildaz Caroff,

Le Kremlin Bicêtre Hospital, Paris, France

Nidal Ben Achour,

Le Kremlin Bicêtre Hospital, Paris, France

Jacques Moret,

Le Kremlin Bicêtre Hospital, Paris, France

Emmanuel Chabert,

University Hospital of Clermont-Ferrand, Clermont-Ferrand, France

Jérôme Berge,

University Hospital of Brdeaux, Bordeaux, France

Gaultier Marnat,

University Hospital of Brdeaux, Bordeaux, France

Xavier Barreau,

University Hospital of Brdeaux, Bordeaux, France

Florent Gariel,

University Hospital of Brdeaux, Bordeaux, France

Frédéric Clarencon,

La Pitié Salpétrière Hospital, Paris, France

Eimad Shotar,

La Pitié Salpétrière Hospital, Paris, France

Mohammed Aggour,

University Hospital of Saint Etienne, Saint Etienne, France

Frédéric Ricolfi,

University Hospital of Dijon, Dijon, France

Adrien Chavent,

University Hospital of Dijon, Dijon, France

Pierre Thouant,

University Hospital of Dijon, Dijon, France 
Pablo Lebidinsky,

Colmar Hospital, Colmar, France

Brivael Lemogne,

Colmar Hospital, Colmar, France

Denis Herbreteau,

University Hospital of Tours, Tours, France

Richard Bibi,

University Hospital of Tours, Tours, France

Kevin Janot,

University Hospital of Tours, Tours, France

Laurent Pierot,

University Hospital of Reims, Reims, France

Sébastien Soize,

University Hospital of Reims, Reims, France

Marc Antoine Labeyrie,

Emmanuel Houdart - Lariboisière Hospital, Paris, France

Christophe Vandendries,

Emmanuel Houdart - Lariboisière Hospital, Paris, France

Appoline Kazemi,

University Hospital of Lille, Lille, France

Xavier Leclerc,

University Hospital of Lille, Lille, France

Jean Pierre Pruvo,

University Hospital of Lille, Lille, France

Nicolas Bricout,

University Hospital of Lille, Lille, France

Stéphane Velasco,

University Hospital of Poitiers, Poitiers, France

Samy Boucebci

University Hospital of Poitiers, Poitiers, France

\section{CADISP Group41}

Robin Lemmens MD, PhD,

Department of Neurology, Leuven University Hospital, and Vesalius Research Center, VIB, Leuven, Belgium

Massimo Pandolfo MD PhD, Department of Neurology, Erasme Hospital, Free University of Brussels and Laboratory of Experimental Neurology, ULB, Brussels, Belgium 
Marie Bodenant MD,

Department of Neurology, Lille University Hospital - EA1046, France

Fabien Louillet MD,

Department of Neurology, Sainte- Anne University Hospital, Paris, France

Jean-Louis Mas MD PhD,

Department of Neurology, Sainte- Anne University Hospital, Paris, France

Sandrine Deltour MD,

Department of Neurology, Pitié-Salpêtrière University Hospital, Paris, France

Sara Leder MD,

Department of Neurology, Pitié-Salpêtrière University Hospital, Paris, France

Anne Léger MD,

Department of Neurology, Pitié-Salpêtrière University Hospital, Paris, France

Sandrine Canaple MD,

Department of Neurology, Amiens University Hospital, France

Olivier Godefroy MD PhD,

Department of Neurology, Amiens University Hospital, France

Maurice Giroud MD PhD,

Department of Neurology, Dijon University Hospital, France

Agnès Jacquin MD,

Department of Neurology, Dijon University Hospital, France

Thierry Moulin MD PhD,

Department of Neurology, Besançon University Hospital, France

Fabrice Vuillier MD,

Department of Neurology, Besançon University Hospital, France

Christophe Tzourio MD PhD,

Michael Dos Santos MD,

Department of Neurology, Klinikum Ludwigshafen, Germany

Rainer Malik MD PhD,

Department of Neurology, University Hospital of Munich, Germany

Ingrid Hausser $\mathrm{PhD}$,

Department of Dermatology, Heidelberg University Hospital, Germany

Constanze Thomas-Feles MD,

Department of Rehabilitation Schmieder-Klinik, Heidelberg, Germany

Ralf Weber MD,

Department of Rehabilitation Schmieder-Klinik, Heidelberg, Germany

Caspar Grond-Ginsbach PhD,

Department of Neurology, Heidelberg University Hospital, Germany

Nat Genet. Author manuscript; available in PMC 2021 January 04. 
Werner Hacke MD PhD,

Department of Neurology, Heidelberg University Hospital, Germany

Alessia Giossi MD,

Department of Clinical and Experimental Sciences, Neurology Clinic, Brescia University Hospital, Italy

Irene Volonghi MD,

Department of Clinical and Experimental Sciences, Neurology Clinic, Brescia University Hospital, Italy

Paolo Costa MD,

Department of Clinical and Experimental Sciences, Neurology Clinic, Brescia University Hospital, Italy

Elisabetta del Zotto MD PhD,

Department of Clinical and Experimental Sciences, Neurology Clinic, Brescia University Hospital, Italy

Andrea Morotti MD,

Department of Clinical and Experimental Sciences, Neurology Clinic, Brescia University Hospital, Italy

Loris Poli MD,

Department of Clinical and Experimental Sciences, Neurology Clinic, Brescia University Hospital, Italy

Maria Lorenza Muiesan MD,

Department of Clinical and Experimental Sciences, Clinica Medica, Brescia University Hospital, Italy

Massimo Salvetti MD,

Department of Clinical and Experimental Sciences, Clinica Medica, Brescia University Hospital, Italy

Enrico Agabiti Rosei MD,

Department of Clinical and Experimental Sciences, Clinica Medica, Brescia University Hospital, Italy

Silvia Lanfranconi MD, Department of Neurology, IRCCS Foundation Ca'Granda Hospital Policlinic Hospital, University of Milan, Italy

Pierluigi Baron MD PhD, Department of Neurology, IRCCS Foundation Ca'Granda Hospital Policlinic Hospital, University of Milan, Italy

Carlo Ferrarese MD PhD, University of Milano Bicocca, San Gerardo Hospital, Monza, Italy

Emanuela Susani MD, University of Milano Bicocca, San Gerardo Hospital, Monza, Italy 
Giacomo Giacalone MD,

Milan Scientific Institute San Raffaele University Hospital, Italy

Stefano Paolucci MD PhD,

Department of Rehabilitation, Santa Lucia Hospital, Rome, Italy

Raffaele Palmirotta MD,

Department of Laboratory Medicine \& Advanced Biotechnologies, IRCCS San

Raffaele Pisana, Rome, Italy

Fiorella Guadagni MD,

Department of Laboratory Medicine \& Advanced Biotechnologies, IRCCS San

Raffaele Pisana, Rome, Italy

Maurizio Paciaroni MD PhD,

Stroke Unit and Division of Cardiovascular Medicine, University of Perugia Santa

Maria della Misericordia Hospital, Sant'Andrea delle Fratte, Perugia, Italy

Elena Ballabio MD,

Department of Cerebrovascular Diseases, and Emilio Ciusani, PhD, Laboratory of

Clinical Investigation, Fondazione IRCCS Istituto Neurologico Carlo Besta, Milano, Italy

Eugenio A. Parati MD,

Department of Cerebrovascular Diseases, and Emilio Ciusani, PhD, Laboratory of Clinical Investigation, Fondazione IRCCS Istituto Neurologico Carlo Besta, Milano, Italy

Felix Fluri MD,

Department of Neurology, Basel University Hospital, Switzerland

Florian Hatz MD,

Department of Neurology, Basel University Hospital, Switzerland

Dominique Gisler MD,

Department of Neurology, Basel University Hospital, Switzerland

Margareth Amort,

Department of Neurology, Basel University Hospital, Switzerland

Steve Bevan PhD,

Stroke and Dementia Research Centre, St George's University of London, UK

Tom James BSc,

Stroke and Dementia Research Centre, St George's University of London, UK

Sandra Olsson PhD,

Institute of Neuroscience and Physiology, the Sahlgrenska Academy at University of Gothenburg, Gothenburg, Sweden

Lukas Holmegaard MD,

Institute of Neuroscience and Physiology, the Sahlgrenska Academy at University of Gothenburg, Gothenburg, Sweden 
Ayse Altintas MD PhD,

Department of Neurology, University Hospital of Istanbul, Turkey

Juan José Martin MD,

Department of Neurology, University Hospital Sanatorio Allende, Cordoba,

Argentina

Steven Kittner MD MPH,

Maryland Stroke Center, Department of Neurology, University of Maryland School of Medicine, Baltimore, Maryland, USA

Braxton Mitchell PhD,

Maryland Stroke Center, Department of Neurology, University of Maryland School of Medicine, Baltimore, Maryland, USA

Colin Stine PhD,

Maryland Stroke Center, Department of Neurology, University of Maryland School of Medicine, Baltimore, Maryland, USA

Jeff O'Connell PhD,

Maryland Stroke Center, Department of Neurology, University of Maryland School of Medicine, Baltimore, Maryland, USA

Nicole Dueker PhD,

Maryland Stroke Center, Department of Neurology, University of Maryland School of Medicine, Baltimore, Maryland, USA

Peter J. Koudstaal MD PhD,

Department of Neurology, Erasmus MC University Medical Center, Rotterdam, the Netherlands

Lonneke M.L. de Lau MD PhD,

Department of Neurology, Erasmus MC University Medical Center, Rotterdam, the Netherlands

Albert Hofman MD PhD,

Department of Epidemiology Erasmus MC Rotterdam

Benjamin F Verhaaren MD MSc,

Department of Epidemiology Erasmus MC Rotterdam

Ralf Weber MD,

Department of Internal Medicine, Erasmus MC

Andre G Uitterlinden PhD,

Department of Internal Medicine, Erasmus MC

Joan Montaner MD PhD,

Laboratorio Neurovascular, Institut de Recerca, Hospital Vall d'Hebron, Barcelona, Spain

Maite Mendioroz MD PhD, 
Laboratorio Neurovascular, Institut de Recerca, Hospital Vall d'Hebron, Barcelona, Spain

Sunaina Yadav MSc, Imperial College Cerebrovascular Research Unit (ICCRU), Imperial College London, UK

Muhammad Saleem Khan BSc,

Imperial College Cerebrovascular Research Unit (ICCRU), Imperial College London, UK

Michael Wilder MD,

University of Utah, USA

Ewoud van Dijk MD PhD,

Imperial College Cerebrovascular Research Unit (ICCRU), Imperial College London, UK

Noortje Maaijwee MD, Imperial College Cerebrovascular Research Unit (ICCRU), Imperial College London, UK

Loes Rutten-Jacobs MSc,

Donders Institute for Brain, Cognition and Behaviour, Centre for Neuroscience, Department of Neurology, Radboud University Nijmegen Medical Centre, Nijmegen, The Netherlands

Jamie Kramer BA, Departments of Neurology and Public Health Sciences University of Virginia, Charlottesville, Virginia, USA

Shaneela Malik MD, Henry Ford Hospital, Detroit, Michigan, USA

Ralf Weber MD, Henry Ford Hospital, Detroit, Michigan, USA

Thomas G Brott MD, Department of Neurology, Mayo Clinic, Jacksonville, Florida, USA

Robert D Brown Jr MD, Department of Neurology, Mayo Clinic, Rochester, Minnesota, USA

Andrew Singleton $\mathrm{PhD}$, Molecular Genetics Section, Laboratory of Neurogenetics, National Institute on Aging, NIH

John Hardy PhD, Department of Molecular Neuroscience Institute of Neurology, University College London, London, United Kingdom

Stephen S Rich PhD, 
Department of Public Health Sciences and the Center for Public Health Genomics, University of Virginia, Charlottesville, Virginia, USA

Christian Tanislav MD PhD,

Department of Neurology, University Hospital of Giessen, Germany

Jan Jungehülsing

Charité Universitätsmedizin Berlin, Department of Neurology, Berlin, Germany.

Genetics and Observational Subarachnoid Haemorrhage (GOSH) Study

investigators41

David Werring Prof,

UCL Queen Square Institute of Neurology, London, UK

Henry Houlden Prof,

UCL Queen Square Institute of Neurology, London, UK

Varinder Alg Mr,

UCL Queen Square Institute of Neurology, London, UK

Isabel Hostettler Dr,

UCL Queen Square Institute of Neurology, London, UK

Stephen Bonner Dr,

The James Cook University Hospital, Middlesbrough, UK

Daniel Walsh Mr,

King's College Hospital NHS Foundation Trust, London, UK

Diederik Bulters Mr,

University Hospital Southampton NHS Foundation Trust, Southampton UK

Neil Kitchen Mr,

The National Hospital of Neurology and Neurosurgery, London, UK

Martin Brown Prof,

UCL Queen Square Institute of Neurology, London, UK

Joan Grieve Ms,

The National Hospital of Neurology and Neurosurgery, London, UK

Joan Grieve Ms,

The National Hospital of Neurology and Neurosurgery, London, UK

Gareth Roberts Mr,

Royal Preston Hospital, UK

Timothy Jones Mr,

St George's Hospital, London, UK

Giles Critchley Mr,

Hurstwood Park Neurological Centre, UK 
Pankaj Sharma Prof,

Imperial Healthcare, Charing Cross Hospital, London, UK

Richard Nelson Mr,

Frenchay Hospital, Bristol, UK

Peter Whitfield Prof,

Derriford Hospital, Plymouth, UK

Stuart Ross Mr,

Leeds General Infirmary, UK

Hiren Patel Mr,

Salford Royal Hospital, UK

Paul Eldridge Mr,

The Walton Centre, Liverpool, UK

Kari Saastamoinen Dr,

The Royal London Hospital, London, UK

Umang Patel Mr,

Sheffield Teaching Hospitals NHS Trust, UK

Enas Lawrance Dr,

Mayday Hospital, Croydon, UK

Subha Vandabona Dr,

Mount Gould Hospital, Plymouth, UK

David Mendelow Professor,

Newcastle University Hopsitals NHS Trust

Rachel Teal Ms,

Oxford Radcliffe Infirmary, UK

Orlando Warner Dr,

Oxford Radcliffe Infirmary, UK

Peter Kirkpatrick Professor

Addenbrooke's Hospital, Cambridge, UK

International Stroke Genetics Consortium (ISGC)41

Sudha Seshadri,

Department of Neurology,Boston University School of Medicine, Framingham Heart

Study, Boston, MA, USA

Laura Kilarski,

University of Cologne,Germany

Hyacinth I Hyacinth,

Aflac Cancer and Blood Disorder Center, Department of Pediatrics, Emory

University School of Medicine, Atlanta, GA, USA 
Jamary Oliveira,

Filho Stroke and Cardiomyopathy Clinics,Federal University of Bahia, Brazilian

National Research Committee (CNPq), Instituto Nacional de Ciencia e

Tecnologiaem Doencas Tropicais (INCT-DT),Salvador,Brazil

Sandro Marini,

Center for Human Genetic Research,Harvard Medical School,Boston, MA,USA

Sunaina Yadav, Imperial College Cerebrovascular Research Unit, Department of

Clinical Neuroscience,Imperial College London,London,UK

Paul Nyquist,

Medicine,Baltimore, MD,USA Department of Anesthesiology and Critical Care

Medicine,Johns Hopkins

Cathryn Lewis,

Department of Medical and Molecular Genetics, King's College London, SGDP

Centre, Institute of Psychiatry, Psychology \& Neuroscience, King's College

London,London,UK Bjorn Hansen, Department of Clinical Sciences Lund,

Neurology,Lund University,Department of Neurology and Rehabilitation Medicine,

Skåne University Hospital,Lund,Sweden

Bo Norrving,

Sahlgrenska Academy at University of Gothenburg, Lund University,Department of Neurology and Rehabilitation Medicine,Skåne University Hospital,Lund,Sweden

Gustav Smith,

Department of Clinical Sciences Lund, Cardiology,Lund University, The Section for Heart Failure and Valvular Disease, VO Heart and Lung medicine,Skane University Hospital,Lund,Sweden

Jonathan Rosand,

Department of Neurology,Massachusetts General Hospital,Center for Human

Genetic Research,Massachusetts General Hospital (MGH),Boston, MA,USA

Alessandro Biffi,

Division of Behavioral Neurology, Department of Neurology,Massachusetts General Hospital,Center for Human Genetic Research,Massachusetts General Hospital (MGH),Boston, MA,USA

Christina Kourkoulis, Department of Neurology,Massachusetts General Hospital,Center for Human Genetic Research,Massachusetts General Hospital (MGH),Boston, MA,USA

Chris Anderson,

Center for Genomic Medicine,Massachusetts General Hospital (MGH),J. Philip

Kistler Stroke Research Center, Department of Neurology, Massachusetts General Hospital (MGH),Boston, MA,USA

Anne-Katrin Giese,

Department of Neurology,Massachusetts General Hospital, Harvard Medical School, Department of Neurology,J. Philip Kistler Stroke Research Center, 
MGH,Boston, MA,USA Ralph Sacco,Department of Neurology,Miller School of Medicine, University of Miami,Miami, FL,USA

Pankaj Sharma, Institute of Cardiovascular Research, Royal Holloway University of London,London,UK

Oh Young Bang, Department of Neurology,Samsung Medical Center, Sungkyunkwan University School of Medicine, Seoul, Korea

Jong-Won Chung, Department of Neurology,Samsung Medical Center, Sungkyunkwan University School of Medicine, Seoul, USA

Gyeong-Moon Kim, Department of Neurology,Samsung Medical Center, Sungkyunkwan University School of Medicine, Seoul, Korea

Qishuai Zhuang, Department of Pharmacology,School of Life Science and Biopharmaceutical Sciences, Shenyang Pharmaceutical University, Shenyang,China

Wayne Sheu,

Department of Internal Medicine,Taichung Veterans General Hospital, Taichung,Taiwan Steven Lubitz,Department of Cardiology,Massachusetts General Hospital, Harvard Medical School,Boston, MA,USA

David Werring,

Stroke Research Centre, Department of Brain Repair and Rehabilitation,University College London Institute of Neurology,London,UK

John Hardy, Reta Lila Weston Institute, UCL Institute of Neurology,University College London,London,UK

June Smalley, Reta Lila Weston Institute, UCL Institute of Neurology,University College London,London,UK

Romain Bourcier, Department of Neuroradiology,University Hospital of Nantes,Nantes,France Joanna Howson, MRC/BHF Cardiovascular Epidemiology Unit, Department of Public Health and Primary Care,University of Cambridge,Cambridge,UK

Alessandra Granata,

Department of Clinical Neurosciences, University of Cambridge,Cambridge,UK Anna Drazyk,Department of Clinical Neurosciences, University of Cambridge,Cambridge,UK 
Hugh Markus,

Stroke Research Group, Division of Clinical Neurosciences, University of Cambridge, Cambridge,UK

Joanna Wardlaw,

Neuroimaging Sciences, University of Edinburgh, Centre for Clinical Brain

Sciences,University of Edinburgh, Edinburgh,UK

Braxton Mitchell,

Department of Medicine,University of Maryland School of Medicine, Geriatrics

Research and Education Clinical Center,Baltimore, Veterans Administration Medical

Center,Baltimore, MD,USA

John Cole,

Department of Neurology,University of Maryland School of Medicine and Baltimore, VAMC,Baltimore, MD,USA

Anbupalam Thalamuthu,

Centre for Healthy Brain Ageing, Psychiatry,University of New South Wales

(UNSW),Sydney,Australia

Jemma Hopewell,

Clinical Trial Service Unit and Epidemiological Studies Unit, Nuffield Department of

Population Health,University of Oxford,Oxford,UK

Robin Walters,

Clinical Trial Service Unit and Epidemiological Studies Unit, Nuffield Department of Population Health,University of Oxford,Oxford,UK

lain Turnbull,

Clinical Trial Service Unit and Epidemiological Studies Unit, Nuffield Department of Population Health,University of Oxford,Oxford,UK

Bradford Worrall,

Departments of Neurology and Public Health Sciences,University of Virginia School of Medicine,Charlottesville, VA,USA

Josh Bis,

Cardiovascular Health Research Unit, Department of Medicine,University of

Washington, Seattle, WA,USA

David Tirschwell,

Comprehensive Stroke Care at the UW Medicine Stroke Center at Harborview Medical Center,University of Washington, Seattle, WA,USA

Alex Reiner,

Department of Epidemiology,University of Washington, Fred Hutchinson Cancer

Research Center, University of Washington, Seattle, WA,USA

Raj Dhar, 
Department of Neurology,Washington University School of Medicine,St. Louis, MO,USA Laura Heitsch,Department of Neurology, Radiology, and Biomedical Engineering,Washington University School of Medicine,St. Louis, MO,USA

Jin-Moo Lee,

Department of Neurology, Radiology, and Biomedical Engineering, Washington University School of Medicine,St. Louis, MO,USA

Janne Mortenson, Department of Neurology,Aarhus University Hospital,Aarhus, Denmark

Sylvia Wassertheil-Smoller, Department of Epidemiology and Population Health,Albert Einstein College of Medicine,New York, NY,USA

Kameshwar Prasad,

Department of Neurology and Stroke Unit;and Director of Clinical Epidemiology,,All India Institute of Medical Sciences (AIIMS),New Delhi,India

Mark Fisher, Department of Neurology,University of Massachusetts Medical School, Worchester, MA,USA

Christopher Traenka,

Neurology Clinic,Basel University Hospital,Basel,Switzerland

Xingwu Wang,

Beijing Hypertension League Institute,Beijing,China

Yongjun Wang,

Center of Stroke,Beijing Insitute for Brain Disorders,Department of Neurology,

Beijing Tiantan Hospital, Capital Medical University,Beijing,China

Francois Rouanet,

Department of Neurology,Bordeaux University Hospital,Bordeaux,France

Igor Sibon,

Department of Neurology,Bordeaux University Hospital,Bordeaux,France

Chloé Sarnowski,

Department of Biostatistics,Boston University,Boston,USA

Pauline Maillard,

Department of Neurology,University of California at Davis,Sacramento, CA,USA

Aleksandra Pikula,Department of Neurology,University of Toronto,Toronto,USA

Hugo Javier Aparicio,

Department of Neurology,Boston University School of Medicine,Boston,USA Philip

Wolf,Boston University School of Medicine,Boston,USA

Josee Dupuis,

Boston University School of Public Health,Boston,USA

Qiong Yang, 
Boston University School of Public Health,Boston,USA

Gustavo Luvizutto,

Rehabilitation department,Botucatu Medical School,Botucatu School of

Medicine,University Estadual Paulista Júlio de Mesquita Filho, District of Rubião

Junior,Botucatu,Brazil

Daniel Chasman,

Division of Preventive Medicine,Brigham and Women's Hospital,Harvard Medical

School,Boston, MA,USA

Kathryn Rexrode,

Department of Medicine,Brigham and Women's Hospital,Harvard Medical

School,Boston,USA

Andrea Harriot,

Department of Neurology,Brigham and Women's Hospital, Massachusetts General Hospital,Boston, MA,USA

Chia-Ling Phuah,

Center for Human Genetic Research, Massachusetts General Hospital

(MGH),Division of Neurocritical Care and Emergency Neurology, Department of

Neurology,Boston, MA,USA

Gustavo Santo,

Stroke Unit,Centro Hospitalar e Universitário de Coimbra, CHUC,Coimbra,Portugal

Ales Tomek,Department of Neurology, 2nd Faculty of Medicine,Charles University in

Prague and Motol University Hospital,Prague,Czech Republic

Jen Gerard,

Department of Cardiology,Children's Hospital of Wisconsin,Milwaukee,USA Cara

Carty,Children's Research Institute,Children's National Medical Center,Center for

Translational Science,George Washington University, Washington,USA

Guiyou Liu,

Chinese Academy of Sciences (CAS),Beijing, China

Sanjith Aaron,

Department of Neurological Sciences,Christian Medical College,Vellore,India

Christhunesa S. Christudass,

Department of Neurological Sciences,Christian Medical College,Vellore,India

BSB Salomi,

Department of Neurological Sciences,Christian Medical College,Vellore,India

Dharambir Sanghera,

Department of Pediatrics, College of Medicine, University of Oklahoma Health

Sciences Center,Department of Pharmaceutical Sciences, College of Pharmacy, University of Oklahoma Health Sciences Center,Oklahoma City,USA

Amelia Boehme, 
Department of Neurology,Columbia University,New York,USA

Mitchell Elkind,

Neurological Institute of New York,Columbia University College,New York,USA

Gudmar Thorleifsson,deCODE genetics/AMGEN inc,Reykjavik,Iceland

Solveig Gretarsdottir,

deCODE genetics/AMGEN inc, Reykjavik,Iceland

Leslie Lange,

University of Colorado Denver Anschutz Medical Campus,Denver, CO,USA

Natalia Rost,

Department of Neurology, Massachusetts General Hospital, Harvard Medical

School, Boston, MA, USA,Department of Neurology, J. Philip Kistler Stroke

Research Center, MGH,Boston, MA,USA

Michael James,

Translational Acute Brain Injury Research Center,Duke University,Durham,USA

Jill Stewart,

Department of Neurology,Duke University School of Medicine,Durham,USA

Larry Goldstein,

Department of Medicine, Duke Stroke Center,Duke Univesrity School of

Medicine,Durham, NC, USA

Salina Waddy,

Cardiovascular and Stroke Genetics Working Group,Emory

University,Atlanta,USA Carla Ibrahim-Verbaas,Department of Neurology,Erasmus

MC, Rotterdam,Netherlands

Dina Vojinovic,

Department of Epidemiology,Erasmus MC, University Medical Center

Rotterdam,Clinic for Neurology and Psychiatry for Children and Youth,University of

Belgrade, Faculty of Medicine,Rotterdam,Netherlands

Arfan Ikram,

Department of Epidemiology,Erasmus University Medical

Center,Rotterdam,Netherlands Hieab Adams,Department of Epidemiology,Erasmus

University Medical Center,Rotterdam,Netherlands Fadi Charchar,L.E.W.

Carty Cardiovascular Genomics laboratory,Federation University

Australia,Ballarat,Australia

Vincent Thijs,

Stroke Division,Florey Institute of Neuroscience and Mental Health,Department of Neurology,Austin Health,Heidelberg,Australia

Eugenio Parati, Laboratory of Cellular Neurobiology,Fondazione IRCCS Istituto Neurologico C. Besta, Cerebrovascular Disease Unit,Cerebrovascular Disease Unit,Fondazione IRCCS Istituto Neurologico C Besta,Milan, Italy 
Giorgio Boncoraglio,

Department of Cerebrovascular Diseases,Fondazione IRCCS Istituto Neurologico

Carlo Besta,Milan,Italy

Charles Kooperberg,

Division of Public Health Sciences, Fred Hutchinson Cancer Research

Center,Seattle,USA

Sherrine Abboud,

Laboratory of Experimental Neurology,Free University of Brussels

(ULB),Brussels,Belgium

Ramin Zand,

Department of Neuroscience,Geisinger Health System,Danville,USA

Philippe Bijlenga,

Departement des neurosciences cliniques, Clinique de neurochirurgie,Geneva

University Hospital,Geneva,Switzerland

Magdy Selim,

Beth Israel Deaconess Medical Center,Harvard Medical School,Boston,USA

caspar Grond-Ginsbach,Department of Neurology,Heidelberg University

Hospital,Heidelberg,Germany

Olli Happola,

Department of Neurology,Helsinki University Central Hospital, Helsinki,Finland

Daniel Strbian,

Department of Neurology,Helsinki University Central Hospital, Clinical

Neurosciences, Neurology,University of Helsinki,Helsinki,Finland

Liisa Tomppo,

Department of Neurology,Helsinki University Central Hospital, Helsinki,Finland

Hanne Sallinen,Department of Neurology,Helsinki University Hospital,

Helsinki,Finland

Abhishek Pathak,

Department of Neurology,Heritage Hospital,Department of Neurology,Neuro Expert

Clinic,Varanasi,India

Dorothea Pfeiffer,

Department of Neurology,Hiedelberg University Hospital,Heidelberg,Germany Pablo

Bonardo,Department of Neurology,Hospital Británico

de Buenos Aires,

Ciudad Autónoma de Buenos Aires,Argentina

Joao Jose Freitas de Carvalho,

Hospital Geral de Fortaleza/SUS Fortaleza,Ceará,Brazil

Priscila Ribeiro,

Hospital Governador Celso Ramos,Florianópolis,Brazil 
Nuria Torres,

Stroke Pharmacogenomics and Genetics,Hospital Mútua de Terrassa, Fundació Docència i Recerca Mútua Terrassa,Terrassa,Spain

Miguel Barboza, Department of Neurology,Hospital Rafael A. Calderon Guardia,San Jose,Costa Rica Melanie Laarman,Cardiac Development and Genetics Group,Hubrecht Institute,Utrecht,Netherlands Roberta Carriero,Inflammation and immunology laboratory, Humanitas Clinical Institute,Pavia, Italy Elizabeth Holliday,Public Health Stream,Hunter Medical Research Institute,Faculty of Health and Medicine,University of Newcastle,New Lambton,Australia

Androniki Plomaritoglou, Neurology,Hygeia Hospital,Marousi,Greece

Johan Bjorkegren, Department of Genetic and Genomic Sciences, Icahn School of Medicine,New York,USA

Yu-Feng Yvonne Chan, Department of Genetic and Genomic Sciences, Icahn School of Medicine, Mount Sinai,New York,USA

Villi Gudnason, Icelandic Heart Association Research Institute,Faculty of Medicine,University of Iceland,Kopavogur, Iceland

Jordi Jimenez-Conde, Neurovascular Research Group (NEUVAS). Neurology Department., IMIM Hospital del Mar,Barcelona,Spain

Carolina Soriano, Department of Neurology, Neurovascular Research Group,IMIM-Hospital del Mar,Barcelona,Spain

Jaume Roquer, Neurology Service,IMIM-Hospital del Mar, Institut Hospital del Mar d'investigacions Mèdiques,Barcelona,Spain

Paul Bentley, Faculty of Medicine Department of Medicine, Imperial College London,London,UK Dipender Gill,Centre for Pharmacology and Therapeutics, Imperial College London, London,UK Ganesh Chauhan,Centre for Brain Research,Indian Institute of Science,Bangalore,India

Elisabeth Tournier-Lasserve, UMR-S 740,INSERM,Paris,France Sara Kaffashian,INSERM U1219 Bordeaux Population Health Research Center,Bordeaux,France Cecilia Samieri,INSERM U1219 Bordeaux Population Health Research Center,ISPED,University of Bordeaux,Bordeaux,France 
Carole Dufouil,

INSERM U1219 Bordeaux Population Health Research Center,Department of

Public Health, CHU Bordeaux,Bordeaux,France

Stephanie Debette,

INSERM U1219 Bordeaux Population Health Research Center, Bordeaux,

France,University of Bordeaux,France

Aniket Mishra,

INSERM U1219 Bordeaux Population Health Research Center, Bordeaux, France,University of Bordeaux,France

Lawrence Wee,

Data Analytics Department,Institute for Infocomm Research

(I2R),Singapore,Singapore

Saima Siddiqi,

Institute of Biomedical And Genetic Engineering,Islamabad,Pakistan

Jer-Yuarn Wu,

Institute of Biomedical Sciences, Academia Sinica, Taipei,Taiwan

Tai-Ming Ko,

Institute of Biomedical Sciences, Academia Sinica, Graduate Institute of Integrated

Medicine, China Medical University,Taipei,Taiwan

Silvia Bione,

Computational Biology Unit,Institute of Molecular Genetics-National Research

Council,Pavia,Italy

Katarina Jood,

Department of Clinical Neuroscience, Institute of Neuroscience and Physiology, Sahlgrenska Academy at University of Gothenburg, Gothenburg,Sweden

Turgut Tatlisumak, Department of Clinical Neuroscience, Institute of Neuroscience and Physiology, Sahlgrenska Academy at University of Gothenburg, Sahlgrenska University Hospital,Gothenburg,Sweden

Lukas Holmegaard,

Department of Clinical Neuroscience, Institute of Neuroscience and Physiology, Sahlgrenska Academy at University of Gothenburg, Gothenburg,Sweden

Antonio Arauz,

Stroke Clinic,Instituto Nacional de Neurologia y Neurocirugia, Manuel Velasco

Suarez,Mexico City,Mexico

Michal Korostynski,

Department of Molecular Neuropharmacology,Polish Academy of

Sciences, Warsaw,Poland

Lenore Launer, 
Laboratory of Epidemiology and Population Science, Intramural Research Program, National Institutes of Health, National Institute on Aging,Bethesda,USA

Suo Yue, Ion Torrent by Life Technologies (company),Carnegie Mellon University,San Francisco,USA

anna bersano, Cerebrovascular Unit,IRCCS Foundation C. Besta Neurological Institute,Milan,Italy Tomasz Dziedzic,Department of Neurology,Jagiellonian University,Krakow,Poland

Karol Józef Juchniewicz, Jagiellonian University,Krakow,Poland

Adamski Mateusz, Jagiellonian Centre for Experimental Therapeutics,Jagiellonian University, Krakow,Poland

Joanna Pera,

Department of Neurology,Jagiellonian University Medical College,Krakow,Poland Agnieszka Slowik,Department of Neurology,Jagiellonian University Medical College,Krakow,Poland Andrzej Urbanik, Department of Radiology,Jagiellonian University Medical College,Krakow,Poland Jerzy Gasowski,Department of Internal Medicine and Gerontology,Jagiellonian University Medical College,Krakow,Poland Rafa? Olszanecki,Faculty of Medicine,Jagiellonian University Medical College,Krakow,Poland

Marcin Wnuk, Jagiellonian University Medical College,Poland

Christopher Levi, John Hunter Hospital, Hunter Medical Research Institute and University of Newcastle,Newcastle,Australia

Aaron Gusdon, Department of Neurology,Johns Hopkins Medicine,University of Pittsburgh School of Medicine,Baltimore,USA

Konstantinos Kostulas, Department of Clinical Neuroscience, Karolinska Institute,Stockholm,Sweden Milita Crisby,Division of Clinical Geriatrics, Neurobiology Care Sciences and Society,Karolinska Institute,Huddinge,Sweden Lina Keller,Karolinska Institutet,Stockholm,Sweden Kristina Schlicht,Institut für Medizinische Informatik und Statistik,Kiel University,Kiel,Germany

Jessye Maxwell,

Social genetic and Developmental Psychiatry,King's College London,London, Christof Haffner,Institute for Stroke and Dementia Research,Klinikum der Universität München,Munich,Germany

Marco Duering, 
Institute for Stroke and Dementia Research,Klinikum der Universität München, Ludwig-Maximilians-University LMU,Munich,Germany

Jeremiasz Jagiella,

Krakow University Hospital,Krakow,Poland

Robin Lemmens,

Department of Neurosciences, Experimental Neurology,KU Leuven - University of Leuven,Department of Neurology,University Hospitals Leuven, VIB Center for Brain \& Disease Research, Leuven,Belgium

Jun Hata,

Department of Epidemiology and Public Health,Kyushu University, Graduate School of Medical Sciences,Fukuoka,Japan

Toshiharu Ninomiya,

Department of Epidemiology and Public Health,Kyushu University, Graduate School of Medical Sciences, Fukuoka,Japan

Vinh Nguyen,

School of Psychological Science,La Trobe University,Melbourne,Australia

Bjorn Logi Thorarinsson,

Department of Neurology,Landspítali Háskólasjúkrahús, Reykjavík,Iceland Saskia

Lesnik Oberstein,Department of Clinical Genetics,Leiden University Medical

Center,Leiden,Netherlands

Tsong-Hai Lee,

Neurology department and Stroke center, Linkou Chang Gung Memorial

Hospital,Taipei,Taiwan

Alexandr Rakitko,

Lomonosov Moscow State University,Moscow,Russia

Rainer Malik,

Institute for Stroke and Dementia Research,Ludwig-Maximilians-University, Klinikum der Universität München,Munich,Germany

Martin Dichgans,

Institute for Stroke and Dementia Research,Ludwig-Maximilians-University,

Klinikum der Universität München, Munich Cluster for Systems Neurology

(SyNergy),Munich,Germany

Arne Lindgren,

Department of Clinical Sciences Lund, Neurology,Lund University,Department of Neurology and Rehabilitation Medicine,Skåne University Hospital,Lund,Sweden

Johan Wasselius,

Department of Clinical Sciences Malmö,Lund University,Vascular Center,Skåne University Hospital,Malmö,Sweden

Mattias Drake, 
Diagnostic Radiology,Lund University,Lund,Sweden Olle Melander,Department of

Clinical Sciences,Lund University,Lund,sweden

Martin Stenman,

Geriatric Medicine,Lund University,Lund,Sweden

Andreea llinca,

Neurology,Lund University,Lund,Sweden

Julie Staals,

Department of Neurology,Maastricht University Medical Center (MUMC+),

Cardiovascular Research Institute Maastricht (CARIM),Maastricht,Netherlands

Ariane Sadr-Nabavi,

Medical Genetics Research Center (MGRC),Mashhad University of Medical

Sciences (MUMS), School of Medicine,Department of Medical Genetics,Mashhad

University of Medical Sciences (MUMS), Faculty of Medicine,Mashhad,Iran

Katherine Crawford,

Center for Genomic Medicine, Massachusetts General Hospital,Boston, MA,USA

Jose Florez,Center for Human Genetic Research, MGH,Massachusetts General

Hospital, Harvard Medical School, Boston, MA,USA

Umme Lena,

Massachusetts General Hospital,South Hadley, MA,USA

Farrah Mateen,

Department of Neurology,Massachusetts General Hospital,Boston, MA,USA

Hakan Ay,

Department of Neurology,Massachusetts General Hospital,Boston, MA,USA

Ona Wu,

Department of Neurology, JPK Stroke Research Center,Massachusetts General

Hospital and Harvard Medical School, Department of Radiology, Athinoula A

Martinos Center for Biomedical Imaging, MGH,Boston, MA,USA

Markus Schirmer,

Department of Neurology, J. Philip Kistler Stroke Research Centre,Massachusetts General Hospital and Harvard Medical School,Boston,USA

Javier Romero,

Division of Neurocritical Care and Emergency Neurology, Department of

Neurology,Massachusetts General Hospital, Harvard Medical School,J. P. Kistler

Stroke Research Center,Massachusetts General Hospital, Harvard Medical

School,Boston, MA,USA

Steve Cramer,

Neurology, Anatomy \& Neurobiology, and Physical Medicine \&

Rehabilitation,University of California Irvine,Irvine,USA

Polina Golland, 
The Computer Science and Artificial Intelligence Laboratory, Massachusetts Institute of Technology,Cambridge,USA

Bertram Mueller,

Myhsok Max-Planck-Institute of Psychiatry,Munich,Germany

Robert Brown,

Department of Neurology,Mayo Clinic,Rochester, MN,USA

James Meschia,

Department of Neurology,Mayo Clinic,Jacksonville,USA

Owen A. Ross,

Department of Medical Genetics and Neuroscience,Mayo Clinic,Jacksonville,USA

Thomas Brott,Neurology,Mayo Clinic, Department of Surgery, University of Medicine and Dentistry of New Jersey,Jacksonville, FL,USA

Guillaume Pare,

Population Health Research Institute,McMaster University,Hamilton,Canada

Mike Chong,

Population Health Research Institute,McMaster University,Hamilton,Canada

Ossama yassin mansour,

Department of Neurology,Medical school of Alexandria,Alexandria

Governorate,Egypt

Bartosz Karaszewski,

Department of Adult Neurology,Medical University of Gdansk \& University Clinical

Centre in Gdansk,Gdansk,Poland

Christian Enzinger,

Department of Neurology,Medical University of Graz,Graz,Austria Helena

Schmidt,Institute of Molecular Biology and Biochemistry,Medical University of

Graz,Graz,Austria

Reinhold Schmidt,

Department of Neurology,Medical University of Graz,Graz,Austria

Stephan Seiler,

Department of Neurology,Medical University of Graz,Graz,Austria

Alexander Pichler,

Department of Neurology,Medical University of Graz,Graz,Germany

Bruce Ovbiagele,

Department of Neurology,Medical University of South Carolina,Charleston,USA

Yoshiji Yamada,

Department of Human Genomics,Mie University, Life Scinece Research

Center,Mie,Japan

Tatjana Rundek, 
Department of Neurology,Miller School of Medicine, University of Miami,Miami, FL,USA

Susan Blanton, Hussman Institute for Human Genomics,Miller School of Medicine, University of Miami,Miami, FL,USA

John $\mathrm{P}$, Hussman Institute for Human Genomics,Miller School of Medicine, University of Miami,Miami, FL,USA

Joseph Chern,

Department of Neurology, College of Medicine,National Cheng Kung

University,Tainan,Taiwan

Chris O'Donnell,

Cardiovascular Epidemiology and Human Genomics Branch,National Heart, Lung and Blood Institute, Intramural Research,Veterans Administration Healthcare,Center for Population Genomics,Boston, MA,USA

Roderick Corriveau,

National Institute of Neurological Disorders and Stroke,National Institute of Health, University of California,Bethesda, MD,USA

Pallab Bhattacharya,

National Institute of Pharmaceutical Education and Research,Ahmedabad,India Andrew Singleton,Laboratory of Neurogenetics,National Institute on Aging, Intramural Research Program,Bethesda, MD,USA

Katrina Gwinn, National Institute of Neurological Disorders and Stroke,National Institutes of Health,Bethesda, MD,USA

BHARATENDU CHANDRA, General neurology and stroke,National University Hospital,Singapore,Singapore Christopher Chen, Department of Pharmacology,National University of Singapore,Singapore,Singapore

Raj Kalaria, Institute of Neuroscience,Newcastle University,Newcastle, UK

Jim Koenig,

Division of Neuroscience, Stroke Program in the Neural Environment, NINDS,Bethesda, MA,USA

Om Prakash Singh, Department of Environmental Studies,North Eastern Hill University (NEHU),Shillong,India

Akintomi Olugbodi, 
Obafemi Awolowo University,lle-Ife,Nigeria Bishwa Sapkota,Harold Hamm Diabetes Center,Oklahoma University,Oklahoma City,USA Rebecca Jackson,Center for women's health, Ohio State University Wexner Medical Center,Colombus,USA

Eva Giralt,

Parc de Salut Mar,Barcelona,Spain

Danish Saleheen,

Department of Genetics,Perelman School of Medicine, University of Pennsylvania,Pennsylvania, PA,USA

Frank-Erik de Leeuw, Department of Neurology,Radboud University Medical

Center,Nijmegen,Netherlands

Karin Klijn,

Department of Neurology,Radboud University Medical Centre, Donders Institute for Brain, Cognition and Behaviour,Department of Neurology and Neurosurgery,University Medical Center, Brain Center Rudolf Magnus,Nijmegen,Netherlands

Jes Olesen,

Department of Clinical Medicine,Rigshospitalet - Neurocentret,Glostrup,Denmark

Yoichiro Kamatani,

Laboratory for Statistical Analysis, RIKEN Center for Integrative Medical

Sciences, Center for Genomic Medicine, Kyoto University Graduate School of

Medicine,Yokohama,Japan

Michiaki Kubo,

RIKEN Center for Integrative Medical Sciences, Yokohama,Japan Yukinori

Okada,Laboratory for Statistical Analysis,RIKEN Center for Integrative Medical

Sciences,Department of Statistical Genetics, Osaka University Graduate School of Medicine,Yokohama,Japan

David Spence,

Stroke Prevention \& Atherosclerosis Research Centre,Robarts Research,London (ON), Canada

Annie Pedersen,

Västra Vötalandsregionen,Sahlgrenska Academy at University of Gothenburg,Gothenburg,Sweden

Maja Olsson, Department of Medical and Clinical Genetics, Institute of Biomedicine,Sahlgrenska Academy at University of Gothenburg, Gothenburg,Sweden

Turgut Tatlisumak, Department of Clinical Neurosciences/Neurology,Sahlgrenska Academy at University of Gothenburg, Institute of Neuroscience and Physiology,Sahlgrenska University Hospital,Gothenburg,Sweden 
Giacomo Giacalone,

Neurology Department,San Raffaele Scientific Institute,University Vita-Salute San Raffaele,Milan,Italy

Juan José Martín,

Department of Neurology,Sanatorio Allende,Cordoba,Argentina Rodrigo

Bazan,Department of Neurology,São Paulo State University (Unesp) Botucatu

Medical School,Botucatu,Brazil

Gabriel Braga,

Hospital das Clínicas,São Paulo State University (Unesp) Botucatu Medical

School,Botucatu,Brazil

Steve Bevan,

School of Life Science, University of Lincoln,Lincoln,UK

Huichun Xu,

Medicine department,School of Medicine, University of Maryland,Baltimore,USA

Tim Assimes,

Cardiovascular Medicine,School of Medicine, University of Stanford,Stanford,USA Jamie Wright, Anderson Cancer Center, MD Anderson Clinical Cancer

Genetics,School of Medicine, University of Texas,Houston,USA

Anton Raskurazhev,

Scientific Center of Neurology,Moscow,Russia

Wei Ling Lee,

National Neuroscience Institute,Singapore General Hospital,Department of Pediatrics,National University of Singapore, Yong Loo Lin School of

Medicine,Singapore,Singapore Eng King Tan,Department of Neurology,Singapore General Hospital, National Neuroscience Institute,Neuroscience and Behavioral Disorders Program,Duke-National University of Singapore Graduate Medical School,Singapore,Singapore

Philippe Burri,

Department of Cardiology,Skane University Hospital,Malmö,Sweden

Petrea Frid,

Critical Care Neurology,Skåne University Hospital, Lund university,Malmö,Sweden Christian Opherk,Department of Neurology,SLK-Kliniken

Heilbronn $\mathrm{GmbH}$, Heilbronn,Germany Chaeyoung Lee,Department of Bioinformatics and Life Science,Soongsil University,Seoul,Korea David Tregouet,Team Genomics and Pathophysiology of Cardiovascular Diseases,Sorbonne Universités, UPMC Univ Paris 06, INSERM, UMR_S 1166,ICAN Institute for Cardiometabolism and Nutrition,Paris, France

Zhen Deng, 
Department of Neurology,Southern Medical University, FIMMU,Guangzhou,China Hung Yi Chiou,School of Public Health, College of Public Health and Nutrition,Taipei Medical University, Taipei,Taiwan

Mahdi Habibi-koolaee, Department of Health Information Management,Tehran University of Medical Sciences (TUMS), Tehr?n,Iran

Murali Vijayan,

Garrison Institute on Aging,Texas Tech University Health Sciences

Center,Lubbock,USA

Thomas Leung,

Division of Neurology, Department of Medicine and Therapeutics, The Chinese University of Hong Kong,Hong-Kong,Hong-Kong

Lawrence Wong,

Division of Neurology, Department of Medicine and Therapeutics, The Chinese University of Hong Kong,Hong-Kong,Hong-Kong

Vincent Mok,

Department of Medicine and Therapeutics, The Chinese University of Hong Kong,Hong- Kong,Hong-Kong

Richard Choy, Department of Obstetrics and Gynecology, The Chinese University of Hong Kong,Hong-Kong,Hong-Kong

Christina Jern,

Department of Pathology and Genetics, The Sahlgrenska Academy at University of Gothenburg, Institute of Biomedicine,Gothenburg,Sweden

Elena Lebedeva,

Department of Neurology and Neurosurgery, The Urals State Medical

University,International Headache Center 'Europe-Asia', Yekaterinburg,Russia

Martin Farrall,

Department of Cardiovascular Medicine, The Wellcome Trust Centre for Human Genetics,Oxford,UK

Xu Jiayuan,

Department of radiology,Tianjin Medical University (TIJMU), Tianjin,China

Keat Wei Loo,

Tunku Abdul Rahman University, UTAR,Kampar Perak,Malaysia

Isabel Hostettler, Stroke Research Center,UCL Institue of Neurology,London,UK

Gabriel Rinkel, Department of Neurology and Neurosurgery,UMC Utrecht, Brain Center Rudolf Magnus, 
Utrecht,Netherlands

Anderson Goncalves,

Universidade da Região de Joinville,Joinville,Brazil

Paulo Franca,

Department of Medicine,Universidade da Região de Joinville -

Univille,Joinville,Brazil Norberto Cabral, Department of Medicine,Universidade da

Região de Joinville (Univille), Joinville,Brazil

Iscia Cendes,

Molecular Genetics Laboratory,Universidade Estadual de Campinas, Faculdade de Ciências Médicas,Campinas,Brazil

Caty Carrera,

Neurovascular Research Laboratory, Vall d'Hebron Institut of Research, Neurology and Medicine Departments,Universitat Autònoma de Barcelona, Vall d'Hebrón Hospital,Stroke Pharmacogenomics and Genetics,Fundacio Docència i Recerca Mutua Terrassa,Spain

Israel Fernandez-Cadenas, Neurovascular Research Laboratory, Vall d'Hebron Institut of Research, Neurology and Medicine Departments,Universitat Autònoma de Barcelona, Vall d'Hebrón Hospital,Stroke Pharmacogenomics and Genetics,Fundacio Docència i Recerca Mutua Terrassa,Barcelona,Spain

Joan Montaner, Neurovascular Research Laboratory,Universitat Autònoma de Barcelona, Vall d'Hebron Institute of Research,Neurology Department,Hospital Vall d'Hebron,Barcelona,Spain

Helen Kim, Institute for Human Genetics, The Center for Cerebrovascular Research (CCR),Université de Californie,San Francisco,USA

Arndt Rolfs,

Albrecht Kossel Institute,University Clinic of Rostock,Rostock,Germany

Mayowa Owolabi, Department of Neurology,University College Hospital,Ibadan,Nigeria Reecha Sofat,Metabolism \& Experi Therapeutics, Div of Medicine, Faculty of Medical Sciences,University College London,London,UK

Mark Bakker, University Medical Center Utrecht,Utrecht,Netherlands Dominique PV de Kleijn,Laboratory of Experimental Cardiology,University Medical Center Utrecht,ICIN-Netherlands Heart Institute,Utrecht,Netherlands

Ynte Ruigrok, 
Department of Neurology and Neurosurgery,University Medical Center Utrecht, Brain Center Rudolf Magnus, The Dutch Parelsnoer Institute-Cerebrovascular accident (CVA) Study Group,Utrecht,Netherlands

Allard Hauer,

Department of Neurology and Neurosurgery,University Medical Center Utrecht, Brain Center Rudolf Magnus,Utrecht,Netherlands

Sara L. Pulit,

Department of Neurology,University Medical Center Utrecht, Brain Center

Rudolf Magnus,

Utrecht,Netherlands

Ale Algra,

Department of Neurology and Neurosurgery,University Medical Center Utrecht, Brain Center Rudolf Magnus, Julius Center for Health Sciences and Primary

Care,University Medical Center Utrecht,Utrecht,Netherlands

Sander W. van der Laan,

Laboratory of Experimental Cardiology,University Medical Center Utrecht, Division

of Heart and Lungs, Utrecht,Netherlands

Mary Macleod,

Dept of Medicine \& Therapeutics,University of Aberdeen, King's

College,Aberdeen,UK

George Howard,

School of Public Health,University of Alabama,Birmingham, AL,USA

Hemant Tiwari,

Department of Biostatistics,University of Alabama,Birmingham, AL,USA

Ryan Irvin,

Department of Epidemiology,University of Alabama at Birmingham,Birmingham, AL,USA

Karen C. Albright,

Neurology,University Of Alabama Hospital, Spectrum Health Butterworth

Campus,Birmingham, AL,USA

Rodney Perry,

Department of Epidemiology,University of Alabama School of Medicine,Birmingham, AL,USA

Chelsea Kidwell, Department of Neurology,University of Arizona,Stroke Center at Georgetown,University of Georgetown,Tucson, AZ,USA

Aleksandra Pavlovic, Neurology Clinic, Clinical Center of Serbia,University of Belgrade, Faculty of Medicine,Belgrade, Serbia 
Christophe Tzourio,

University of Bordeaux,INSERM U1219,Bordeaux,France

Murali Sargurupremraj,

INSERM U1219 Bordeaux Population Health Research Center, University of

Bordeaux,Bordeaux,France

Sabrina Schilling,

University of Bordeaux,Bordeaux, Alessandro Padovani,Department of Clinical and Experimental Sciences, University of Brescia,Brescia,Italy

Alessandro Pezzini,

Department of Clinical and Experimental Sciences,University of Brescia, Neurology

Clinic,Brescia,Italy

Foad Abd-Allah,

Department of Neurology,University of Cairo,Cairo,Egypt

Charles DeCarli,

Alzheimer's Disease Center,University of Californa Davis,Sacramento, CA, USA

Yuqi Zhao,Department of Integrative Biology and Physiology,University of California, Los Angeles, CA,USA

David Liebeskind,

Department of Neurology,University of California, Los Angeles, CA,USA

Matthew Traylor,

Stroke Research Group, Division of Clinical Neurosciences, University of

Cambridge,Cambridge,UK

Rhea Tan,

Department of Clinical Neurosciences,University of Cambridge,Cambridge,England

John Danesh,

MRC/BHF Cardiovascular Epidemiology Unit, Department of Public Health and

Primary Care,, University of Cambridge, The National Institute for Health Research

Blood and Transplant Research Unit in Donor Health and Genomics, Wellcome

Trust Sanger Institute,Cambridge,UK

Susanna C. Larsson,

Department of Clinical Neurosciences, Neurology Unit,University of

Cambridge,Cambridge,UK

Loes Rutten,

Jacobs,Stroke Research Group,University of Cambridge, Division of Clinical

Neurosciences,DZNE,Cambdrige,UK

Amanda Donatti,

School of Medical Sciences, FCM,University of Campinas, UNICAMP,São

Paulo,Brazil

Wagner Avelar, 
Department of Neurology,University of Campinas, UNICAMP,São Paulo,Brazil Joseph Broderick, Gardner Neuroscience Institute, Comprehensive Stroke Center,University of Cincinnati,Cincinnati, OH,USA

Daniel Woo, University of Cincinnati College of Medicine,Cincinnati, $\mathrm{OH}, \mathrm{USA}$

Brett Kissela, Department of Neurology and Rehabilitation Medicine,University of Cincinnati Gardner Neuroscience Institute, Comprehensive Stroke Center,Cincinnati, $\mathrm{OH}, \mathrm{USA}$ Laura Garcia Ibenez, Institute for Cancer Genetics,University of Columbia,New York,USA

Rustam Salman,

Centre for Clinical Brain Sciences, University of Edinburgh, Edinburgh,UK

Cathie Sudlow,

Centre for Medical Informatics, Usher Institute, University of Edinburgh,

Edinburgh,UK

Kristiina Rannikmäe,

Centre for Medical Informatics, Usher Institute,University of

Edinburgh,Edinburgh,UK

Caitrin Wheeler McDonough,

College of Pharmacy,University of Florida,Gainesville, FL,USA

Scott Silliman,

Department of Neurology,University of Florida College of Medicine,Jacksonville, FL,USA

Oyunbileg Magvanjav, Department of Pharmacotherapy and Translational Research and Center for Pharmacogenomics, University of Florida, College of Pharmacy,Gainesville, FL,Japan USA

Tom van Agtmael, Insittute of Cardiovascular and Medical Sciences,University of Glasgow,Glasgow,UK Matthew Walters, School of Medicine, Dentistry and Nursing,University of Glasgow,Glasgow,UK Martin Söderholm,Bioinformatics Core Facility,University of Gothenburg,Gothenburg,Sweden

Erik Lorentzen, Bioinformatics Core Facility,University of Gothenburg,Gothenburg,Sweden

Sandra Olsson, University of Gothenburg,Gothenburg,Sweden

Tara Stanne, 
University of Gothenburg,Gothenburg,Sweden

Martina Olsson,

University of Gothenburg,Gothenburg,Sweden

Kazuma Nakagawa,

University of Hawaii, John A. Burns School of Medicine,Department of

Neurology,The Queen's Medical Center,Honolulu,USA

Rufus Akinyemi,

Institute for Advanced Medical Research and Training,University of Ibadan, College

of Medicine, Ibadan,Nigeria

loana Cotlatciuc,

Institute of Cardiovascular Research Royal Holloway,University of London,

London,UK

Jeff O'Connell,

Medicine department,University of Maryland,Animal Genetics Improvement

Laboratory,United States Department of Agriculture,Baltimore, MD,USA

Mary Sparks,

Department of Neurology,University of Maryland,Baltimore, MD,USA

John Sorkin,

Department of Neural and Pain Sciences, University of Maryland,Baltimore, MD,USA

Patrick McArdle,Division of Endocrinology, Diabetes and Nutrition,University of

Maryland School of Medicine,Baltimore, MD,USA

Tushar Dave,

Bioinformatics, University of Maryland School of Medicine,Baltimore, MD,USA

Colin Stine,

Epidemiology \& Public Health,University of Maryland School of Medicine,Baltimore, MD,USA

Steven Kittner,

Department of Neurology,University of Maryland School of Medicine and Baltimore, VAMC, MD, USA,Baltimore, MD,USA

Jill Naylor,

University of Melbourne,Melbourne,Australia

Devin Brown,

Department of Neurovascular,University of Michigan Medical School,Ann Arbor,USA Mario Di Napoli,Department of Neurology,University of Naples,Naples,Italy

Rose Du,

Comprehensive Cancer Center,University of New Mexico, Albuquerque, NM,USA

Tobias B. Kulik,

Neurology,University of New Mexico,Albuquerque, NM, USA

John Attia, 
School of Medicine and Public Health,University of Newcastle,New Lambton,Australia Shahbaz Zamani,University of Newcastle,Newcastle,Australia James E Faber,

Cell Biology and Physiology,University of North Carolina,Chapel Hill,USA

Peter Rothwell, Nuffield Department of Clinical Neurosciences,University of Oxford,Oxford,UK

Iona Millwood, Nuttfield Department of Population Health,University of Oxford,Oxford,UK

Elsa Valdés Márquez, CTSU-Clinical Trial Service Unit and Epidemiological Studies Unit,University of Oxford,Oxford,UK

Michelangelo Mancuso, Department of Neurology,University of Pisa,Pisa, Italy

Doralina Brum Souza, Department of Neurology,University of Sao Paulo,Ribeirao Preto,Brazil

Ranil de Silva,

Genetic, Diagnostic and Research Laboratory, Department of Anatomy,University of Sri Jayewardenepura, Faculty of Medical Sciences,Nugegoda,Sri Lanka

Riina Vibo,

Department of Neurology and Neurosurgery,University of Tartu,Tartu,Estonia Janika Korv, Department of Neurology and Neurosurgery,University of Tartu,Tartu,Estonia Jane Maguire, Faculty of Health,University of Technology Sydney,Ultimo,Australia

Myriam Fornage,

Brown Foundation Institute of Molecular Medicine // Human Genetics Center,University of Texas Health Science Center at Houston,Houston, TX,USA

Kachikwu Illoh, University of Texas Medical School,Houston, TX,USA

Dianna Milewicz, Division of Medical Genetics,University of TexasHealth Science Center, McGovern Medical School,Department of Internal Medicine,University of TexasHealth Science Center, McGovern Medical School,Houston, TX,USA

Jennifer Majersik, Neurology,University of Utah,Salt Lake City, UT,USA

Adam DeHavenon, Department of Neurology,University of Utah,Salt Lake City, UT,USA

Yashar Kalani, 
Neurobiology and Anatomy, University of Utah School of Medicine,Salt Lake City, UT,USA

Matthew Alexander,

Radiology and Imaging Sciences, University of Utah School of

Medicine,Neurosurgery, University of Utah School of Medicine,Salt Lake City, UT,USA

Mary Cushman,

Department of Hematology and oncology,University of Vermont, Medical

Center,Colchester,USA

Michele Sale,

Center for Public Health Genomics,University of Virginia,Charlottesville,

VA,USA Andrew Southerland, Departments of Neurology and Public Health

Sciences,University of Virginia,Charlottesville, VA,USA

Debra Owens,

Newborn Intensive Care Units,University of Virginia Children's

Hospital,Charlottesville, VA,USA

Keith Keene,

Center for Public Health Genomics,University of Virginia School of

Medicine,Charlottesville, VA,USA

Stephe Rich,

Center for Public Health Genomics, University of Virginia School of

Medicine,Charlottesville, VA,USA

Bruce Psaty,

Cardiovascular Health Research Unit, Department of Medicine,University of

Washington,Department of Epidemiology,University of Washington,Seattle, WA,USA

Will Longstreth,

Department of Epidemiology,University of Washington,Department of

Neurology,University of Washington,Seattle,USA

Masharip Atadzhanov,

Department of Neurology,University Teaching Hospital,Lusaka,Zambia

Stacey Quintero Wolfe,

Wake Forest Baptist Health,Winston-Salem, NC, USA

Carl Langefeld,

Center for Public Health Genomics and Department of Biostatistical Sciences, Wake

Forest School of Medicine,Winston-Salem, NC,USA

Cheryl Bushnell,

Department of Neurology,Wake Forest University Medical Center,Winston-Salem, NC,USA

Carlos Cruchaga, 
Department of Psychiatry, The Hope Center Program on Protein Aggregation and Neurodegeneration (HPAN),Washington University School of Medicine,Department of Developmental Biology, Washington University School of Medicine,St. Louis, MO,USA

Jan Konrad,

Department of Psychiatry, The Hope Center Program on Protein Aggregation and Neurodegeneration (HPAN), Washington University, School of Medicine,St. Louis, MO,USA

Junfeng Liu,

West China Medical School, Sichuan University,Sichuan,China

Kevin Sheth,

Department of Neurology, Neuro Intensive Care \& Neurological Emergencies, Yale School of Medicine,Nw Haven, CT,USA

Guido Falcone,

Department of Neurology, Yale University School of Medicine, Program in Medical and Population Genetics, The Broad Institute of Harvard and MIT,New Haven, CT, USA

Kathleen Donahue J

Philip Kistler Stroke Research Center,Massachusetts General Hospital,USA

Gregory T. Jones ${ }^{42}$, Matthew J. Bown ${ }^{43,44}$, Nerissa U. Ko ${ }^{45}$, Helen $\mathrm{Kim}^{46,47,48}$, Jonathan R.I. Coleman ${ }^{49,50}$, Gerome Breen49,50, Jonathan G. Zaroff $^{51}$, Catharina J.M. Klijn ${ }^{52}$, Rainer Malik ${ }^{53}$, Martin Dichgans ${ }^{53,54,55}$, Muralidharan Sargurupremraj ${ }^{56,57}$, Turgut Tatlisumak ${ }^{58}$, Philippe Amouyel ${ }^{59}$, Stéphanie Debette ${ }^{56,57}$, Gabriel J.E. Rinkel ${ }^{1}$, Bradford B. Worrall ${ }^{60}$, Joanna Pera $^{61}$, Agnieszka Slowik ${ }^{61}$, Emilia I. Gaál-Paavola ${ }^{62,63}$, Mika Niemelä62, Juha E. Jääskeläinen 64,65, Mikael von Und Zu Fraunberg64,65, Antti Lindgren64,65, Joseph P. Broderick $^{66}$, David J. Werring ${ }^{6}$, Daniel Woo ${ }^{66}$, Richard Redon ${ }^{4}$, Philippe Bijlenga ${ }^{3}$, Yoichiro Kamatani ${ }^{13}$, Jan H. Veldink ${ }^{1, \dagger}$, Ynte M. Ruigrok ${ }^{1,{ }^{*}, \dagger}$

Amy E Martinsen ${ }^{1}$, Anne Hege Aamodt ${ }^{2}$, Anne Heidi Skogholt ${ }^{3,4}$, Else Charlotte Sandset $^{5,6}$, Espen S Kristoffersen ${ }^{7,8}$, Hanne Ellekjaer ${ }^{9,10}$, Ingrid Heuch $^{8}$, Jonas Bille Nielsen ${ }^{3,11,12}$, Knut Hagen ${ }^{13,14}$, Lars Fritsche ${ }^{15}$, Laurent F. Thomas ${ }^{3,4}$, Linda Pedersen ${ }^{8}$, Maiken E Gabrielsen ${ }^{3}$, Maria Dehli Vigeland,

Oddgeir Holmen ${ }^{16}$, Wei Zhou

Junshi Chen ${ }^{1}$, Zhengming Chen (PI) ${ }^{2,3}$, Robert Clarke ${ }^{2}$, Rory Collins ${ }^{2}$, Yu Guo ${ }^{4}$, Liming Li (PI) ${ }^{5}$, Depei Liu ${ }^{4}$, Jun Lv ${ }^{5}$, Richard Peto ${ }^{2}$, Robin Walters ${ }^{2,3}$, Daniel Avery ${ }^{2}$, Ruth Boxall ${ }^{2,3}$, Derrick Bennett ${ }^{2}$, Yumei Chang ${ }^{2}$, Yiping Chen ${ }^{2,3}$, Zhengming Chen $^{2,3}$, Robert Clarke ${ }^{2}$, Huaidong Du, ${ }^{2,3}$, Wei Gan², Simon Gilbert ${ }^{2}$, Alex Hacker ${ }^{2}$, Michael Hill2,3, Michael Holmes ${ }^{2,3}$, Andri lona ${ }^{2}$, Christiana Kartsonaki ${ }^{2,3}$, Rene Kerosi $^{2}$, Ling Kong ${ }^{2}$, Garry Lancaster ${ }^{2}$, Sarah Lewington ${ }^{2,3}$, Kuang Lin ${ }^{2}$, John 
McDonnell ${ }^{2}$, Iona Millwood ${ }^{2,3}$, Qunhua $\mathrm{Nie}^{2}$, Paul Ryder², Sam Sansome ${ }^{2}$, Dan Schmidt-Valle ${ }^{2}$, Paul Sherliker ${ }^{2}$, Rajani Sohoni ${ }^{2}$, Becky Stevens ${ }^{2}$, lain Turnbull², Robin Walters ${ }^{2,3}$, Lin Wang ${ }^{2}$, Neil Wright ${ }^{2}$, Ling Yang ${ }^{2,3}$, Xiaoming Yang ${ }^{2}$, Pang $\mathrm{Yao}^{2}$, Zheng Bian ${ }^{4}$, Yu Guo ${ }^{4}$, Xiao Han ${ }^{4}$, Can Hou${ }^{4}$, Jun Lv ${ }^{5}$, Pei Pei ${ }^{4}$, Chao $\mathrm{Liu}^{4}$, Canqing $\mathrm{Yu}^{4}$, Zengchang Pang ${ }^{6}$, Ruqin $\mathrm{Gao}^{6}$, Shanpeng $\mathrm{Li}^{6}$, Shaojie Wang ${ }^{6}$, Yongmei Liu', Ranran Du', Liang Cheng ${ }^{6}$, Xiaocao Tian ${ }^{6}$, Hua Zhang ${ }^{6}$, Yaoming Zhai $^{6}$, Feng Ning ${ }^{6}$, Xiaohui Sun ${ }^{6}$, Feifei Li ${ }^{6}$, Silu Lv ${ }^{7}$, Junzheng Wang ${ }^{7}$, Wei Hou ${ }^{7}$, Mingyuan Zou ${ }^{8}$, Shichun Yan ${ }^{8}$, Xue Zhou ${ }^{8}$, Bo Yu ${ }^{9}$, Yanjie Li ${ }^{9}$, Qinai Xu ${ }^{9}$, Quan Kang $^{9}$, Ziyan Guo ${ }^{9}$, Dan Wang ${ }^{10}$, Ximin $\mathrm{Hu}^{10}$, Jinyan Chen ${ }^{10}$, Yan $\mathrm{Fu}^{10}$, Xiaohuan Wang $^{10}$, Min Weng ${ }^{11}$, Zhendong Guo ${ }^{11}$, Shukuan Wu${ }^{11}$, Yilei Li ${ }^{11}$, Huimei Li ${ }^{11}$, Ming Wu ${ }^{11}$, Yonglin Zhou ${ }^{12}$, Jinyi Zhou ${ }^{12}$, Ran Tao ${ }^{12}$, Jie Yang ${ }^{12}$, Jian Su ${ }^{12}$, Fang $\mathrm{liu}^{13}$, Jun Zhang ${ }^{13}$, Yihe $\mathrm{Hu}^{13}$, Yan Lu ${ }^{13}$, Liangcai Ma ${ }^{13}$, Aiyu Tang ${ }^{13}$, Yujie Hua ${ }^{13}$, Jianrong Jin ${ }^{14}$, Jingchao Liu ${ }^{14}$, Zhenzhu Tang ${ }^{15}$, Naying Chen ${ }^{15}$, Ying Huang ${ }^{15}$, Mingqiang Li ${ }^{16}$, Jinhuai Meng ${ }^{16}$, Rong Pan ${ }^{16}$, Qilian Jiang ${ }^{16}$, Jian Lan ${ }^{16}$, Yun Liu' ${ }^{16}$, Liuping Wei ${ }^{16}$, Liyuan Zhou ${ }^{16}$, Ningyu Chen ${ }^{16}$, Ping Wang ${ }^{16}$, Fanwen Meng ${ }^{16}$, Yulu Qin Sisi Wang ${ }^{16}$, Xianping Wu ${ }^{17}$, Ningmei Zhang ${ }^{17}$, Xiaofang Chen ${ }^{17}$, Weiwei Zhou $^{17}$, Guojin Luo ${ }^{18}$, Jianguo Li ${ }^{18}$, Xiaofang Chen ${ }^{18}$, Xunfu Zhong ${ }^{18}$, Jiaqiu Liu ${ }^{18}$, Qiang Sun ${ }^{18}$, Pengfei $\mathrm{Ge}^{19}$, Xiaolan Ren ${ }^{19}$, Caixia Dong ${ }^{19}$, Hui Zhang ${ }^{20}$, Enke $\mathrm{Mao}^{20}$, Xiaoping Wang ${ }^{20}$, Tao Wang ${ }^{20}$, Xi Zhang ${ }^{20}$, Ding Zhang Zhou ${ }^{21}$, Gang Zhou $^{21}$, Shixian Feng ${ }^{21}$, Ling Chang ${ }^{21}$, Lei Fan ${ }^{21}$, Yulian Gao ${ }^{22}$, Tianyou $\mathrm{He}^{22}$, Huarong Sun ${ }^{22}$, Pan $\mathrm{He}^{22}$, Chen $\mathrm{Hu}^{22}$, Xukui Zhang ${ }^{22}$, Huifang $\mathrm{Wu}^{22}$, Min $\mathrm{Yu}^{23}$, Ruying $\mathrm{Hu}^{23}$, Hao Wang ${ }^{23}$, Weiwei Gong ${ }^{23}$, Meng Wang ${ }^{23}$, Kaixu Xie ${ }^{24}$, Lingli Chen ${ }^{24}$, Dongxia Pan ${ }^{24}$, Qijun Gu${ }^{24}$, Yuelong Huang ${ }^{25}$, Biyun Chen ${ }^{25}$, Li Yin ${ }^{25}$, Huilin Liu ${ }^{25}$, Zhongxi Fu25, Qiaohua Xu²5, Xin Xu²6, Hao Zhang ${ }^{26}$, Huajun Long ${ }^{26}$, Libo Zhang 26

\section{Akiko Nagai,}

Department of Public Policy, Institute of Medical Science, The University of Tokyo, Tokyo, Japan.

\section{Kaori Muto,}

Department of Public Policy, Institute of Medical Science, The University of Tokyo, Tokyo, Japan.

\section{Koichi Matsuda,}

Laboratory of Genome Technology, Human Genome Center, Institute of Medical Science, The University of Tokyo, Tokyo, Japan. Laboratory of Clinical Genome Sequencing, Graduate School of Frontier Sciences, The University of Tokyo, Tokyo, Japan.

\section{Makoto Hirata,}

Laboratory of Genome Technology, Human Genome Center, Institute of Medical Science, The University of Tokyo, Tokyo, Japan.

\section{Takayuki Morisaki,}

Division of Molecular Pathology, Institute of Medical Science, The University of Tokyo, Tokyo, Japan.

\section{Yasushi Yamashita,}


The BioBank Japan, Tokyo, Japan.

Yoichiro Kamatani,

Laboratory of Complex Trait Genomics, Graduate School of Frontier Sciences, The University of Tokyo, Tokyo, Japan. Laboratory for Statistical and Translational Genetics, RIKEN Center for Integrative Medical Sciences, Yokohama, Japan.

Yoko Kambara,

Department of Public Policy, Institute of Medical Science, The University of Tokyo, Tokyo, Japan.

\section{Yoshinori Murakami,}

Division of Molecular Pathology, Institute of Medical Science, The University of Tokyo, Tokyo, Japan.

Akihide Masumoto,

Aso lizuka Hospital, Fukuoka, Japan.

Satoshi Nagayama,

Department of Gastroenterology, Cancer Institute Hospital, Japanese Foundation for Cancer Research, Tokyo, Japan.

Yoshio Miki,

Department of Genetic Diagnosis, The Cancer Institute, Japanese Foundation for Cancer Research, Tokyo, Japan.

Kozo Yoshimori,

Fukujuji Hospital, Tokyo, Japan.

Tomoaki Fujioka,

Department of Urology, Iwate Medical University School of Medicine, Iwate, Japan.

Ryo Takata,

Department of Urology, Iwate Medical University School of Medicine, Iwate, Japan.

Ken Yamaji,

Department of Internal Medicine and Rheumatology, Juntendo University Graduate School of Medicine, Tokyo, Japan.

Kazuhisa Takahashi,

Department of Respiratory Medicine, Juntendo University Graduate School of Medicine, Tokyo, Japan.

Satoshi Asai,

Division of Pharmacology, Department of Biomedical Science, Nihon University School of Medicine, Tokyo, Japan. Division of Genomic Epidemiology and Clinical Trials, Clinical Ttrials Research Center, Nihon University. School of Medicine, Tokyo, Japan.

Yasuo Takahashi,

Division of Genomic Epidemiology and Clinical Trials, Clinical Ttrials Research Center, Nihon University School of Medicine, Tokyo, Japan. 


\section{Shiro Minami,}

Department of Bioregulation, Nippon Medical School, Tokyo, Japan.

Hiroki Yamaguchi,

Department of Hematology, Nippon Medical School, Tokyo, Japan.

Yukihiro Koretsune,

National Hospital Organization Osaka National Hospital, Osaka, Japan.

Yasuko Nishizawa,

Osaka International Cancer Institute (formerly, Osaka Medical Cancer Center for Cancer \& Cardiovascular Diseases), Osaka, Japan

Ken Kodama,

Osaka International Cancer Institute (formerly, Osaka Medical Cancer Center for Cancer \& Cardiovascular Diseases), Osaka, Japan

Hiromu Kutsumi,

Center for Clinical Research and Advanced Medicine, Shiga University of Medical Science, Shiga, Japan.

Takao Suzuki,

Tokushukai Group, Tokyo, Japan.

Nobuaki Sinozaki,

Tokushukai Group, Tokyo, Japan.

Shigeo Murayama,

Department of Neurology and Neuropathology (the Brain Bank for Aging Research), Tokyo Metropolitan Geriatric Hospital and Institute of Gerontology, Tokyo, Japan.

Yoichi Furukawa,

Division of Clinical Genome Research, Institute of Medical Science, The University of Tokyo, Tokyo, Japan.

\section{Yuji Yamanashi}

Division of Genetics, The Institute of Medical Science, The University of Tokyo, Minato-ku, Tokyo, Japan.

Hubert Desal, Romain Bourcier, Richard Redon, Gervaise Loirand, Jean-Jacques Schott, Stéphanie Chatel, Emmanuelle Bourcereau, Christian Dina, Floriane Simonet, Eric Charpentier, Estelle Baron, Stéphanie Bonnaud, Benjamin Daumas-Duport, Bertrand Isidor, Jérôme Connault, Pierre Lebranchu, Thierry Le Tourneau University Hospital of Nantes, Nantes, France

Chrisanthi Papagiannaki,

University Hospital of Rouen, Rouen, France

Michel Piotin, Hocine Redjem, Mikael Mazighi, Jean Philippe Desilles - Fondation Rotschild Hospital, Paris, France 
Olivier Naggara,

Saint Anne Hospital, Paris, France

Denis Trystram,

Saint Anne Hospital, Paris, France

Myriam Edjlali-Goujon,

Saint Anne Hospital, Paris, France

Grégoire Boulouis,

Saint Anne Hospital, Paris, France

Christine Rodriguez,

Saint Anne Hospital, Paris, France

Waghi Ben Hassen,

Saint Anne Hospital, Paris, France

Suzanna Saleme,

University Hospital of Limoges, Limoges, France

Charbel Mounayer,

University Hospital of Limoges, Limoges, France

Aymeric Rouchaud,

University Hospital of Limoges, Limoges, France

Olivier Levrier,

Clairval Hospital, Marseille, France

Pierre Aguettaz,

Clairval Hospital, Marseille, France

Xavier Combaz,

Clairval Hospital, Marseille, France

Anne Pasco,

University Hospital of Angers, Angers, France

Vincent l'Allinec,

University Hospital of Angers, Angers, France

Marc Bintner,

University Hospital of La Réunion, Saint-Denis de La Réunion, France

Marc Molho,

University Hospital of La Réunion, Saint-Denis de La Réunion, France

Gauthier Pascale,

University Hospital of La Réunion, Saint-Denis de La Réunion, France

Cyril Chivot,

University Hospital of Amiens, Amiens, France

Vincent Costalat, 
University Hospital of Montpellier, Montpellier, France

Cyril Darganzil,

University Hospital of Montpellier, Montpellier, France

Alain Bonafé,

University Hospital of Montpellier, Montpellier, France

Anne Christine Januel,

University Hospital of Toulouse, Toulouse, France

Caterina Michelozzi,

University Hospital of Toulouse, Toulouse, France

Christophe Cognard,

University Hospital of Toulouse, Toulouse, France

Fabrice Bonneville,

University Hospital of Toulouse, Toulouse, France

Philippe Tall,

University Hospital of Toulouse, Toulouse, France

Jean Darcourt,

University Hospital of Toulouse, Toulouse, France

Alessandra Biondi,

University Hospital of Besançon, Besançon, France

Cristina losif,

University Hospital of Besançon, Besançon, France

Jean Christophe Ferre,

University Hospital of Rennes, Rennes, France

Jean Yves Gauvrit,

University Hospital of Rennes, Rennes, France

François Eugene,

University Hospital of Rennes, Rennes, France

Hélène Raoult,

University Hospital of Rennes, Rennes, France

Jean Christophe Gentric,

University Hospital of Brest, Brest, France

Julien Ognard,

University Hospital of Brest, Brest, France

René Anxionnat,

University Hospital of Nancy, Nancy, France

Benjamin Gory,

University Hospital of Nancy, Nancy, France 
Serge Bracard,

University Hospital of Nancy, Nancy, France

Anne Laure Derelle,

University Hospital of Nancy, Nancy, France

Romain Tonnelet,

University Hospital of Nancy, Nancy, France

Laurent Spelle,

Le Kremlin Bicêtre Hospital, Paris, France

Léon Ikka,

Le Kremlin Bicêtre Hospital, Paris, France

Augustin Ozanne,

Le Kremlin Bicêtre Hospital, Paris, France

Sophie Gallas,

Le Kremlin Bicêtre Hospital, Paris, France

Jildaz Caroff,

Le Kremlin Bicêtre Hospital, Paris, France

Nidal Ben Achour,

Le Kremlin Bicêtre Hospital, Paris, France

Jacques Moret,

Le Kremlin Bicêtre Hospital, Paris, France

Emmanuel Chabert,

University Hospital of Clermont-Ferrand, Clermont-Ferrand, France

Jérôme Berge,

University Hospital of Brdeaux, Bordeaux, France

Gaultier Marnat,

University Hospital of Brdeaux, Bordeaux, France

Xavier Barreau,

University Hospital of Brdeaux, Bordeaux, France

Florent Gariel,

University Hospital of Brdeaux, Bordeaux, France

Frédéric Clarencon,

La Pitié Salpétrière Hospital, Paris, France

Eimad Shotar,

La Pitié Salpétrière Hospital, Paris, France

Mohammed Aggour,

University Hospital of Saint Etienne, Saint Etienne, France

Frédéric Ricolfi, 
University Hospital of Dijon, Dijon, France

Adrien Chavent,

University Hospital of Dijon, Dijon, France

Pierre Thouant,

University Hospital of Dijon, Dijon, France

Pablo Lebidinsky,

Colmar Hospital, Colmar, France

Brivael Lemogne,

Colmar Hospital, Colmar, France

Denis Herbreteau,

University Hospital of Tours, Tours, France

Richard Bibi,

University Hospital of Tours, Tours, France

Kevin Janot,

University Hospital of Tours, Tours, France

Laurent Pierot,

University Hospital of Reims, Reims, France

Sébastien Soize,

University Hospital of Reims, Reims, France

Marc Antoine Labeyrie,

Emmanuel Houdart - Lariboisière Hospital, Paris, France

Christophe Vandendries,

Emmanuel Houdart - Lariboisière Hospital, Paris, France

Appoline Kazemi,

University Hospital of Lille, Lille, France

Xavier Leclerc,

University Hospital of Lille, Lille, France

Jean Pierre Pruvo,

University Hospital of Lille, Lille, France

Nicolas Bricout,

University Hospital of Lille, Lille, France

Stéphane Velasco,

University Hospital of Poitiers, Poitiers, France

Samy Boucebci

University Hospital of Poitiers, Poitiers, France

Robin Lemmens MD, PhD, 
Department of Neurology, Leuven University Hospital, and Vesalius Research Center, VIB, Leuven, Belgium

Massimo Pandolfo MD PhD,

Department of Neurology, Erasme Hospital, Free University of Brussels and Laboratory of Experimental Neurology, ULB, Brussels, Belgium

Marie Bodenant MD,

Department of Neurology, Lille University Hospital - EA1046, France

Fabien Louillet MD,

Department of Neurology, Sainte- Anne University Hospital, Paris, France

Jean-Louis Mas MD PhD,

Department of Neurology, Sainte- Anne University Hospital, Paris, France

Sandrine Deltour MD,

Department of Neurology, Pitié-Salpêtrière University Hospital, Paris, France

Sara Leder MD,

Department of Neurology, Pitié-Salpêtrière University Hospital, Paris, France

Anne Léger MD,

Department of Neurology, Pitié-Salpêtrière University Hospital, Paris, France

Sandrine Canaple MD,

Department of Neurology, Amiens University Hospital, France

Olivier Godefroy MD PhD,

Department of Neurology, Amiens University Hospital, France

Maurice Giroud MD PhD,

Department of Neurology, Dijon University Hospital, France

Agnès Jacquin MD,

Department of Neurology, Dijon University Hospital, France

Thierry Moulin MD PhD,

Department of Neurology, Besançon University Hospital, France

Fabrice Vuillier MD,

Department of Neurology, Besançon University Hospital, France

Christophe Tzourio MD PhD,

Michael Dos Santos MD,

Department of Neurology, Klinikum Ludwigshafen, Germany

Rainer Malik MD PhD,

Department of Neurology, University Hospital of Munich, Germany

Ingrid Hausser PhD,

Department of Dermatology, Heidelberg University Hospital, Germany

Constanze Thomas-Feles MD,

Department of Rehabilitation Schmieder-Klinik, Heidelberg, Germany

Nat Genet. Author manuscript; available in PMC 2021 January 04. 
Ralf Weber MD,

Department of Rehabilitation Schmieder-Klinik, Heidelberg, Germany

Caspar Grond-Ginsbach PhD,

Department of Neurology, Heidelberg University Hospital, Germany

Werner Hacke MD PhD,

Department of Neurology, Heidelberg University Hospital, Germany

Alessia Giossi MD,

Department of Clinical and Experimental Sciences, Neurology Clinic, Brescia

University Hospital, Italy

Irene Volonghi MD,

Department of Clinical and Experimental Sciences, Neurology Clinic, Brescia

University Hospital, Italy

Paolo Costa MD,

Department of Clinical and Experimental Sciences, Neurology Clinic, Brescia

University Hospital, Italy

Elisabetta del Zotto MD PhD,

Department of Clinical and Experimental Sciences, Neurology Clinic, Brescia University Hospital, Italy

Andrea Morotti MD,

Department of Clinical and Experimental Sciences, Neurology Clinic, Brescia University Hospital, Italy

Loris Poli MD,

Department of Clinical and Experimental Sciences, Neurology Clinic, Brescia University Hospital, Italy

Maria Lorenza Muiesan MD,

Department of Clinical and Experimental Sciences, Clinica Medica, Brescia University Hospital, Italy

Massimo Salvetti MD,

Department of Clinical and Experimental Sciences, Clinica Medica, Brescia University Hospital, Italy

Enrico Agabiti Rosei MD,

Department of Clinical and Experimental Sciences, Clinica Medica, Brescia University Hospital, Italy

Silvia Lanfranconi MD, Department of Neurology, IRCCS Foundation Ca'Granda Hospital Policlinic Hospital, University of Milan, Italy

Pierluigi Baron MD PhD,

Department of Neurology, IRCCS Foundation Ca'Granda Hospital Policlinic Hospital, University of Milan, Italy 
Carlo Ferrarese MD PhD,

University of Milano Bicocca, San Gerardo Hospital, Monza, Italy

Emanuela Susani MD,

University of Milano Bicocca, San Gerardo Hospital, Monza, Italy

Giacomo Giacalone MD,

Milan Scientific Institute San Raffaele University Hospital, Italy

Stefano Paolucci MD PhD,

Department of Rehabilitation, Santa Lucia Hospital, Rome, Italy

Raffaele Palmirotta MD,

Department of Laboratory Medicine \& Advanced Biotechnologies, IRCCS San

Raffaele Pisana, Rome, Italy

Fiorella Guadagni MD,

Department of Laboratory Medicine \& Advanced Biotechnologies, IRCCS San

Raffaele Pisana, Rome, Italy

Maurizio Paciaroni MD PhD,

Stroke Unit and Division of Cardiovascular Medicine, University of Perugia Santa

Maria della Misericordia Hospital, Sant'Andrea delle Fratte, Perugia, Italy

Elena Ballabio MD,

Department of Cerebrovascular Diseases, and Emilio Ciusani, PhD, Laboratory of

Clinical Investigation, Fondazione IRCCS Istituto Neurologico Carlo Besta, Milano, Italy

Eugenio A. Parati MD,

Department of Cerebrovascular Diseases, and Emilio Ciusani, PhD, Laboratory of Clinical Investigation, Fondazione IRCCS Istituto Neurologico Carlo Besta, Milano, Italy

Felix Fluri MD,

Department of Neurology, Basel University Hospital, Switzerland

Florian Hatz MD,

Department of Neurology, Basel University Hospital, Switzerland

Dominique Gisler MD,

Department of Neurology, Basel University Hospital, Switzerland

Margareth Amort,

Department of Neurology, Basel University Hospital, Switzerland

Steve Bevan PhD,

Stroke and Dementia Research Centre, St George's University of London, UK

Tom James BSc,

Stroke and Dementia Research Centre, St George's University of London, UK

Sandra Olsson PhD, 
Institute of Neuroscience and Physiology, the Sahlgrenska Academy at University of Gothenburg, Gothenburg, Sweden

Lukas Holmegaard MD, Institute of Neuroscience and Physiology, the Sahlgrenska Academy at University of Gothenburg, Gothenburg, Sweden

Ayse Altintas MD PhD, Department of Neurology, University Hospital of Istanbul, Turkey

Juan José Martin MD, Department of Neurology, University Hospital Sanatorio Allende, Cordoba, Argentina

Steven Kittner MD MPH, Maryland Stroke Center, Department of Neurology, University of Maryland School of Medicine, Baltimore, Maryland, USA

Braxton Mitchell PhD, Maryland Stroke Center, Department of Neurology, University of Maryland School of Medicine, Baltimore, Maryland, USA

Colin Stine PhD, Maryland Stroke Center, Department of Neurology, University of Maryland School of Medicine, Baltimore, Maryland, USA

Jeff O'Connell PhD, Maryland Stroke Center, Department of Neurology, University of Maryland School of Medicine, Baltimore, Maryland, USA

Nicole Dueker PhD, Maryland Stroke Center, Department of Neurology, University of Maryland School of Medicine, Baltimore, Maryland, USA

Peter J. Koudstaal MD PhD, Department of Neurology, Erasmus MC University Medical Center, Rotterdam, the Netherlands

Lonneke M.L. de Lau MD PhD, Department of Neurology, Erasmus MC University Medical Center, Rotterdam, the Netherlands

Albert Hofman MD PhD, Department of Epidemiology Erasmus MC Rotterdam

Benjamin F Verhaaren MD MSc, Department of Epidemiology Erasmus MC Rotterdam

Ralf Weber MD, Department of Internal Medicine, Erasmus MC

Andre G Uitterlinden PhD, Department of Internal Medicine, Erasmus MC 
Joan Montaner MD PhD,

Laboratorio Neurovascular, Institut de Recerca, Hospital Vall d'Hebron, Barcelona, Spain

Maite Mendioroz MD PhD,

Laboratorio Neurovascular, Institut de Recerca, Hospital Vall d'Hebron, Barcelona, Spain

Sunaina Yadav MSc,

Imperial College Cerebrovascular Research Unit (ICCRU), Imperial College London, UK

Muhammad Saleem Khan BSc,

Imperial College Cerebrovascular Research Unit (ICCRU), Imperial College London, UK

Michael Wilder MD,

University of Utah, USA

Ewoud van Dijk MD PhD,

Imperial College Cerebrovascular Research Unit (ICCRU), Imperial College London, UK

Noortje Maaijwee MD, Imperial College Cerebrovascular Research Unit (ICCRU), Imperial College London, UK

Loes Rutten-Jacobs MSc,

Donders Institute for Brain, Cognition and Behaviour, Centre for Neuroscience, Department of Neurology, Radboud University Nijmegen Medical Centre, Nijmegen, The Netherlands

Jamie Kramer BA, Departments of Neurology and Public Health Sciences University of Virginia, Charlottesville, Virginia, USA

Shaneela Malik MD, Henry Ford Hospital, Detroit, Michigan, USA

Ralf Weber MD, Henry Ford Hospital, Detroit, Michigan, USA

Thomas G Brott MD,

Department of Neurology, Mayo Clinic, Jacksonville, Florida, USA

Robert D Brown Jr MD, Department of Neurology, Mayo Clinic, Rochester, Minnesota, USA

Andrew Singleton $\mathrm{PhD}$, Molecular Genetics Section, Laboratory of Neurogenetics, National Institute on Aging, NIH John Hardy PhD, 
Department of Molecular Neuroscience Institute of Neurology, University College London, London, United Kingdom

Stephen S Rich PhD,

Department of Public Health Sciences and the Center for Public Health Genomics, University of Virginia, Charlottesville, Virginia, USA

Christian Tanislav MD PhD,

Department of Neurology, University Hospital of Giessen, Germany

Jan Jungehülsing

Charité Universitätsmedizin Berlin, Department of Neurology, Berlin, Germany.

David Werring Prof,

UCL Queen Square Institute of Neurology, London, UK

Henry Houlden Prof,

UCL Queen Square Institute of Neurology, London, UK

Varinder Alg Mr,

UCL Queen Square Institute of Neurology, London, UK

Isabel Hostettler Dr,

UCL Queen Square Institute of Neurology, London, UK

Stephen Bonner Dr,

The James Cook University Hospital, Middlesbrough, UK

Daniel Walsh Mr,

King's College Hospital NHS Foundation Trust, London, UK

Diederik Bulters Mr,

University Hospital Southampton NHS Foundation Trust, Southampton UK

Neil Kitchen Mr,

The National Hospital of Neurology and Neurosurgery, London, UK

Martin Brown Prof,

UCL Queen Square Institute of Neurology, London, UK

Joan Grieve Ms,

The National Hospital of Neurology and Neurosurgery, London, UK

Joan Grieve Ms,

The National Hospital of Neurology and Neurosurgery, London, UK

Gareth Roberts Mr,

Royal Preston Hospital, UK

Timothy Jones Mr,

St George's Hospital, London, UK

Giles Critchley Mr,

Hurstwood Park Neurological Centre, UK 
Pankaj Sharma Prof,

Imperial Healthcare, Charing Cross Hospital, London, UK

Richard Nelson Mr,

Frenchay Hospital, Bristol, UK

Peter Whitfield Prof,

Derriford Hospital, Plymouth, UK

Stuart Ross Mr,

Leeds General Infirmary, UK

Hiren Patel Mr,

Salford Royal Hospital, UK

Paul Eldridge Mr,

The Walton Centre, Liverpool, UK

Kari Saastamoinen Dr,

The Royal London Hospital, London, UK

Umang Patel Mr,

Sheffield Teaching Hospitals NHS Trust, UK

Enas Lawrance Dr,

Mayday Hospital, Croydon, UK

Subha Vandabona Dr,

Mount Gould Hospital, Plymouth, UK

David Mendelow Professor,

Newcastle University Hopsitals NHS Trust

Rachel Teal Ms,

Oxford Radcliffe Infirmary, UK

Orlando Warner Dr,

Oxford Radcliffe Infirmary, UK

Peter Kirkpatrick Professor

Addenbrooke's Hospital, Cambridge, UK

Sudha Seshadri,

Department of Neurology,Boston University School of Medicine, Framingham Heart

Study, Boston, MA, USA

Laura Kilarski,

University of Cologne,Germany

Hyacinth I Hyacinth,

Aflac Cancer and Blood Disorder Center, Department of Pediatrics, Emory

University School of Medicine, Atlanta, GA, USA

Jamary Oliveira, 
Filho Stroke and Cardiomyopathy Clinics,Federal University of Bahia, Brazilian National Research Committee (CNPq), Instituto Nacional de Ciencia e Tecnologiaem Doencas Tropicais (INCT-DT),Salvador,Brazil

Sandro Marini, Center for Human Genetic Research,Harvard Medical School,Boston, MA,USA Sunaina Yadav, Imperial College Cerebrovascular Research Unit, Department of Clinical Neuroscience,Imperial College London,London,UK

Paul Nyquist, Medicine,Baltimore, MD,USA Department of Anesthesiology and Critical Care Medicine,Johns Hopkins

Cathryn Lewis, Department of Medical and Molecular Genetics, King's College London, SGDP Centre, Institute of Psychiatry, Psychology \& Neuroscience, King's College London,London,UK Bjorn Hansen, Department of Clinical Sciences Lund, Neurology,Lund University,Department of Neurology and Rehabilitation Medicine, Skåne University Hospital,Lund,Sweden

Bo Norrving,

Sahlgrenska Academy at University of Gothenburg, Lund University,Department of Neurology and Rehabilitation Medicine,Skåne University Hospital,Lund,Sweden

Gustav Smith,

Department of Clinical Sciences Lund, Cardiology,Lund University, The Section for Heart Failure and Valvular Disease, VO Heart and Lung medicine,Skane University Hospital,Lund,Sweden

Jonathan Rosand, Department of Neurology,Massachusetts General Hospital,Center for Human Genetic Research,Massachusetts General Hospital (MGH),Boston, MA,USA

Alessandro Biffi, Division of Behavioral Neurology, Department of Neurology,Massachusetts General Hospital,Center for Human Genetic Research,Massachusetts General Hospital (MGH),Boston, MA,USA

Christina Kourkoulis, Department of Neurology,Massachusetts General Hospital,Center for Human Genetic Research,Massachusetts General Hospital (MGH),Boston, MA,USA

Chris Anderson, Center for Genomic Medicine,Massachusetts General Hospital (MGH),J. Philip Kistler Stroke Research Center, Department of Neurology, Massachusetts General Hospital (MGH),Boston, MA,USA

Anne-Katrin Giese, Department of Neurology,Massachusetts General Hospital, Harvard Medical School, Department of Neurology,J. Philip Kistler Stroke Research Center, 
MGH,Boston, MA,USA Ralph Sacco,Department of Neurology,Miller School of Medicine, University of Miami,Miami, FL,USA

Pankaj Sharma, Institute of Cardiovascular Research, Royal Holloway University of London,London,UK

Oh Young Bang, Department of Neurology,Samsung Medical Center, Sungkyunkwan University School of Medicine, Seoul, Korea

Jong-Won Chung, Department of Neurology,Samsung Medical Center, Sungkyunkwan University School of Medicine, Seoul, USA

Gyeong-Moon Kim, Department of Neurology,Samsung Medical Center, Sungkyunkwan University School of Medicine, Seoul, Korea

Qishuai Zhuang, Department of Pharmacology,School of Life Science and Biopharmaceutical Sciences, Shenyang Pharmaceutical University, Shenyang,China

Wayne Sheu,

Department of Internal Medicine,Taichung Veterans General Hospital, Taichung,Taiwan Steven Lubitz,Department of Cardiology,Massachusetts General Hospital, Harvard Medical School,Boston, MA,USA

David Werring,

Stroke Research Centre, Department of Brain Repair and Rehabilitation,University College London Institute of Neurology,London,UK

John Hardy, Reta Lila Weston Institute, UCL Institute of Neurology,University College London,London,UK

June Smalley, Reta Lila Weston Institute, UCL Institute of Neurology,University College London,London,UK

Romain Bourcier, Department of Neuroradiology,University Hospital of Nantes,Nantes,France Joanna Howson, MRC/BHF Cardiovascular Epidemiology Unit, Department of Public Health and Primary Care,University of Cambridge,Cambridge,UK

Alessandra Granata,

Department of Clinical Neurosciences, University of Cambridge,Cambridge,UK Anna Drazyk,Department of Clinical Neurosciences, University of Cambridge,Cambridge,UK 
Hugh Markus,

Stroke Research Group, Division of Clinical Neurosciences,University of Cambridge, Cambridge,UK

Joanna Wardlaw,

Neuroimaging Sciences, University of Edinburgh, Centre for Clinical Brain

Sciences,University of Edinburgh, Edinburgh,UK

Braxton Mitchell,

Department of Medicine,University of Maryland School of Medicine, Geriatrics

Research and Education Clinical Center,Baltimore, Veterans Administration Medical

Center,Baltimore, MD,USA

John Cole,

Department of Neurology,University of Maryland School of Medicine and Baltimore, VAMC,Baltimore, MD,USA

Anbupalam Thalamuthu,

Centre for Healthy Brain Ageing, Psychiatry,University of New South Wales

(UNSW),Sydney,Australia

Jemma Hopewell,

Clinical Trial Service Unit and Epidemiological Studies Unit, Nuffield Department of

Population Health,University of Oxford,Oxford,UK

Robin Walters,

Clinical Trial Service Unit and Epidemiological Studies Unit, Nuffield Department of Population Health,University of Oxford,Oxford,UK

lain Turnbull,

Clinical Trial Service Unit and Epidemiological Studies Unit, Nuffield Department of Population Health,University of Oxford,Oxford,UK

Bradford Worrall,

Departments of Neurology and Public Health Sciences,University of Virginia School of Medicine,Charlottesville, VA,USA

Josh Bis,

Cardiovascular Health Research Unit, Department of Medicine,University of

Washington, Seattle, WA,USA

David Tirschwell,

Comprehensive Stroke Care at the UW Medicine Stroke Center at Harborview Medical Center,University of Washington, Seattle, WA,USA

Alex Reiner,

Department of Epidemiology,University of Washington, Fred Hutchinson Cancer

Research Center, University of Washington, Seattle, WA,USA

Raj Dhar, 
Department of Neurology,Washington University School of Medicine,St. Louis, MO,USA Laura Heitsch,Department of Neurology, Radiology, and Biomedical Engineering,Washington University School of Medicine,St. Louis, MO,USA

Jin-Moo Lee,

Department of Neurology, Radiology, and Biomedical Engineering, Washington University School of Medicine,St. Louis, MO,USA

Janne Mortenson, Department of Neurology,Aarhus University Hospital,Aarhus, Denmark

Sylvia Wassertheil-Smoller, Department of Epidemiology and Population Health,Albert Einstein College of Medicine,New York, NY,USA

Kameshwar Prasad,

Department of Neurology and Stroke Unit;and Director of Clinical Epidemiology,,All India Institute of Medical Sciences (AIIMS),New Delhi,India

Mark Fisher, Department of Neurology,University of Massachusetts Medical School, Worchester, MA,USA

Christopher Traenka,

Neurology Clinic,Basel University Hospital,Basel,Switzerland

Xingwu Wang,

Beijing Hypertension League Institute,Beijing,China

Yongjun Wang,

Center of Stroke,Beijing Insitute for Brain Disorders,Department of Neurology,

Beijing Tiantan Hospital, Capital Medical University,Beijing,China

Francois Rouanet,

Department of Neurology,Bordeaux University Hospital,Bordeaux,France

Igor Sibon,

Department of Neurology,Bordeaux University Hospital,Bordeaux,France

Chloé Sarnowski,

Department of Biostatistics,Boston University,Boston,USA

Pauline Maillard,

Department of Neurology,University of California at Davis,Sacramento, CA,USA

Aleksandra Pikula,Department of Neurology,University of Toronto,Toronto,USA

Hugo Javier Aparicio,

Department of Neurology,Boston University School of Medicine,Boston,USA Philip

Wolf,Boston University School of Medicine,Boston,USA

Josee Dupuis,

Boston University School of Public Health,Boston,USA

Qiong Yang, 
Boston University School of Public Health,Boston,USA

Gustavo Luvizutto,

Rehabilitation department,Botucatu Medical School,Botucatu School of

Medicine,University Estadual Paulista Júlio de Mesquita Filho, District of Rubião

Junior,Botucatu,Brazil

Daniel Chasman,

Division of Preventive Medicine,Brigham and Women's Hospital,Harvard Medical

School,Boston, MA,USA

Kathryn Rexrode,

Department of Medicine,Brigham and Women's Hospital,Harvard Medical

School,Boston,USA

Andrea Harriot,

Department of Neurology,Brigham and Women's Hospital, Massachusetts General Hospital,Boston, MA,USA

Chia-Ling Phuah,

Center for Human Genetic Research, Massachusetts General Hospital

(MGH),Division of Neurocritical Care and Emergency Neurology, Department of

Neurology,Boston, MA,USA

Gustavo Santo,

Stroke Unit,Centro Hospitalar e Universitário de Coimbra, CHUC,Coimbra,Portugal

Ales Tomek,Department of Neurology, 2nd Faculty of Medicine,Charles University in

Prague and Motol University Hospital,Prague,Czech Republic

Jen Gerard,

Department of Cardiology,Children's Hospital of Wisconsin,Milwaukee,USA Cara

Carty,Children's Research Institute,Children's National Medical Center,Center for

Translational Science,George Washington University, Washington,USA

Guiyou Liu,

Chinese Academy of Sciences (CAS),Beijing, China

Sanjith Aaron,

Department of Neurological Sciences,Christian Medical College,Vellore,India

Christhunesa S. Christudass,

Department of Neurological Sciences,Christian Medical College,Vellore,India

BSB Salomi,

Department of Neurological Sciences,Christian Medical College,Vellore,India

Dharambir Sanghera,

Department of Pediatrics, College of Medicine, University of Oklahoma Health

Sciences Center,Department of Pharmaceutical Sciences, College of Pharmacy, University of Oklahoma Health Sciences Center,Oklahoma City,USA

Amelia Boehme, 
Department of Neurology,Columbia University,New York,USA

Mitchell Elkind,

Neurological Institute of New York,Columbia University College,New York,USA

Gudmar Thorleifsson,deCODE genetics/AMGEN inc,Reykjavik,Iceland

Solveig Gretarsdottir,

deCODE genetics/AMGEN inc, Reykjavik,Iceland

Leslie Lange,

University of Colorado Denver Anschutz Medical Campus,Denver, CO,USA

Natalia Rost,

Department of Neurology, Massachusetts General Hospital, Harvard Medical

School, Boston, MA, USA,Department of Neurology, J. Philip Kistler Stroke

Research Center, MGH,Boston, MA,USA

Michael James,

Translational Acute Brain Injury Research Center,Duke University,Durham,USA

Jill Stewart,

Department of Neurology,Duke University School of Medicine,Durham,USA

Larry Goldstein,

Department of Medicine, Duke Stroke Center,Duke Univesrity School of

Medicine,Durham, NC, USA

Salina Waddy,

Cardiovascular and Stroke Genetics Working Group,Emory

University,Atlanta,USA Carla Ibrahim-Verbaas,Department of Neurology,Erasmus

MC, Rotterdam,Netherlands

Dina Vojinovic,

Department of Epidemiology,Erasmus MC, University Medical Center

Rotterdam,Clinic for Neurology and Psychiatry for Children and Youth,University of

Belgrade, Faculty of Medicine,Rotterdam,Netherlands

Arfan Ikram,

Department of Epidemiology,Erasmus University Medical

Center,Rotterdam,Netherlands Hieab Adams,Department of Epidemiology,Erasmus

University Medical Center,Rotterdam,Netherlands Fadi Charchar,L.E.W.

Carty Cardiovascular Genomics laboratory,Federation University

Australia,Ballarat,Australia

Vincent Thijs,

Stroke Division,Florey Institute of Neuroscience and Mental Health,Department of Neurology,Austin Health,Heidelberg,Australia

Eugenio Parati, Laboratory of Cellular Neurobiology,Fondazione IRCCS Istituto Neurologico C. Besta, Cerebrovascular Disease Unit,Cerebrovascular Disease Unit,Fondazione IRCCS Istituto Neurologico C Besta,Milan, Italy 
Giorgio Boncoraglio,

Department of Cerebrovascular Diseases,Fondazione IRCCS Istituto Neurologico

Carlo Besta,Milan,Italy

Charles Kooperberg,

Division of Public Health Sciences, Fred Hutchinson Cancer Research

Center,Seattle,USA

Sherrine Abboud,

Laboratory of Experimental Neurology,Free University of Brussels

(ULB),Brussels,Belgium

Ramin Zand,

Department of Neuroscience,Geisinger Health System,Danville,USA

Philippe Bijlenga,

Departement des neurosciences cliniques, Clinique de neurochirurgie,Geneva

University Hospital,Geneva,Switzerland

Magdy Selim,

Beth Israel Deaconess Medical Center,Harvard Medical School,Boston,USA

caspar Grond-Ginsbach,Department of Neurology,Heidelberg University

Hospital,Heidelberg,Germany

Olli Happola,

Department of Neurology,Helsinki University Central Hospital, Helsinki,Finland

Daniel Strbian,

Department of Neurology,Helsinki University Central Hospital, Clinical

Neurosciences, Neurology,University of Helsinki,Helsinki,Finland

Liisa Tomppo,

Department of Neurology,Helsinki University Central Hospital, Helsinki,Finland

Hanne Sallinen,Department of Neurology,Helsinki University Hospital,

Helsinki,Finland

Abhishek Pathak,

Department of Neurology,Heritage Hospital,Department of Neurology,Neuro Expert

Clinic,Varanasi,India

Dorothea Pfeiffer,

Department of Neurology,Hiedelberg University Hospital,Heidelberg,Germany Pablo

Bonardo,Department of Neurology,Hospital Británico

de Buenos Aires,

Ciudad Autónoma de Buenos Aires,Argentina

Joao Jose Freitas de Carvalho,

Hospital Geral de Fortaleza/SUS Fortaleza,Ceará,Brazil

Priscila Ribeiro,

Hospital Governador Celso Ramos,Florianópolis,Brazil 
Nuria Torres,

Stroke Pharmacogenomics and Genetics,Hospital Mútua de Terrassa, Fundació Docència i Recerca Mútua Terrassa,Terrassa,Spain

Miguel Barboza, Department of Neurology,Hospital Rafael A. Calderon Guardia,San Jose,Costa Rica Melanie Laarman,Cardiac Development and Genetics Group,Hubrecht Institute,Utrecht,Netherlands Roberta Carriero,Inflammation and immunology laboratory,Humanitas Clinical Institute,Pavia, Italy Elizabeth Holliday,Public Health Stream,Hunter Medical Research Institute,Faculty of Health and Medicine,University of Newcastle,New Lambton,Australia

Androniki Plomaritoglou, Neurology,Hygeia Hospital,Marousi,Greece

Johan Bjorkegren, Department of Genetic and Genomic Sciences, Icahn School of Medicine,New York,USA

Yu-Feng Yvonne Chan, Department of Genetic and Genomic Sciences, Icahn School of Medicine, Mount Sinai,New York,USA

Villi Gudnason, Icelandic Heart Association Research Institute,Faculty of Medicine,University of Iceland,Kopavogur, Iceland

Jordi Jimenez-Conde, Neurovascular Research Group (NEUVAS). Neurology Department., IMIM Hospital del Mar,Barcelona,Spain

Carolina Soriano, Department of Neurology, Neurovascular Research Group,IMIM-Hospital del Mar,Barcelona,Spain

Jaume Roquer, Neurology Service,IMIM-Hospital del Mar, Institut Hospital del Mar d'investigacions Mèdiques,Barcelona,Spain

Paul Bentley, Faculty of Medicine Department of Medicine, Imperial College London,London,UK Dipender Gill,Centre for Pharmacology and Therapeutics, Imperial College London, London,UK Ganesh Chauhan,Centre for Brain Research,Indian Institute of Science,Bangalore,India

Elisabeth Tournier-Lasserve, UMR-S 740,INSERM,Paris,France Sara Kaffashian,INSERM U1219 Bordeaux Population Health Research Center,Bordeaux,France Cecilia Samieri,INSERM U1219 Bordeaux Population Health Research Center,ISPED,University of Bordeaux,Bordeaux,France 
Carole Dufouil,

INSERM U1219 Bordeaux Population Health Research Center,Department of

Public Health, CHU Bordeaux,Bordeaux,France

Stephanie Debette,

INSERM U1219 Bordeaux Population Health Research Center, Bordeaux,

France,University of Bordeaux,France

Aniket Mishra,

INSERM U1219 Bordeaux Population Health Research Center, Bordeaux, France,University of Bordeaux,France

Lawrence Wee,

Data Analytics Department,Institute for Infocomm Research

(I2R),Singapore,Singapore

Saima Siddiqi,

Institute of Biomedical And Genetic Engineering,Islamabad,Pakistan

Jer-Yuarn Wu,

Institute of Biomedical Sciences, Academia Sinica, Taipei,Taiwan

Tai-Ming Ko,

Institute of Biomedical Sciences, Academia Sinica, Graduate Institute of Integrated

Medicine, China Medical University,Taipei,Taiwan

Silvia Bione,

Computational Biology Unit,Institute of Molecular Genetics-National Research

Council,Pavia,Italy

Katarina Jood,

Department of Clinical Neuroscience, Institute of Neuroscience and Physiology, Sahlgrenska Academy at University of Gothenburg, Gothenburg,Sweden

Turgut Tatlisumak, Department of Clinical Neuroscience, Institute of Neuroscience and Physiology, Sahlgrenska Academy at University of Gothenburg, Sahlgrenska University Hospital,Gothenburg,Sweden

Lukas Holmegaard,

Department of Clinical Neuroscience, Institute of Neuroscience and Physiology, Sahlgrenska Academy at University of Gothenburg, Gothenburg,Sweden

Antonio Arauz,

Stroke Clinic,Instituto Nacional de Neurologia y Neurocirugia, Manuel Velasco

Suarez,Mexico City,Mexico

Michal Korostynski,

Department of Molecular Neuropharmacology,Polish Academy of

Sciences, Warsaw,Poland

Lenore Launer, 
Laboratory of Epidemiology and Population Science, Intramural Research Program, National Institutes of Health, National Institute on Aging,Bethesda,USA

Suo Yue, Ion Torrent by Life Technologies (company),Carnegie Mellon University,San Francisco,USA

anna bersano, Cerebrovascular Unit,IRCCS Foundation C. Besta Neurological Institute,Milan,Italy Tomasz Dziedzic,Department of Neurology,Jagiellonian University,Krakow,Poland

Karol Józef Juchniewicz, Jagiellonian University,Krakow,Poland

Adamski Mateusz, Jagiellonian Centre for Experimental Therapeutics,Jagiellonian University, Krakow,Poland

Joanna Pera,

Department of Neurology,Jagiellonian University Medical College,Krakow,Poland Agnieszka Slowik,Department of Neurology,Jagiellonian University Medical College,Krakow,Poland Andrzej Urbanik, Department of Radiology,Jagiellonian University Medical College,Krakow,Poland Jerzy Gasowski,Department of Internal Medicine and Gerontology,Jagiellonian University Medical College,Krakow,Poland Rafa? Olszanecki,Faculty of Medicine,Jagiellonian University Medical College,Krakow,Poland

Marcin Wnuk, Jagiellonian University Medical College,Poland

Christopher Levi, John Hunter Hospital, Hunter Medical Research Institute and University of Newcastle,Newcastle,Australia

Aaron Gusdon, Department of Neurology,Johns Hopkins Medicine,University of Pittsburgh School of Medicine,Baltimore,USA

Konstantinos Kostulas, Department of Clinical Neuroscience, Karolinska Institute,Stockholm,Sweden Milita Crisby,Division of Clinical Geriatrics, Neurobiology Care Sciences and Society,Karolinska Institute,Huddinge,Sweden Lina Keller,Karolinska Institutet,Stockholm,Sweden Kristina Schlicht,Institut für Medizinische Informatik und Statistik,Kiel University,Kiel,Germany

Jessye Maxwell,

Social genetic and Developmental Psychiatry,King's College London,London, Christof Haffner,Institute for Stroke and Dementia Research,Klinikum der Universität München,Munich,Germany

Marco Duering, 
Institute for Stroke and Dementia Research,Klinikum der Universität München, Ludwig-Maximilians-University LMU,Munich,Germany

Jeremiasz Jagiella,

Krakow University Hospital,Krakow,Poland

Robin Lemmens,

Department of Neurosciences, Experimental Neurology,KU Leuven - University of Leuven,Department of Neurology,University Hospitals Leuven, VIB Center for Brain \& Disease Research, Leuven,Belgium

Jun Hata,

Department of Epidemiology and Public Health,Kyushu University, Graduate School of Medical Sciences,Fukuoka,Japan

Toshiharu Ninomiya,

Department of Epidemiology and Public Health,Kyushu University, Graduate School of Medical Sciences, Fukuoka,Japan

Vinh Nguyen,

School of Psychological Science,La Trobe University,Melbourne,Australia

Bjorn Logi Thorarinsson,

Department of Neurology,Landspítali Háskólasjúkrahús, Reykjavík,Iceland Saskia

Lesnik Oberstein,Department of Clinical Genetics,Leiden University Medical

Center,Leiden,Netherlands

Tsong-Hai Lee,

Neurology department and Stroke center, Linkou Chang Gung Memorial

Hospital,Taipei,Taiwan

Alexandr Rakitko,

Lomonosov Moscow State University,Moscow,Russia

Rainer Malik,

Institute for Stroke and Dementia Research,Ludwig-Maximilians-University, Klinikum der Universität München,Munich,Germany

Martin Dichgans,

Institute for Stroke and Dementia Research,Ludwig-Maximilians-University,

Klinikum der Universität München, Munich Cluster for Systems Neurology

(SyNergy),Munich,Germany

Arne Lindgren,

Department of Clinical Sciences Lund, Neurology,Lund University,Department of Neurology and Rehabilitation Medicine,Skåne University Hospital,Lund,Sweden

Johan Wasselius,

Department of Clinical Sciences Malmö,Lund University,Vascular Center,Skåne University Hospital,Malmö,Sweden

Mattias Drake, 
Diagnostic Radiology,Lund University,Lund,Sweden Olle Melander,Department of

Clinical Sciences,Lund University,Lund,sweden

Martin Stenman,

Geriatric Medicine,Lund University,Lund,Sweden

Andreea llinca,

Neurology,Lund University,Lund,Sweden

Julie Staals,

Department of Neurology,Maastricht University Medical Center (MUMC+),

Cardiovascular Research Institute Maastricht (CARIM),Maastricht,Netherlands

Ariane Sadr-Nabavi,

Medical Genetics Research Center (MGRC),Mashhad University of Medical

Sciences (MUMS), School of Medicine,Department of Medical Genetics,Mashhad

University of Medical Sciences (MUMS), Faculty of Medicine,Mashhad,Iran

Katherine Crawford,

Center for Genomic Medicine, Massachusetts General Hospital,Boston, MA,USA

Jose Florez,Center for Human Genetic Research, MGH,Massachusetts General

Hospital, Harvard Medical School, Boston, MA,USA

Umme Lena,

Massachusetts General Hospital,South Hadley, MA,USA

Farrah Mateen,

Department of Neurology,Massachusetts General Hospital,Boston, MA,USA

Hakan Ay,

Department of Neurology,Massachusetts General Hospital,Boston, MA,USA

Ona Wu,

Department of Neurology, JPK Stroke Research Center,Massachusetts General

Hospital and Harvard Medical School, Department of Radiology, Athinoula A

Martinos Center for Biomedical Imaging, MGH,Boston, MA,USA

Markus Schirmer,

Department of Neurology, J. Philip Kistler Stroke Research Centre,Massachusetts General Hospital and Harvard Medical School,Boston,USA

Javier Romero,

Division of Neurocritical Care and Emergency Neurology, Department of

Neurology,Massachusetts General Hospital, Harvard Medical School,J. P. Kistler

Stroke Research Center,Massachusetts General Hospital, Harvard Medical

School,Boston, MA,USA

Steve Cramer,

Neurology, Anatomy \& Neurobiology, and Physical Medicine \&

Rehabilitation,University of California Irvine,Irvine,USA

Polina Golland, 
The Computer Science and Artificial Intelligence Laboratory, Massachusetts Institute of Technology,Cambridge,USA

Bertram Mueller,

Myhsok Max-Planck-Institute of Psychiatry,Munich,Germany

Robert Brown,

Department of Neurology,Mayo Clinic,Rochester, MN,USA

James Meschia,

Department of Neurology,Mayo Clinic,Jacksonville,USA

Owen A. Ross,

Department of Medical Genetics and Neuroscience,Mayo Clinic,Jacksonville,USA

Thomas Brott,Neurology,Mayo Clinic, Department of Surgery, University of Medicine and Dentistry of New Jersey,Jacksonville, FL,USA

Guillaume Pare,

Population Health Research Institute,McMaster University,Hamilton,Canada

Mike Chong,

Population Health Research Institute,McMaster University,Hamilton,Canada

Ossama yassin mansour,

Department of Neurology,Medical school of Alexandria,Alexandria

Governorate,Egypt

Bartosz Karaszewski,

Department of Adult Neurology,Medical University of Gdansk \& University Clinical

Centre in Gdansk,Gdansk,Poland

Christian Enzinger,

Department of Neurology,Medical University of Graz,Graz,Austria Helena

Schmidt,Institute of Molecular Biology and Biochemistry,Medical University of

Graz,Graz,Austria

Reinhold Schmidt,

Department of Neurology,Medical University of Graz,Graz,Austria

Stephan Seiler,

Department of Neurology,Medical University of Graz,Graz,Austria

Alexander Pichler,

Department of Neurology,Medical University of Graz,Graz,Germany

Bruce Ovbiagele,

Department of Neurology,Medical University of South Carolina,Charleston,USA

Yoshiji Yamada,

Department of Human Genomics,Mie University, Life Scinece Research

Center,Mie,Japan

Tatjana Rundek, 
Department of Neurology,Miller School of Medicine, University of Miami,Miami, FL,USA

Susan Blanton, Hussman Institute for Human Genomics,Miller School of Medicine, University of Miami,Miami, FL,USA

John $\mathrm{P}$, Hussman Institute for Human Genomics,Miller School of Medicine, University of Miami,Miami, FL,USA

Joseph Chern,

Department of Neurology, College of Medicine,National Cheng Kung

University,Tainan,Taiwan

Chris O'Donnell,

Cardiovascular Epidemiology and Human Genomics Branch,National Heart, Lung and Blood Institute, Intramural Research,Veterans Administration Healthcare,Center for Population Genomics,Boston, MA,USA

Roderick Corriveau, National Institute of Neurological Disorders and Stroke,National Institute of Health, University of California,Bethesda, MD,USA

Pallab Bhattacharya,

National Institute of Pharmaceutical Education and Research,Ahmedabad,India Andrew Singleton,Laboratory of Neurogenetics,National Institute on Aging, Intramural Research Program,Bethesda, MD,USA

Katrina Gwinn, National Institute of Neurological Disorders and Stroke,National Institutes of Health,Bethesda, MD,USA

BHARATENDU CHANDRA, General neurology and stroke,National University Hospital,Singapore,Singapore Christopher Chen, Department of Pharmacology,National University of Singapore,Singapore,Singapore

Raj Kalaria, Institute of Neuroscience,Newcastle University,Newcastle, UK

Jim Koenig,

Division of Neuroscience, Stroke Program in the Neural Environment, NINDS,Bethesda, MA,USA

Om Prakash Singh, Department of Environmental Studies,North Eastern Hill University (NEHU),Shillong,India

Akintomi Olugbodi, 
Obafemi Awolowo University,lle-Ife,Nigeria Bishwa Sapkota,Harold Hamm Diabetes Center,Oklahoma University,Oklahoma City,USA Rebecca Jackson,Center for women's health, Ohio State University Wexner Medical Center,Colombus,USA

Eva Giralt,

Parc de Salut Mar,Barcelona,Spain

Danish Saleheen,

Department of Genetics,Perelman School of Medicine, University of Pennsylvania,Pennsylvania, PA,USA

Frank-Erik de Leeuw, Department of Neurology,Radboud University Medical

Center,Nijmegen,Netherlands

Karin Klijn,

Department of Neurology,Radboud University Medical Centre, Donders Institute for Brain, Cognition and Behaviour,Department of Neurology and Neurosurgery,University Medical Center, Brain Center Rudolf Magnus,Nijmegen,Netherlands

Jes Olesen,

Department of Clinical Medicine,Rigshospitalet - Neurocentret,Glostrup,Denmark

Yoichiro Kamatani,

Laboratory for Statistical Analysis, RIKEN Center for Integrative Medical

Sciences, Center for Genomic Medicine, Kyoto University Graduate School of

Medicine,Yokohama,Japan

Michiaki Kubo,

RIKEN Center for Integrative Medical Sciences, Yokohama,Japan Yukinori

Okada,Laboratory for Statistical Analysis,RIKEN Center for Integrative Medical

Sciences,Department of Statistical Genetics, Osaka University Graduate School of Medicine,Yokohama,Japan

David Spence,

Stroke Prevention \& Atherosclerosis Research Centre,Robarts Research,London (ON), Canada

Annie Pedersen,

Västra Vötalandsregionen,Sahlgrenska Academy at University of Gothenburg,Gothenburg,Sweden

Maja Olsson, Department of Medical and Clinical Genetics, Institute of Biomedicine,Sahlgrenska Academy at University of Gothenburg, Gothenburg,Sweden

Turgut Tatlisumak, Department of Clinical Neurosciences/Neurology,Sahlgrenska Academy at University of Gothenburg, Institute of Neuroscience and Physiology,Sahlgrenska University Hospital,Gothenburg,Sweden 
Giacomo Giacalone,

Neurology Department,San Raffaele Scientific Institute,University Vita-Salute San Raffaele,Milan,Italy

Juan José Martín,

Department of Neurology,Sanatorio Allende,Cordoba,Argentina Rodrigo

Bazan,Department of Neurology,São Paulo State University (Unesp) Botucatu

Medical School,Botucatu,Brazil

Gabriel Braga,

Hospital das Clínicas,São Paulo State University (Unesp) Botucatu Medical

School,Botucatu,Brazil

Steve Bevan,

School of Life Science, University of Lincoln,Lincoln,UK

Huichun Xu,

Medicine department,School of Medicine, University of Maryland,Baltimore,USA

Tim Assimes,

Cardiovascular Medicine,School of Medicine, University of Stanford,Stanford,USA Jamie Wright, Anderson Cancer Center, MD Anderson Clinical Cancer

Genetics,School of Medicine, University of Texas,Houston,USA

Anton Raskurazhev,

Scientific Center of Neurology,Moscow,Russia

Wei Ling Lee,

National Neuroscience Institute,Singapore General Hospital,Department of Pediatrics,National University of Singapore, Yong Loo Lin School of

Medicine,Singapore,Singapore Eng King Tan,Department of Neurology,Singapore General Hospital, National Neuroscience Institute,Neuroscience and Behavioral Disorders Program,Duke-National University of Singapore Graduate Medical School,Singapore,Singapore

Philippe Burri,

Department of Cardiology,Skane University Hospital,Malmö,Sweden

Petrea Frid,

Critical Care Neurology,Skåne University Hospital, Lund university,Malmö,Sweden Christian Opherk,Department of Neurology,SLK-Kliniken

Heilbronn $\mathrm{GmbH}$, Heilbronn,Germany Chaeyoung Lee,Department of Bioinformatics and Life Science,Soongsil University,Seoul,Korea David Tregouet,Team Genomics and Pathophysiology of Cardiovascular Diseases,Sorbonne Universités, UPMC Univ Paris 06, INSERM, UMR_S 1166,ICAN Institute for Cardiometabolism and Nutrition,Paris,France

Zhen Deng, 
Department of Neurology,Southern Medical University, FIMMU,Guangzhou,China Hung Yi Chiou,School of Public Health, College of Public Health and Nutrition,Taipei Medical University, Taipei,Taiwan

Mahdi Habibi-koolaee, Department of Health Information Management,Tehran University of Medical Sciences (TUMS), Tehr?n,Iran

Murali Vijayan,

Garrison Institute on Aging,Texas Tech University Health Sciences

Center,Lubbock,USA

Thomas Leung,

Division of Neurology, Department of Medicine and Therapeutics, The Chinese University of Hong Kong,Hong-Kong,Hong-Kong

Lawrence Wong,

Division of Neurology, Department of Medicine and Therapeutics, The Chinese University of Hong Kong,Hong-Kong,Hong-Kong

Vincent Mok,

Department of Medicine and Therapeutics, The Chinese University of Hong Kong,Hong- Kong,Hong-Kong

Richard Choy, Department of Obstetrics and Gynecology, The Chinese University of Hong Kong,Hong-Kong,Hong-Kong

Christina Jern,

Department of Pathology and Genetics, The Sahlgrenska Academy at University of Gothenburg, Institute of Biomedicine,Gothenburg,Sweden

Elena Lebedeva,

Department of Neurology and Neurosurgery, The Urals State Medical

University,International Headache Center 'Europe-Asia', Yekaterinburg,Russia

Martin Farrall,

Department of Cardiovascular Medicine, The Wellcome Trust Centre for Human Genetics,Oxford,UK

Xu Jiayuan,

Department of radiology,Tianjin Medical University (TIJMU), Tianjin,China

Keat Wei Loo,

Tunku Abdul Rahman University, UTAR,Kampar Perak,Malaysia

Isabel Hostettler, Stroke Research Center,UCL Institue of Neurology,London,UK

Gabriel Rinkel, Department of Neurology and Neurosurgery,UMC Utrecht, Brain Center Rudolf Magnus, 
Utrecht,Netherlands

Anderson Goncalves,

Universidade da Região de Joinville,Joinville,Brazil

Paulo Franca,

Department of Medicine,Universidade da Região de Joinville -

Univille,Joinville,Brazil Norberto Cabral, Department of Medicine,Universidade da

Região de Joinville (Univille), Joinville,Brazil

Iscia Cendes,

Molecular Genetics Laboratory,Universidade Estadual de Campinas, Faculdade de Ciências Médicas,Campinas,Brazil

Caty Carrera,

Neurovascular Research Laboratory, Vall d'Hebron Institut of Research, Neurology and Medicine Departments,Universitat Autònoma de Barcelona, Vall d'Hebrón Hospital,Stroke Pharmacogenomics and Genetics,Fundacio Docència i Recerca Mutua Terrassa,Spain

Israel Fernandez-Cadenas, Neurovascular Research Laboratory, Vall d'Hebron Institut of Research, Neurology and Medicine Departments,Universitat Autònoma de Barcelona, Vall d'Hebrón Hospital,Stroke Pharmacogenomics and Genetics,Fundacio Docència i Recerca Mutua Terrassa,Barcelona,Spain

Joan Montaner, Neurovascular Research Laboratory,Universitat Autònoma de Barcelona, Vall d'Hebron Institute of Research,Neurology Department,Hospital Vall d'Hebron,Barcelona,Spain

Helen Kim, Institute for Human Genetics, The Center for Cerebrovascular Research (CCR),Université de Californie,San Francisco,USA

Arndt Rolfs,

Albrecht Kossel Institute,University Clinic of Rostock,Rostock,Germany

Mayowa Owolabi, Department of Neurology,University College Hospital,Ibadan,Nigeria Reecha Sofat,Metabolism \& Experi Therapeutics, Div of Medicine, Faculty of Medical Sciences,University College London,London,UK

Mark Bakker, University Medical Center Utrecht,Utrecht,Netherlands Dominique PV de Kleijn,Laboratory of Experimental Cardiology,University Medical Center Utrecht,ICIN-Netherlands Heart Institute,Utrecht,Netherlands

Ynte Ruigrok, 
Department of Neurology and Neurosurgery,University Medical Center Utrecht, Brain Center Rudolf Magnus, The Dutch Parelsnoer Institute-Cerebrovascular accident (CVA) Study Group,Utrecht,Netherlands

Allard Hauer,

Department of Neurology and Neurosurgery,University Medical Center Utrecht, Brain Center Rudolf Magnus,Utrecht,Netherlands

Sara L. Pulit,

Department of Neurology,University Medical Center Utrecht, Brain Center

Rudolf Magnus,

Utrecht,Netherlands

Ale Algra,

Department of Neurology and Neurosurgery,University Medical Center Utrecht, Brain Center Rudolf Magnus, Julius Center for Health Sciences and Primary

Care,University Medical Center Utrecht,Utrecht,Netherlands

Sander W. van der Laan,

Laboratory of Experimental Cardiology,University Medical Center Utrecht, Division

of Heart and Lungs, Utrecht,Netherlands

Mary Macleod,

Dept of Medicine \& Therapeutics,University of Aberdeen, King's

College,Aberdeen,UK

George Howard,

School of Public Health,University of Alabama,Birmingham, AL,USA

Hemant Tiwari,

Department of Biostatistics,University of Alabama,Birmingham, AL,USA

Ryan Irvin,

Department of Epidemiology,University of Alabama at Birmingham,Birmingham, AL,USA

Karen C. Albright,

Neurology,University Of Alabama Hospital, Spectrum Health Butterworth

Campus,Birmingham, AL,USA

Rodney Perry,

Department of Epidemiology,University of Alabama School of Medicine,Birmingham, AL,USA

Chelsea Kidwell, Department of Neurology,University of Arizona,Stroke Center at Georgetown,University of Georgetown,Tucson, AZ,USA

Aleksandra Pavlovic, Neurology Clinic, Clinical Center of Serbia,University of Belgrade, Faculty of Medicine,Belgrade, Serbia 
Christophe Tzourio,

University of Bordeaux,INSERM U1219,Bordeaux,France

Murali Sargurupremraj,

INSERM U1219 Bordeaux Population Health Research Center, University of

Bordeaux,Bordeaux,France

Sabrina Schilling,

University of Bordeaux,Bordeaux, Alessandro Padovani,Department of Clinical and Experimental Sciences, University of Brescia,Brescia,Italy

Alessandro Pezzini,

Department of Clinical and Experimental Sciences,University of Brescia, Neurology

Clinic,Brescia,Italy

Foad Abd-Allah,

Department of Neurology,University of Cairo,Cairo,Egypt

Charles DeCarli,

Alzheimer's Disease Center,University of Californa Davis,Sacramento, CA, USA

Yuqi Zhao,Department of Integrative Biology and Physiology,University of California, Los Angeles, CA,USA

David Liebeskind,

Department of Neurology,University of California, Los Angeles, CA,USA

Matthew Traylor,

Stroke Research Group, Division of Clinical Neurosciences, University of

Cambridge,Cambridge,UK

Rhea Tan,

Department of Clinical Neurosciences,University of Cambridge,Cambridge,England

John Danesh,

MRC/BHF Cardiovascular Epidemiology Unit, Department of Public Health and

Primary Care,, University of Cambridge, The National Institute for Health Research

Blood and Transplant Research Unit in Donor Health and Genomics, Wellcome

Trust Sanger Institute,Cambridge,UK

Susanna C. Larsson,

Department of Clinical Neurosciences, Neurology Unit,University of

Cambridge,Cambridge,UK

Loes Rutten,

Jacobs,Stroke Research Group,University of Cambridge, Division of Clinical

Neurosciences,DZNE,Cambdrige,UK

Amanda Donatti,

School of Medical Sciences, FCM,University of Campinas, UNICAMP,São

Paulo,Brazil

Wagner Avelar, 
Department of Neurology,University of Campinas, UNICAMP,São Paulo,Brazil Joseph Broderick, Gardner Neuroscience Institute, Comprehensive Stroke Center,University of Cincinnati,Cincinnati, OH,USA

Daniel Woo, University of Cincinnati College of Medicine,Cincinnati, $\mathrm{OH}, \mathrm{USA}$

Brett Kissela, Department of Neurology and Rehabilitation Medicine,University of Cincinnati Gardner Neuroscience Institute, Comprehensive Stroke Center,Cincinnati, $\mathrm{OH}, \mathrm{USA}$ Laura Garcia Ibenez, Institute for Cancer Genetics,University of Columbia,New York,USA

Rustam Salman,

Centre for Clinical Brain Sciences, University of Edinburgh, Edinburgh,UK

Cathie Sudlow,

Centre for Medical Informatics, Usher Institute, University of Edinburgh,

Edinburgh,UK

Kristiina Rannikmäe,

Centre for Medical Informatics, Usher Institute,University of

Edinburgh,Edinburgh,UK

Caitrin Wheeler McDonough,

College of Pharmacy,University of Florida,Gainesville, FL,USA

Scott Silliman,

Department of Neurology,University of Florida College of Medicine,Jacksonville, FL,USA

Oyunbileg Magvanjav, Department of Pharmacotherapy and Translational Research and Center for Pharmacogenomics, University of Florida, College of Pharmacy,Gainesville, FL,Japan USA

Tom van Agtmael, Insittute of Cardiovascular and Medical Sciences,University of Glasgow,Glasgow,UK Matthew Walters, School of Medicine, Dentistry and Nursing,University of Glasgow,Glasgow,UK Martin Söderholm,Bioinformatics Core Facility,University of Gothenburg,Gothenburg,Sweden

Erik Lorentzen, Bioinformatics Core Facility,University of Gothenburg,Gothenburg,Sweden

Sandra Olsson, University of Gothenburg,Gothenburg,Sweden

Tara Stanne, 
University of Gothenburg,Gothenburg,Sweden

Martina Olsson,

University of Gothenburg,Gothenburg,Sweden

Kazuma Nakagawa,

University of Hawaii, John A. Burns School of Medicine,Department of

Neurology,The Queen's Medical Center,Honolulu,USA

Rufus Akinyemi,

Institute for Advanced Medical Research and Training,University of Ibadan, College

of Medicine, Ibadan,Nigeria

loana Cotlatciuc,

Institute of Cardiovascular Research Royal Holloway,University of London,

London,UK

Jeff O'Connell,

Medicine department,University of Maryland,Animal Genetics Improvement

Laboratory,United States Department of Agriculture,Baltimore, MD,USA

Mary Sparks,

Department of Neurology,University of Maryland,Baltimore, MD,USA

John Sorkin,

Department of Neural and Pain Sciences, University of Maryland,Baltimore, MD,USA

Patrick McArdle,Division of Endocrinology, Diabetes and Nutrition,University of

Maryland School of Medicine,Baltimore, MD,USA

Tushar Dave,

Bioinformatics, University of Maryland School of Medicine,Baltimore, MD,USA

Colin Stine,

Epidemiology \& Public Health,University of Maryland School of Medicine,Baltimore, MD,USA

Steven Kittner,

Department of Neurology,University of Maryland School of Medicine and Baltimore, VAMC, MD, USA,Baltimore, MD,USA

Jill Naylor,

University of Melbourne,Melbourne,Australia

Devin Brown,

Department of Neurovascular,University of Michigan Medical School,Ann Arbor,USA Mario Di Napoli,Department of Neurology,University of Naples,Naples,Italy

Rose Du,

Comprehensive Cancer Center,University of New Mexico, Albuquerque, NM,USA

Tobias B. Kulik,

Neurology,University of New Mexico,Albuquerque, NM, USA

John Attia, 
School of Medicine and Public Health,University of Newcastle,New Lambton,Australia Shahbaz Zamani,University of Newcastle,Newcastle,Australia James E Faber,

Cell Biology and Physiology,University of North Carolina,Chapel Hill,USA

Peter Rothwell, Nuffield Department of Clinical Neurosciences,University of Oxford,Oxford,UK

Iona Millwood, Nuttfield Department of Population Health,University of Oxford,Oxford,UK

Elsa Valdés Márquez, CTSU-Clinical Trial Service Unit and Epidemiological Studies Unit,University of Oxford,Oxford,UK

Michelangelo Mancuso, Department of Neurology,University of Pisa,Pisa, Italy

Doralina Brum Souza, Department of Neurology,University of Sao Paulo,Ribeirao Preto,Brazil

Ranil de Silva,

Genetic, Diagnostic and Research Laboratory, Department of Anatomy,University of Sri Jayewardenepura, Faculty of Medical Sciences,Nugegoda,Sri Lanka

Riina Vibo,

Department of Neurology and Neurosurgery,University of Tartu,Tartu,Estonia Janika Korv, Department of Neurology and Neurosurgery,University of Tartu,Tartu,Estonia Jane Maguire, Faculty of Health,University of Technology Sydney,Ultimo,Australia

Myriam Fornage,

Brown Foundation Institute of Molecular Medicine // Human Genetics Center,University of Texas Health Science Center at Houston,Houston, TX,USA

Kachikwu Illoh, University of Texas Medical School,Houston, TX,USA

Dianna Milewicz, Division of Medical Genetics,University of TexasHealth Science Center, McGovern Medical School,Department of Internal Medicine,University of TexasHealth Science Center, McGovern Medical School,Houston, TX,USA

Jennifer Majersik, Neurology,University of Utah,Salt Lake City, UT,USA

Adam DeHavenon, Department of Neurology,University of Utah,Salt Lake City, UT,USA

Yashar Kalani, 
Neurobiology and Anatomy, University of Utah School of Medicine,Salt Lake City, UT,USA

Matthew Alexander,

Radiology and Imaging Sciences, University of Utah School of

Medicine,Neurosurgery, University of Utah School of Medicine,Salt Lake City, UT,USA

Mary Cushman,

Department of Hematology and oncology,University of Vermont, Medical

Center,Colchester,USA

Michele Sale,

Center for Public Health Genomics,University of Virginia,Charlottesville,

VA,USA Andrew Southerland, Departments of Neurology and Public Health

Sciences,University of Virginia,Charlottesville, VA,USA

Debra Owens,

Newborn Intensive Care Units,University of Virginia Children's

Hospital,Charlottesville, VA,USA

Keith Keene,

Center for Public Health Genomics,University of Virginia School of

Medicine,Charlottesville, VA,USA

Stephe Rich,

Center for Public Health Genomics, University of Virginia School of

Medicine,Charlottesville, VA,USA

Bruce Psaty,

Cardiovascular Health Research Unit, Department of Medicine,University of

Washington,Department of Epidemiology,University of Washington,Seattle, WA,USA

Will Longstreth,

Department of Epidemiology,University of Washington,Department of

Neurology,University of Washington,Seattle,USA

Masharip Atadzhanov,

Department of Neurology,University Teaching Hospital,Lusaka,Zambia

Stacey Quintero Wolfe,

Wake Forest Baptist Health,Winston-Salem, NC, USA

Carl Langefeld,

Center for Public Health Genomics and Department of Biostatistical Sciences, Wake Forest School of Medicine,Winston-Salem, NC,USA

Cheryl Bushnell,

Department of Neurology,Wake Forest University Medical Center,Winston-Salem, NC,USA

Carlos Cruchaga, 
Department of Psychiatry, The Hope Center Program on Protein Aggregation and Neurodegeneration (HPAN),Washington University School of Medicine,Department of Developmental Biology, Washington University School of Medicine,St. Louis, MO,USA

Jan Konrad,

Department of Psychiatry, The Hope Center Program on Protein Aggregation and Neurodegeneration (HPAN), Washington University, School of Medicine,St. Louis, MO,USA

Junfeng Liu,

West China Medical School, Sichuan University,Sichuan,China

Kevin Sheth,

Department of Neurology, Neuro Intensive Care \& Neurological Emergencies, Yale School of Medicine,Nw Haven, CT,USA

Guido Falcone,

Department of Neurology, Yale University School of Medicine, Program in Medical and Population Genetics, The Broad Institute of Harvard and MIT,New Haven, CT, USA

Kathleen Donahue J

Philip Kistler Stroke Research Center,Massachusetts General Hospital,USA

\section{Affiliations}

Department of Public Policy, Institute of Medical Science, The University of Tokyo, Tokyo, Japan.

Department of Public Policy, Institute of Medical Science, The University of Tokyo, Tokyo, Japan.

Laboratory of Genome Technology, Human Genome Center, Institute of Medical Science, The University of Tokyo, Tokyo, Japan. Laboratory of Clinical Genome Sequencing, Graduate School of Frontier Sciences, The University of Tokyo, Tokyo, Japan.

Laboratory of Genome Technology, Human Genome Center, Institute of Medical Science, The University of Tokyo, Tokyo, Japan.

Division of Molecular Pathology, Institute of Medical Science, The University of Tokyo, Tokyo, Japan.

The BioBank Japan, Tokyo, Japan.

Laboratory of Complex Trait Genomics, Graduate School of Frontier Sciences, The University of Tokyo, Tokyo, Japan. Laboratory for Statistical and Translational Genetics, RIKEN Center for Integrative Medical Sciences, Yokohama, Japan.

Department of Public Policy, Institute of Medical Science, The University of Tokyo, Tokyo, Japan. 
Division of Molecular Pathology, Institute of Medical Science, The University of Tokyo, Tokyo, Japan.

Aso lizuka Hospital, Fukuoka, Japan.

Department of Gastroenterology, Cancer Institute Hospital, Japanese Foundation for Cancer Research, Tokyo, Japan.

Department of Genetic Diagnosis, The Cancer Institute, Japanese Foundation for Cancer Research, Tokyo, Japan.

Fukujuji Hospital, Tokyo, Japan.

Department of Urology, Iwate Medical University School of Medicine, Iwate, Japan.

Department of Urology, Iwate Medical University School of Medicine, Iwate, Japan.

Department of Internal Medicine and Rheumatology, Juntendo University Graduate School of Medicine, Tokyo, Japan.

Department of Respiratory Medicine, Juntendo University Graduate School of Medicine, Tokyo, Japan.

Division of Pharmacology, Department of Biomedical Science, Nihon University School of Medicine, Tokyo, Japan. Division of Genomic Epidemiology and Clinical Trials, Clinical Ttrials Research Center, Nihon University. School of Medicine, Tokyo, Japan.

Division of Genomic Epidemiology and Clinical Trials, Clinical Ttrials Research Center, Nihon University School of Medicine, Tokyo, Japan.

Department of Bioregulation, Nippon Medical School, Tokyo, Japan.

Department of Hematology, Nippon Medical School, Tokyo, Japan.

National Hospital Organization Osaka National Hospital, Osaka, Japan.

Osaka International Cancer Institute (formerly, Osaka Medical Cancer Center for Cancer \& Cardiovascular Diseases), Osaka, Japan

Osaka International Cancer Institute (formerly, Osaka Medical Cancer Center for Cancer \& Cardiovascular Diseases), Osaka, Japan

Center for Clinical Research and Advanced Medicine, Shiga University of Medical Science, Shiga, Japan.

Tokushukai Group, Tokyo, Japan.

Tokushukai Group, Tokyo, Japan.

Department of Neurology and Neuropathology (the Brain Bank for Aging Research), Tokyo Metropolitan Geriatric Hospital and Institute of Gerontology, Tokyo, Japan.

Division of Clinical Genome Research, Institute of Medical Science, The University of Tokyo, Tokyo, Japan. 
Division of Genetics, The Institute of Medical Science, The University of Tokyo, Minato-ku, Tokyo, Japan.

Romain Bourcier, Richard Redon, Gervaise Loirand, Jean-Jacques Schott, Stéphanie Chatel, Emmanuelle Bourcereau, Christian Dina, Floriane Simonet, Eric Charpentier, Estelle Baron, Stéphanie Bonnaud, Benjamin Daumas-Duport, Bertrand Isidor, Jérôme Connault, Pierre Lebranchu, Thierry Le Tourneau University Hospital of Nantes, Nantes, France

University Hospital of Rouen, Rouen, France

Hocine Redjem, Mikael Mazighi, Jean Philippe Desilles - Fondation Rotschild Hospital, Paris, France

Saint Anne Hospital, Paris, France

Saint Anne Hospital, Paris, France

Saint Anne Hospital, Paris, France

Saint Anne Hospital, Paris, France

Saint Anne Hospital, Paris, France

Saint Anne Hospital, Paris, France

University Hospital of Limoges, Limoges, France

University Hospital of Limoges, Limoges, France

University Hospital of Limoges, Limoges, France

Clairval Hospital, Marseille, France

Clairval Hospital, Marseille, France

Clairval Hospital, Marseille, France

University Hospital of Angers, Angers, France

University Hospital of Angers, Angers, France

University Hospital of La Réunion, Saint-Denis de La Réunion, France

University Hospital of La Réunion, Saint-Denis de La Réunion, France

University Hospital of La Réunion, Saint-Denis de La Réunion, France

University Hospital of Amiens, Amiens, France

University Hospital of Montpellier, Montpellier, France

University Hospital of Montpellier, Montpellier, France

University Hospital of Montpellier, Montpellier, France

University Hospital of Toulouse, Toulouse, France

University Hospital of Toulouse, Toulouse, France 
University Hospital of Toulouse, Toulouse, France

University Hospital of Toulouse, Toulouse, France

University Hospital of Toulouse, Toulouse, France

University Hospital of Toulouse, Toulouse, France

University Hospital of Besançon, Besançon, France

University Hospital of Besançon, Besançon, France

University Hospital of Rennes, Rennes, France

University Hospital of Rennes, Rennes, France

University Hospital of Rennes, Rennes, France

University Hospital of Rennes, Rennes, France

University Hospital of Brest, Brest, France

University Hospital of Brest, Brest, France

University Hospital of Nancy, Nancy, France

University Hospital of Nancy, Nancy, France

University Hospital of Nancy, Nancy, France

University Hospital of Nancy, Nancy, France

University Hospital of Nancy, Nancy, France

Le Kremlin Bicêtre Hospital, Paris, France

Le Kremlin Bicêtre Hospital, Paris, France

Le Kremlin Bicêtre Hospital, Paris, France

Le Kremlin Bicêtre Hospital, Paris, France

Le Kremlin Bicêtre Hospital, Paris, France

Le Kremlin Bicêtre Hospital, Paris, France

Le Kremlin Bicêtre Hospital, Paris, France

University Hospital of Clermont-Ferrand, Clermont-Ferrand, France

University Hospital of Brdeaux, Bordeaux, France

University Hospital of Brdeaux, Bordeaux, France

University Hospital of Brdeaux, Bordeaux, France

University Hospital of Brdeaux, Bordeaux, France

La Pitié Salpétrière Hospital, Paris, France

La Pitié Salpétrière Hospital, Paris, France

University Hospital of Saint Etienne, Saint Etienne, France 
University Hospital of Dijon, Dijon, France

University Hospital of Dijon, Dijon, France

University Hospital of Dijon, Dijon, France

Colmar Hospital, Colmar, France

Colmar Hospital, Colmar, France

University Hospital of Tours, Tours, France

University Hospital of Tours, Tours, France

University Hospital of Tours, Tours, France

University Hospital of Reims, Reims, France

University Hospital of Reims, Reims, France

Emmanuel Houdart - Lariboisière Hospital, Paris, France

Emmanuel Houdart - Lariboisière Hospital, Paris, France

University Hospital of Lille, Lille, France

University Hospital of Lille, Lille, France

University Hospital of Lille, Lille, France

University Hospital of Lille, Lille, France

University Hospital of Poitiers, Poitiers, France

University Hospital of Poitiers, Poitiers, France

Department of Neurology, Leuven University Hospital, and Vesalius Research Center, VIB, Leuven, Belgium

Department of Neurology, Erasme Hospital, Free University of Brussels and Laboratory of Experimental Neurology, ULB, Brussels, Belgium

Department of Neurology, Lille University Hospital - EA1046, France

Department of Neurology, Sainte- Anne University Hospital, Paris, France

Department of Neurology, Sainte- Anne University Hospital, Paris, France

Department of Neurology, Pitié-Salpêtrière University Hospital, Paris, France

Department of Neurology, Pitié-Salpêtrière University Hospital, Paris, France

Department of Neurology, Pitié-Salpêtrière University Hospital, Paris, France

Department of Neurology, Amiens University Hospital, France

Department of Neurology, Amiens University Hospital, France

Department of Neurology, Dijon University Hospital, France

Department of Neurology, Dijon University Hospital, France 
Department of Neurology, Besançon University Hospital, France

Department of Neurology, Besançon University Hospital, France

Department of Neurology, Klinikum Ludwigshafen, Germany

Department of Neurology, University Hospital of Munich, Germany

Department of Dermatology, Heidelberg University Hospital, Germany

Department of Rehabilitation Schmieder-Klinik, Heidelberg, Germany

Department of Rehabilitation Schmieder-Klinik, Heidelberg, Germany

Department of Neurology, Heidelberg University Hospital, Germany

Department of Neurology, Heidelberg University Hospital, Germany

Department of Clinical and Experimental Sciences, Neurology Clinic, Brescia University Hospital, Italy

Department of Clinical and Experimental Sciences, Neurology Clinic, Brescia University Hospital, Italy

Department of Clinical and Experimental Sciences, Neurology Clinic, Brescia University Hospital, Italy

Department of Clinical and Experimental Sciences, Neurology Clinic, Brescia University Hospital, Italy

Department of Clinical and Experimental Sciences, Neurology Clinic, Brescia University Hospital, Italy

Department of Clinical and Experimental Sciences, Neurology Clinic, Brescia University Hospital, Italy

Department of Clinical and Experimental Sciences, Clinica Medica, Brescia University Hospital, Italy

Department of Clinical and Experimental Sciences, Clinica Medica, Brescia University Hospital, Italy

Department of Clinical and Experimental Sciences, Clinica Medica, Brescia University Hospital, Italy

Department of Neurology, IRCCS Foundation Ca'Granda Hospital Policlinic Hospital, University of Milan, Italy

Department of Neurology, IRCCS Foundation Ca'Granda Hospital Policlinic Hospital, University of Milan, Italy

University of Milano Bicocca, San Gerardo Hospital, Monza, Italy

University of Milano Bicocca, San Gerardo Hospital, Monza, Italy

Milan Scientific Institute San Raffaele University Hospital, Italy

Department of Rehabilitation, Santa Lucia Hospital, Rome, Italy 
Department of Laboratory Medicine \& Advanced Biotechnologies, IRCCS San Raffaele Pisana, Rome, Italy

Department of Laboratory Medicine \& Advanced Biotechnologies, IRCCS San Raffaele Pisana, Rome, Italy

Stroke Unit and Division of Cardiovascular Medicine, University of Perugia Santa Maria della Misericordia Hospital, Sant'Andrea delle Fratte, Perugia, Italy

Department of Cerebrovascular Diseases, and Emilio Ciusani, PhD, Laboratory of Clinical Investigation, Fondazione IRCCS Istituto Neurologico Carlo Besta, Milano, Italy

Department of Cerebrovascular Diseases, and Emilio Ciusani, PhD, Laboratory of Clinical Investigation, Fondazione IRCCS Istituto Neurologico Carlo Besta, Milano, Italy

Department of Neurology, Basel University Hospital, Switzerland

Department of Neurology, Basel University Hospital, Switzerland

Department of Neurology, Basel University Hospital, Switzerland

Department of Neurology, Basel University Hospital, Switzerland

Stroke and Dementia Research Centre, St George's University of London, UK

Stroke and Dementia Research Centre, St George's University of London, UK

Institute of Neuroscience and Physiology, the Sahlgrenska Academy at University of Gothenburg, Gothenburg, Sweden

Institute of Neuroscience and Physiology, the Sahlgrenska Academy at University of Gothenburg, Gothenburg, Sweden

Department of Neurology, University Hospital of Istanbul, Turkey

Department of Neurology, University Hospital Sanatorio Allende, Cordoba, Argentina

Maryland Stroke Center, Department of Neurology, University of Maryland School of Medicine, Baltimore, Maryland, USA

Maryland Stroke Center, Department of Neurology, University of Maryland School of Medicine, Baltimore, Maryland, USA

Maryland Stroke Center, Department of Neurology, University of Maryland School of Medicine, Baltimore, Maryland, USA

Maryland Stroke Center, Department of Neurology, University of Maryland School of Medicine, Baltimore, Maryland, USA

Maryland Stroke Center, Department of Neurology, University of Maryland School of Medicine, Baltimore, Maryland, USA 
Department of Neurology, Erasmus MC University Medical Center, Rotterdam, the Netherlands

Department of Neurology, Erasmus MC University Medical Center, Rotterdam, the Netherlands

Department of Epidemiology Erasmus MC Rotterdam

Department of Epidemiology Erasmus MC Rotterdam

Department of Internal Medicine, Erasmus MC

Department of Internal Medicine, Erasmus MC

Laboratorio Neurovascular, Institut de Recerca, Hospital Vall d'Hebron, Barcelona, Spain

Laboratorio Neurovascular, Institut de Recerca, Hospital Vall d'Hebron, Barcelona, Spain

Imperial College Cerebrovascular Research Unit (ICCRU), Imperial College London, UK

Imperial College Cerebrovascular Research Unit (ICCRU), Imperial College London, UK

University of Utah, USA

Imperial College Cerebrovascular Research Unit (ICCRU), Imperial College London, UK

Imperial College Cerebrovascular Research Unit (ICCRU), Imperial College London, UK

Donders Institute for Brain, Cognition and Behaviour, Centre for Neuroscience, Department of Neurology, Radboud University Nijmegen Medical Centre, Nijmegen, The Netherlands

Departments of Neurology and Public Health Sciences University of Virginia, Charlottesville, Virginia, USA

Henry Ford Hospital, Detroit, Michigan, USA

Henry Ford Hospital, Detroit, Michigan, USA

Department of Neurology, Mayo Clinic, Jacksonville, Florida, USA

Department of Neurology, Mayo Clinic, Rochester, Minnesota, USA

Molecular Genetics Section, Laboratory of Neurogenetics, National Institute on Aging, NIH

Department of Molecular Neuroscience Institute of Neurology, University College London, London, United Kingdom

Department of Public Health Sciences and the Center for Public Health Genomics, University of Virginia, Charlottesville, Virginia, USA 
Department of Neurology, University Hospital of Giessen, Germany

Charité Universitätsmedizin Berlin, Department of Neurology, Berlin, Germany.

UCL Queen Square Institute of Neurology, London, UK

UCL Queen Square Institute of Neurology, London, UK

UCL Queen Square Institute of Neurology, London, UK

UCL Queen Square Institute of Neurology, London, UK

The James Cook University Hospital, Middlesbrough, UK

King's College Hospital NHS Foundation Trust, London, UK

University Hospital Southampton NHS Foundation Trust, Southampton UK

The National Hospital of Neurology and Neurosurgery, London, UK

UCL Queen Square Institute of Neurology, London, UK

The National Hospital of Neurology and Neurosurgery, London, UK

The National Hospital of Neurology and Neurosurgery, London, UK

Royal Preston Hospital, UK

St George's Hospital, London, UK

Hurstwood Park Neurological Centre, UK

Imperial Healthcare, Charing Cross Hospital, London, UK

Frenchay Hospital, Bristol, UK

Derriford Hospital, Plymouth, UK

Leeds General Infirmary, UK

Salford Royal Hospital, UK

The Walton Centre, Liverpool, UK

The Royal London Hospital, London, UK

Sheffield Teaching Hospitals NHS Trust, UK

Mayday Hospital, Croydon, UK

Mount Gould Hospital, Plymouth, UK

Newcastle University Hopsitals NHS Trust

Oxford Radcliffe Infirmary, UK

Oxford Radcliffe Infirmary, UK

Addenbrooke's Hospital, Cambridge, UK

Department of Neurology,Boston University School of Medicine, Framingham Heart Study, Boston, MA, USA 
University of Cologne,Germany

Aflac Cancer and Blood Disorder Center, Department of Pediatrics, Emory University School of Medicine, Atlanta, GA, USA

Filho Stroke and Cardiomyopathy Clinics,Federal University of Bahia, Brazilian National Research Committee (CNPq), Instituto Nacional de Ciencia e Tecnologiaem Doencas Tropicais (INCT-DT),Salvador,Brazil

Center for Human Genetic Research,Harvard Medical School,Boston, MA,USA Sunaina Yadav, Imperial College Cerebrovascular Research Unit, Department of Clinical Neuroscience,Imperial College London,London,UK

Medicine,Baltimore, MD,USA Department of Anesthesiology and Critical Care Medicine,Johns Hopkins

Department of Medical and Molecular Genetics, King's College London, SGDP Centre, Institute of Psychiatry, Psychology \& Neuroscience, King's College London,London,UK Bjorn Hansen, Department of Clinical Sciences Lund, Neurology,Lund University,Department of Neurology and Rehabilitation Medicine, Skåne University Hospital,Lund,Sweden

Sahlgrenska Academy at University of Gothenburg, Lund University,Department of Neurology and Rehabilitation Medicine,Skåne University Hospital,Lund,Sweden

Department of Clinical Sciences Lund, Cardiology,Lund University, The Section for Heart Failure and Valvular Disease, VO Heart and Lung medicine,Skane University Hospital,Lund,Sweden

Department of Neurology,Massachusetts General Hospital,Center for Human Genetic Research,Massachusetts General Hospital (MGH),Boston, MA,USA

Division of Behavioral Neurology, Department of Neurology,Massachusetts General Hospital,Center for Human Genetic Research,Massachusetts General Hospital (MGH),Boston, MA,USA

Department of Neurology,Massachusetts General Hospital,Center for Human Genetic Research,Massachusetts General Hospital (MGH),Boston, MA,USA

Center for Genomic Medicine,Massachusetts General Hospital (MGH),J. Philip Kistler Stroke Research Center, Department of Neurology, Massachusetts General Hospital (MGH),Boston, MA,USA

Department of Neurology,Massachusetts General Hospital, Harvard Medical School, Department of Neurology,J. Philip Kistler Stroke Research Center, MGH,Boston, MA,USA Ralph Sacco,Department of Neurology,Miller School of Medicine, University of Miami,Miami, FL,USA

Institute of Cardiovascular Research, Royal Holloway University of London,London,UK

Department of Neurology,Samsung Medical Center, Sungkyunkwan University School of Medicine, Seoul, Korea 
Department of Neurology,Samsung Medical Center, Sungkyunkwan University School of Medicine, Seoul, USA

Department of Neurology,Samsung Medical Center, Sungkyunkwan University School of Medicine, Seoul, Korea

Department of Pharmacology,School of Life Science and Biopharmaceutical Sciences, Shenyang Pharmaceutical University,Shenyang,China

Department of Internal Medicine,Taichung Veterans General Hospital, Taichung, Taiwan Steven Lubitz,Department of Cardiology,Massachusetts General Hospital, Harvard Medical School,Boston, MA,USA

Stroke Research Centre, Department of Brain Repair and Rehabilitation,University College London Institute of Neurology,London,UK

Reta Lila Weston Institute, UCL Institute of Neurology,University College London,London,UK

Reta Lila Weston Institute, UCL Institute of Neurology,University College London,London,UK

Department of Neuroradiology,University Hospital of Nantes,Nantes,France

MRC/BHF Cardiovascular Epidemiology Unit, Department of Public Health and Primary Care,University of Cambridge,Cambridge,UK

Department of Clinical Neurosciences, University of Cambridge,Cambridge,UK Anna Drazyk,Department of Clinical Neurosciences,University of Cambridge,Cambridge,UK

Stroke Research Group, Division of Clinical Neurosciences, University of Cambridge, Cambridge,UK

Neuroimaging Sciences, University of Edinburgh, Centre for Clinical Brain Sciences, University of Edinburgh, Edinburgh,UK

Department of Medicine,University of Maryland School of Medicine, Geriatrics Research and Education Clinical Center,Baltimore, Veterans Administration Medical Center,Baltimore, MD,USA

Department of Neurology,University of Maryland School of Medicine and Baltimore, VAMC,Baltimore, MD,USA

Centre for Healthy Brain Ageing, Psychiatry,University of New South Wales (UNSW),Sydney,Australia

Clinical Trial Service Unit and Epidemiological Studies Unit, Nuffield Department of Population Health,University of Oxford,Oxford,UK

Clinical Trial Service Unit and Epidemiological Studies Unit, Nuffield Department of Population Health,University of Oxford,Oxford,UK

Clinical Trial Service Unit and Epidemiological Studies Unit, Nuffield Department of Population Health,University of Oxford,Oxford,UK 
Departments of Neurology and Public Health Sciences, University of Virginia School of Medicine,Charlottesville, VA,USA

Cardiovascular Health Research Unit, Department of Medicine,University of Washington, Seattle, WA,USA

Comprehensive Stroke Care at the UW Medicine Stroke Center at Harborview Medical Center,University of Washington, Seattle, WA,USA

Department of Epidemiology,University of Washington, Fred Hutchinson Cancer Research Center, University of Washington, Seattle, WA,USA

Department of Neurology,Washington University School of Medicine,St. Louis, MO,USA Laura Heitsch,Department of Neurology, Radiology, and Biomedical Engineering,Washington University School of Medicine,St. Louis, MO,USA

Department of Neurology, Radiology, and Biomedical Engineering, Washington University School of Medicine,St. Louis, MO,USA

Department of Neurology,Aarhus University Hospital,Aarhus,Denmark

Department of Epidemiology and Population Health,Albert Einstein College of Medicine,New York, NY,USA

Department of Neurology and Stroke Unit;and Director of Clinical Epidemiology,,All India Institute of Medical Sciences (AIIMS),New Delhi,India

Department of Neurology,University of Massachusetts Medical School, Worchester, MA,USA

Neurology Clinic,Basel University Hospital,Basel,Switzerland

Beijing Hypertension League Institute,Beijing, China

Center of Stroke,Beijing Insitute for Brain Disorders,Department of Neurology, Beijing Tiantan Hospital, Capital Medical University,Beijing,China

Department of Neurology,Bordeaux University Hospital,Bordeaux,France

Department of Neurology,Bordeaux University Hospital,Bordeaux,France

Department of Biostatistics,Boston University,Boston,USA

Department of Neurology,University of California at Davis,Sacramento, CA,USA

Aleksandra Pikula,Department of Neurology,University of Toronto,Toronto,USA

Department of Neurology,Boston University School of Medicine,Boston,USA Philip Wolf,Boston University School of Medicine,Boston,USA

Boston University School of Public Health,Boston,USA

Boston University School of Public Health,Boston,USA

Rehabilitation department,Botucatu Medical School,Botucatu School of Medicine,University Estadual Paulista Júlio de Mesquita Filho, District of Rubião Junior,Botucatu,Brazil 
Division of Preventive Medicine,Brigham and Women's Hospital,Harvard Medical School,Boston, MA,USA

Department of Medicine,Brigham and Women's Hospital,Harvard Medical School,Boston,USA

Department of Neurology,Brigham and Women's Hospital, Massachusetts General Hospital,Boston, MA,USA

Center for Human Genetic Research, Massachusetts General Hospital (MGH),Division of Neurocritical Care and Emergency Neurology, Department of Neurology,Boston, MA,USA

Stroke Unit,Centro Hospitalar e Universitário de Coimbra, CHUC,Coimbra,Portugal Ales Tomek,Department of Neurology, 2nd Faculty of Medicine,Charles University in Prague and Motol University Hospital,Prague,Czech Republic

Department of Cardiology,Children's Hospital of Wisconsin,Milwaukee,USA Cara Carty,Children's Research Institute,Children's National Medical Center,Center for Translational Science, George Washington University, Washington,USA

Chinese Academy of Sciences (CAS),Beijing,China

Department of Neurological Sciences,Christian Medical College,Vellore,India Department of Neurological Sciences,Christian Medical College,Vellore,India Department of Neurological Sciences,Christian Medical College,Vellore,India Department of Pediatrics, College of Medicine, University of Oklahoma Health Sciences Center,Department of Pharmaceutical Sciences, College of Pharmacy, University of Oklahoma Health Sciences Center,Oklahoma City,USA

Department of Neurology,Columbia University,New York,USA

Neurological Institute of New York,Columbia University College,New York,USA Gudmar Thorleifsson,deCODE genetics/AMGEN inc,Reykjavik,Iceland

deCODE genetics/AMGEN inc,Reykjavik,Iceland University of Colorado Denver Anschutz Medical Campus,Denver, CO,USA Department of Neurology, Massachusetts General Hospital, Harvard Medical School, Boston, MA, USA,Department of Neurology, J. Philip Kistler Stroke Research Center, MGH,Boston, MA,USA

Translational Acute Brain Injury Research Center,Duke University,Durham,USA Department of Neurology,Duke University School of Medicine,Durham,USA Department of Medicine, Duke Stroke Center,Duke Univesrity School of Medicine,Durham, NC, USA

Cardiovascular and Stroke Genetics Working Group,Emory University,Atlanta,USA Carla Ibrahim-Verbaas,Department of Neurology,Erasmus MC, Rotterdam,Netherlands 
Department of Epidemiology,Erasmus MC, University Medical Center

Rotterdam,Clinic for Neurology and Psychiatry for Children and Youth,University of

Belgrade, Faculty of Medicine,Rotterdam,Netherlands

Department of Epidemiology,Erasmus University Medical

Center,Rotterdam,Netherlands Hieab Adams,Department of Epidemiology,Erasmus

University Medical Center,Rotterdam,Netherlands Fadi Charchar,L.E.W.

Carty Cardiovascular Genomics laboratory,Federation University

Australia,Ballarat,Australia

Stroke Division,Florey Institute of Neuroscience and Mental Health,Department of Neurology,Austin Health,Heidelberg,Australia

Laboratory of Cellular Neurobiology,Fondazione IRCCS Istituto Neurologico C. Besta, Cerebrovascular Disease Unit,Cerebrovascular Disease Unit,Fondazione IRCCS Istituto Neurologico C Besta,Milan,Italy

Department of Cerebrovascular Diseases,Fondazione IRCCS Istituto Neurologico Carlo Besta,Milan,Italy

Division of Public Health Sciences, Fred Hutchinson Cancer Research Center,Seattle,USA

Laboratory of Experimental Neurology,Free University of Brussels (ULB),Brussels,Belgium

Department of Neuroscience,Geisinger Health System,Danville,USA

Departement des neurosciences cliniques, Clinique de neurochirurgie,Geneva University Hospital,Geneva,Switzerland

Beth Israel Deaconess Medical Center,Harvard Medical School,Boston,USA caspar Grond-Ginsbach,Department of Neurology,Heidelberg University Hospital,Heidelberg,Germany

Department of Neurology,Helsinki University Central Hospital, Helsinki,Finland

Department of Neurology,Helsinki University Central Hospital, Clinical Neurosciences, Neurology,University of Helsinki,Helsinki,Finland

Department of Neurology,Helsinki University Central Hospital, Helsinki,Finland Hanne Sallinen,Department of Neurology,Helsinki University Hospital, Helsinki,Finland

Department of Neurology,Heritage Hospital,Department of Neurology,Neuro Expert Clinic,Varanasi,India

Department of Neurology,Hiedelberg University Hospital,Heidelberg,Germany Pablo Bonardo,Department of Neurology,Hospital Británico

Ciudad Autónoma de Buenos Aires,Argentina

Hospital Geral de Fortaleza/SUS Fortaleza,Ceará,Brazil

Hospital Governador Celso Ramos,Florianópolis,Brazil 
Stroke Pharmacogenomics and Genetics,Hospital Mútua de Terrassa, Fundació Docència i Recerca Mútua Terrassa,Terrassa,Spain

Department of Neurology,Hospital Rafael A. Calderon Guardia,San Jose,Costa Rica Melanie Laarman,Cardiac Development and Genetics Group,Hubrecht Institute,Utrecht,Netherlands Roberta Carriero,Inflammation and immunology laboratory,Humanitas Clinical Institute,Pavia,Italy Elizabeth Holliday,Public Health Stream,Hunter Medical Research Institute,Faculty of Health and Medicine,University of Newcastle,New Lambton,Australia

Neurology,Hygeia Hospital,Marousi,Greece

Department of Genetic and Genomic Sciences, Icahn School of Medicine,New York,USA

Department of Genetic and Genomic Sciences, Icahn School of Medicine, Mount Sinai,New York,USA

Icelandic Heart Association Research Institute,Faculty of Medicine,University of Iceland,Kopavogur,Iceland

Neurovascular Research Group (NEUVAS). Neurology Department., IMIM Hospital del Mar,Barcelona,Spain

Department of Neurology, Neurovascular Research Group,IMIM-Hospital del Mar,Barcelona,Spain

Neurology Service,IMIM-Hospital del Mar, Institut Hospital del Mar d'investigacions Mèdiques,Barcelona,Spain

Faculty of Medicine Department of Medicine, Imperial College London,London,UK Dipender Gill,Centre for Pharmacology and Therapeutics, Imperial College London, London,UK Ganesh Chauhan, Centre for Brain Research,Indian Institute of Science,Bangalore,India

UMR-S 740,INSERM,Paris,France Sara Kaffashian,INSERM U1219 Bordeaux Population Health Research Center,Bordeaux,France Cecilia Samieri,INSERM U1219 Bordeaux Population Health Research Center,ISPED,University of Bordeaux,Bordeaux,France

INSERM U1219 Bordeaux Population Health Research Center,Department of Public Health,CHU Bordeaux,Bordeaux,France

INSERM U1219 Bordeaux Population Health Research Center, Bordeaux, France,University of Bordeaux,France

INSERM U1219 Bordeaux Population Health Research Center, Bordeaux, France,University of Bordeaux,France

Data Analytics Department, Institute for Infocomm Research (I2R),Singapore,Singapore

Institute of Biomedical And Genetic Engineering,Islamabad,Pakistan 
Institute of Biomedical Sciences, Academia Sinica, Taipei,Taiwan

Institute of Biomedical Sciences, Academia Sinica, Graduate Institute of Integrated Medicine, China Medical University, Taipei,Taiwan

Computational Biology Unit,Institute of Molecular Genetics-National Research Council,Pavia,Italy

Department of Clinical Neuroscience, Institute of Neuroscience and Physiology, Sahlgrenska Academy at University of Gothenburg, Gothenburg,Sweden

Department of Clinical Neuroscience, Institute of Neuroscience and Physiology, Sahlgrenska Academy at University of Gothenburg, Sahlgrenska University Hospital,Gothenburg,Sweden

Department of Clinical Neuroscience,Institute of Neuroscience and Physiology, Sahlgrenska Academy at University of Gothenburg, Gothenburg,Sweden

Stroke Clinic,Instituto Nacional de Neurologia y Neurocirugia, Manuel Velasco Suarez,Mexico City,Mexico

Department of Molecular Neuropharmacology,Polish Academy of Sciences, Warsaw,Poland

Laboratory of Epidemiology and Population Science, Intramural Research Program, National Institutes of Health, National Institute on Aging,Bethesda,USA

Ion Torrent by Life Technologies (company),Carnegie Mellon University,San Francisco,USA

Cerebrovascular Unit,IRCCS Foundation C. Besta Neurological Institute,Milan, Italy Tomasz Dziedzic,Department of Neurology,Jagiellonian University,Krakow,Poland Jagiellonian University,Krakow,Poland Jagiellonian Centre for Experimental Therapeutics,Jagiellonian University, Krakow,Poland

Department of Neurology,Jagiellonian University Medical College,Krakow,Poland Agnieszka Slowik,Department of Neurology,Jagiellonian University Medical College,Krakow,Poland Andrzej Urbanik, Department of Radiology,Jagiellonian University Medical College,Krakow,Poland Jerzy Gasowski,Department of Internal Medicine and Gerontology,Jagiellonian University Medical College,Krakow,Poland Rafa? Olszanecki,Faculty of Medicine,Jagiellonian University Medical College,Krakow,Poland Jagiellonian University Medical College,Poland John Hunter Hospital, Hunter Medical Research Institute and University of Newcastle,Newcastle,Australia

Department of Neurology,Johns Hopkins Medicine,University of Pittsburgh School of Medicine,Baltimore,USA 
Department of Clinical Neuroscience, Karolinska Institute,Stockholm,Sweden Milita Crisby,Division of Clinical Geriatrics, Neurobiology Care Sciences and Society,Karolinska Institute,Huddinge,Sweden Lina Keller,Karolinska Institutet,Stockholm,Sweden Kristina Schlicht,Institut für Medizinische Informatik und Statistik,Kiel University,Kiel,Germany

Social genetic and Developmental Psychiatry,King's College London,London, Christof Haffner,Institute for Stroke and Dementia Research,Klinikum der Universität München,Munich,Germany

Institute for Stroke and Dementia Research,Klinikum der Universität München, Ludwig-Maximilians-University LMU,Munich,Germany

Krakow University Hospital,Krakow,Poland

Department of Neurosciences, Experimental Neurology,KU Leuven - University of Leuven,Department of Neurology,University Hospitals Leuven, VIB Center for Brain \& Disease Research, Leuven,Belgium

Department of Epidemiology and Public Health,Kyushu University, Graduate School of Medical Sciences,Fukuoka,Japan

Department of Epidemiology and Public Health,Kyushu University, Graduate School of Medical Sciences, Fukuoka,Japan

School of Psychological Science,La Trobe University,Melbourne,Australia

Department of Neurology,Landspítali Háskólasjúkrahús, Reykjavík,Iceland Saskia Lesnik Oberstein,Department of Clinical Genetics,Leiden University Medical Center,Leiden,Netherlands

Neurology department and Stroke center, Linkou Chang Gung Memorial Hospital,Taipei,Taiwan

Lomonosov Moscow State University,Moscow,Russia

Institute for Stroke and Dementia Research,Ludwig-Maximilians-University, Klinikum der Universität München,Munich,Germany

Institute for Stroke and Dementia Research,Ludwig-Maximilians-University, Klinikum der Universität München, Munich Cluster for Systems Neurology (SyNergy),Munich,Germany

Department of Clinical Sciences Lund, Neurology,Lund University,Department of Neurology and Rehabilitation Medicine,Skåne University Hospital,Lund,Sweden

Department of Clinical Sciences Malmö,Lund University,Vascular Center,Skåne University Hospital,Malmö,Sweden

Diagnostic Radiology,Lund University,Lund,Sweden Olle Melander,Department of Clinical Sciences, Lund University,Lund,sweden

Geriatric Medicine,Lund University,Lund,Sweden

Neurology,Lund University,Lund,Sweden 
Department of Neurology,Maastricht University Medical Center (MUMC+), Cardiovascular Research Institute Maastricht (CARIM),Maastricht,Netherlands

Medical Genetics Research Center (MGRC),Mashhad University of Medical Sciences (MUMS), School of Medicine,Department of Medical Genetics,Mashhad University of Medical Sciences (MUMS), Faculty of Medicine,Mashhad,Iran

Center for Genomic Medicine, Massachusetts General Hospital,Boston, MA,USA Jose Florez,Center for Human Genetic Research, MGH,Massachusetts General Hospital, Harvard Medical School, Boston, MA,USA

Massachusetts General Hospital,South Hadley, MA,USA

Department of Neurology,Massachusetts General Hospital,Boston, MA,USA

Department of Neurology,Massachusetts General Hospital,Boston, MA,USA

Department of Neurology, JPK Stroke Research Center,Massachusetts General Hospital and Harvard Medical School, Department of Radiology, Athinoula A Martinos Center for Biomedical Imaging, MGH,Boston, MA,USA

Department of Neurology, J. Philip Kistler Stroke Research Centre,Massachusetts General Hospital and Harvard Medical School,Boston,USA

Division of Neurocritical Care and Emergency Neurology, Department of Neurology,Massachusetts General Hospital, Harvard Medical School,J. P. Kistler Stroke Research Center,Massachusetts General Hospital, Harvard Medical School,Boston, MA,USA

Neurology, Anatomy \& Neurobiology, and Physical Medicine \& Rehabilitation,University of California Irvine,Irvine,USA

The Computer Science and Artificial Intelligence Laboratory, Massachusetts Institute of Technology,Cambridge,USA

Myhsok Max-Planck-Institute of Psychiatry,Munich,Germany

Department of Neurology,Mayo Clinic,Rochester, MN,USA

Department of Neurology,Mayo Clinic,Jacksonville,USA

Department of Medical Genetics and Neuroscience,Mayo Clinic,Jacksonville,USA Thomas Brott,Neurology,Mayo Clinic, Department of Surgery, University of Medicine and Dentistry of New Jersey,Jacksonville, FL,USA

Population Health Research Institute,McMaster University,Hamilton,Canada

Population Health Research Institute,McMaster University,Hamilton,Canada

Department of Neurology,Medical school of Alexandria,Alexandria Governorate,Egypt

Department of Adult Neurology,Medical University of Gdansk \& University Clinical Centre in Gdansk,Gdansk,Poland 
Department of Neurology,Medical University of Graz,Graz,Austria Helena Schmidt, Institute of Molecular Biology and Biochemistry,Medical University of Graz,Graz,Austria

Department of Neurology,Medical University of Graz,Graz,Austria

Department of Neurology,Medical University of Graz,Graz,Austria

Department of Neurology,Medical University of Graz,Graz,Germany

Department of Neurology,Medical University of South Carolina,Charleston,USA

Department of Human Genomics,Mie University, Life Scinece Research

Center,Mie,Japan

Department of Neurology,Miller School of Medicine, University of Miami,Miami, FL,USA

Hussman Institute for Human Genomics,Miller School of Medicine, University of Miami,Miami, FL,USA

Hussman Institute for Human Genomics,Miller School of Medicine, University of Miami,Miami, FL,USA

Department of Neurology, College of Medicine,National Cheng Kung University, Tainan, Taiwan

Cardiovascular Epidemiology and Human Genomics Branch,National Heart, Lung and Blood Institute, Intramural Research,Veterans Administration Healthcare,Center for Population Genomics,Boston, MA,USA

National Institute of Neurological Disorders and Stroke,National Institute of Health, University of California,Bethesda, MD,USA

National Institute of Pharmaceutical Education and Research,Ahmedabad,India Andrew Singleton,Laboratory of Neurogenetics,National Institute on Aging, Intramural Research Program,Bethesda, MD,USA

National Institute of Neurological Disorders and Stroke,National Institutes of Health,Bethesda, MD,USA

General neurology and stroke,National University Hospital,Singapore,Singapore Department of Pharmacology,National University of Singapore,Singapore,Singapore Institute of Neuroscience,Newcastle University,Newcastle, UK

Division of Neuroscience, Stroke Program in the Neural Environment, NINDS,Bethesda, MA,USA

Department of Environmental Studies,North Eastern Hill University (NEHU),Shillong, India

Obafemi Awolowo University,lle-Ife,Nigeria Bishwa Sapkota,Harold Hamm Diabetes Center,Oklahoma University,Oklahoma City,USA Rebecca Jackson,Center for women's health, Ohio State University Wexner Medical Center,Colombus,USA 
Parc de Salut Mar,Barcelona,Spain

Department of Genetics,Perelman School of Medicine, University of Pennsylvania,Pennsylvania, PA,USA

Department of Neurology,Radboud University Medical

Center,Nijmegen,Netherlands

Department of Neurology,Radboud University Medical Centre, Donders Institute for Brain, Cognition and Behaviour,Department of Neurology and Neurosurgery,University Medical Center, Brain Center Rudolf Magnus,Nijmegen,Netherlands

Department of Clinical Medicine,Rigshospitalet - Neurocentret,Glostrup,Denmark Laboratory for Statistical Analysis,RIKEN Center for Integrative Medical Sciences,Center for Genomic Medicine, Kyoto University Graduate School of Medicine,Yokohama,Japan

RIKEN Center for Integrative Medical Sciences, Yokohama,Japan Yukinori Okada,Laboratory for Statistical Analysis,RIKEN Center for Integrative Medical Sciences,Department of Statistical Genetics, Osaka University Graduate School of Medicine, Yokohama,Japan

Stroke Prevention \& Atherosclerosis Research Centre,Robarts Research,London (ON), Canada

Västra Vötalandsregionen,Sahlgrenska Academy at University of Gothenburg,Gothenburg,Sweden

Department of Medical and Clinical Genetics, Institute of Biomedicine,Sahlgrenska Academy at University of Gothenburg, Gothenburg,Sweden

Department of Clinical Neurosciences/Neurology,Sahlgrenska Academy at University of Gothenburg, Institute of Neuroscience and Physiology,Sahlgrenska University Hospital,Gothenburg,Sweden

Neurology Department,San Raffaele Scientific Institute,University Vita-Salute San Raffaele,Milan,Italy

Department of Neurology,Sanatorio Allende,Cordoba,Argentina Rodrigo Bazan,Department of Neurology,São Paulo State University (Unesp) Botucatu Medical School,Botucatu,Brazil

Hospital das Clínicas,São Paulo State University (Unesp) Botucatu Medical School,Botucatu,Brazil

School of Life Science, University of Lincoln,Lincoln,UK

Medicine department,School of Medicine, University of Maryland,Baltimore,USA

Cardiovascular Medicine,School of Medicine, University of Stanford,Stanford,USA Jamie Wright, Anderson Cancer Center, MD Anderson Clinical Cancer Genetics,School of Medicine, University of Texas,Houston,USA 
Scientific Center of Neurology,Moscow,Russia

National Neuroscience Institute,Singapore General Hospital,Department of Pediatrics,National University of Singapore, Yong Loo Lin School of Medicine,Singapore,Singapore Eng King Tan,Department of Neurology,Singapore General Hospital, National Neuroscience Institute,Neuroscience and Behavioral Disorders Program,Duke-National University of Singapore Graduate Medical School,Singapore,Singapore

Department of Cardiology,Skane University Hospital,Malmö,Sweden

Critical Care Neurology,Skåne University Hospital, Lund university,Malmö,Sweden Christian Opherk,Department of Neurology,SLK-Kliniken

Heilbronn,Germany Chaeyoung Lee,Department of Bioinformatics and Life Science,Soongsil University,Seoul,Korea David Tregouet,Team Genomics and Pathophysiology of Cardiovascular Diseases,Sorbonne Universités, UPMC Univ Paris 06, INSERM, UMR_S 1166,ICAN Institute for Cardiometabolism and Nutrition,Paris,France

Department of Neurology,Southern Medical University, FIMMU,Guangzhou,China Hung Yi Chiou,School of Public Health, College of Public Health and Nutrition,Taipei Medical University,Taipei,Taiwan

Department of Health Information Management,Tehran University of Medical Sciences (TUMS), Tehr?n,Iran

Garrison Institute on Aging,Texas Tech University Health Sciences Center,Lubbock,USA

Division of Neurology, Department of Medicine and Therapeutics, The Chinese University of Hong Kong,Hong-Kong,Hong-Kong

Division of Neurology, Department of Medicine and Therapeutics, The Chinese University of Hong Kong,Hong-Kong,Hong-Kong

Department of Medicine and Therapeutics, The Chinese University of Hong Kong,Hong- Kong,Hong-Kong

Department of Obstetrics and Gynecology, The Chinese University of Hong Kong,Hong-Kong,Hong-Kong

Department of Pathology and Genetics, The Sahlgrenska Academy at University of Gothenburg, Institute of Biomedicine,Gothenburg,Sweden

Department of Neurology and Neurosurgery,The Urals State Medical University, International Headache Center 'Europe-Asia',Yekaterinburg,Russia

Department of Cardiovascular Medicine, The Wellcome Trust Centre for Human Genetics,Oxford,UK

Department of radiology,Tianjin Medical University (TIJMU), Tianjin,China

Tunku Abdul Rahman University, UTAR,Kampar Perak,Malaysia 
Stroke Research Center,UCL Institue of Neurology,London,UK

Department of Neurology and Neurosurgery,UMC Utrecht, Brain Center

Utrecht,Netherlands

Universidade da Região de Joinville,Joinville,Brazil

Department of Medicine,Universidade da Região de Joinville -

Univille,Joinville,Brazil Norberto Cabral, Department of Medicine,Universidade da

Região de Joinville (Univille),Joinville,Brazil

Molecular Genetics Laboratory,Universidade Estadual de Campinas, Faculdade de Ciências Médicas,Campinas,Brazil

Neurovascular Research Laboratory, Vall d'Hebron Institut of Research, Neurology and Medicine Departments,Universitat Autònoma de Barcelona, Vall d'Hebrón Hospital,Stroke Pharmacogenomics and Genetics,Fundacio Docència i Recerca Mutua Terrassa,Spain

Neurovascular Research Laboratory, Vall d'Hebron Institut of Research, Neurology and Medicine Departments,Universitat Autònoma de Barcelona, Vall d'Hebrón Hospital,Stroke Pharmacogenomics and Genetics,Fundacio Docència i Recerca Mutua Terrassa,Barcelona,Spain

Neurovascular Research Laboratory,Universitat Autònoma de Barcelona, Vall d'Hebron Institute of Research,Neurology Department,Hospital Vall d'Hebron,Barcelona,Spain

Institute for Human Genetics, The Center for Cerebrovascular Research (CCR),Université de Californie,San Francisco,USA

Albrecht Kossel Institute,University Clinic of Rostock,Rostock,Germany

Department of Neurology,University College Hospital,Ibadan,Nigeria Reecha Sofat,Metabolism \& Experi Therapeutics, Div of Medicine, Faculty of Medical Sciences, University College London, London,UK

University Medical Center Utrecht,Utrecht,Netherlands Dominique PV de Kleijn,Laboratory of Experimental Cardiology,University Medical Center Utrecht,ICIN-Netherlands Heart Institute,Utrecht,Netherlands

Department of Neurology and Neurosurgery,University Medical Center Utrecht, Brain Center Rudolf Magnus, The Dutch Parelsnoer Institute-Cerebrovascular accident (CVA) Study Group,Utrecht,Netherlands

Department of Neurology and Neurosurgery,University Medical Center Utrecht, Brain Center Rudolf Magnus, Utrecht,Netherlands

Department of Neurology,University Medical Center Utrecht, Brain Center

Utrecht,Netherlands 
Department of Neurology and Neurosurgery,University Medical Center Utrecht, Brain Center Rudolf Magnus, Julius Center for Health Sciences and Primary Care,University Medical Center Utrecht,Utrecht,Netherlands

Laboratory of Experimental Cardiology,University Medical Center Utrecht, Division of Heart and Lungs, Utrecht,Netherlands

Dept of Medicine \& Therapeutics, University of Aberdeen, King's

College,Aberdeen,UK

School of Public Health,University of Alabama,Birmingham, AL,USA

Department of Biostatistics, University of Alabama,Birmingham, AL,USA

Department of Epidemiology,University of Alabama at Birmingham,Birmingham, AL,USA

Neurology,University Of Alabama Hospital, Spectrum Health Butterworth Campus,Birmingham, AL,USA

Department of Epidemiology,University of Alabama School of Medicine,Birmingham, AL,USA

Department of Neurology,University of Arizona,Stroke Center at Georgetown,University of Georgetown,Tucson, AZ,USA

Neurology Clinic, Clinical Center of Serbia,University of Belgrade, Faculty of Medicine,Belgrade, Serbia

University of Bordeaux,INSERM U1219,Bordeaux,France

INSERM U1219 Bordeaux Population Health Research Center, University of Bordeaux,Bordeaux,France

University of Bordeaux,Bordeaux, Alessandro Padovani,Department of Clinical and Experimental Sciences,University of Brescia,Brescia,Italy

Department of Clinical and Experimental Sciences,University of Brescia, Neurology Clinic,Brescia,Italy

Department of Neurology,University of Cairo,Cairo,Egypt

Alzheimer's Disease Center,University of Californa Davis,Sacramento, CA, USA Yuqi Zhao,Department of Integrative Biology and Physiology,University of California, Los Angeles, CA,USA

Department of Neurology,University of California, Los Angeles, CA,USA

Stroke Research Group, Division of Clinical Neurosciences, University of Cambridge,Cambridge,UK

Department of Clinical Neurosciences,University of Cambridge,Cambridge,England MRC/BHF Cardiovascular Epidemiology Unit, Department of Public Health and Primary Care,, University of Cambridge, The National Institute for Health Research 
Blood and Transplant Research Unit in Donor Health and Genomics, Wellcome Trust Sanger Institute,Cambridge,UK

Department of Clinical Neurosciences, Neurology Unit,University of Cambridge,Cambridge,UK

Jacobs,Stroke Research Group,University of Cambridge, Division of Clinical Neurosciences,DZNE,Cambdrige,UK

School of Medical Sciences, FCM,University of Campinas, UNICAMP,São Paulo,Brazil

Department of Neurology,University of Campinas, UNICAMP,São Paulo,Brazil

Gardner Neuroscience Institute, Comprehensive Stroke Center,University of Cincinnati,Cincinnati, $\mathrm{OH}, \mathrm{USA}$

University of Cincinnati College of Medicine,Cincinnati, $\mathrm{OH}, \mathrm{USA}$

Department of Neurology and Rehabilitation Medicine,University of Cincinnati Gardner Neuroscience Institute, Comprehensive Stroke Center,Cincinnati, OH,USA Institute for Cancer Genetics,University of Columbia,New York,USA

Centre for Clinical Brain Sciences,University of Edinburgh, Edinburgh,UK

Centre for Medical Informatics, Usher Institute, University of Edinburgh, Edinburgh,UK

Centre for Medical Informatics, Usher Institute,University of

Edinburgh,Edinburgh,UK

College of Pharmacy,University of Florida,Gainesville, FL,USA

Department of Neurology,University of Florida College of Medicine,Jacksonville, FL,USA

Department of Pharmacotherapy and Translational Research and Center for Pharmacogenomics, University of Florida, College of Pharmacy,Gainesville, FL,Japan USA

Insittute of Cardiovascular and Medical Sciences,University of Glasgow,Glasgow,UK

School of Medicine, Dentistry and Nursing,University of Glasgow,Glasgow,UK Martin Söderholm,Bioinformatics Core Facility,University of Gothenburg,Gothenburg,Sweden

Bioinformatics Core Facility,University of Gothenburg,Gothenburg,Sweden

University of Gothenburg,Gothenburg,Sweden

University of Gothenburg,Gothenburg,Sweden

University of Gothenburg,Gothenburg,Sweden

University of Hawaii, John A. Burns School of Medicine,Department of Neurology,The Queen's Medical Center,Honolulu,USA 
Institute for Advanced Medical Research and Training,University of Ibadan, College of Medicine, Ibadan,Nigeria

Institute of Cardiovascular Research Royal Holloway,University of London, London,UK

Medicine department,University of Maryland,Animal Genetics Improvement Laboratory,United States Department of Agriculture,Baltimore, MD,USA

Department of Neurology,University of Maryland,Baltimore, MD,USA

Department of Neural and Pain Sciences, University of Maryland,Baltimore, MD,USA Patrick McArdle,Division of Endocrinology, Diabetes and Nutrition,University of Maryland School of Medicine,Baltimore, MD,USA

Bioinformatics,University of Maryland School of Medicine,Baltimore, MD,USA

Epidemiology \& Public Health,University of Maryland School of Medicine,Baltimore, MD,USA

Department of Neurology,University of Maryland School of Medicine and Baltimore, VAMC, MD, USA,Baltimore, MD,USA

University of Melbourne,Melbourne,Australia

Department of Neurovascular,University of Michigan Medical School,Ann Arbor,USA Mario Di Napoli,Department of Neurology,University of Naples,Naples, Italy

Comprehensive Cancer Center,University of New Mexico, Albuquerque, NM,USA

Neurology,University of New Mexico,Albuquerque, NM, USA

School of Medicine and Public Health,University of Newcastle,New Lambton,Australia Shahbaz Zamani,University of Newcastle,Newcastle,Australia

Cell Biology and Physiology,University of North Carolina,Chapel Hill,USA

Nuffield Department of Clinical Neurosciences,University of Oxford,Oxford,UK

Nuttfield Department of Population Health,University of Oxford,Oxford,UK

CTSU-Clinical Trial Service Unit and Epidemiological Studies Unit,University of Oxford,Oxford,UK

Department of Neurology,University of Pisa,Pisa, Italy

Department of Neurology,University of Sao Paulo,Ribeirao Preto,Brazil

Genetic, Diagnostic and Research Laboratory, Department of Anatomy,University of Sri Jayewardenepura, Faculty of Medical Sciences,Nugegoda,Sri Lanka

Department of Neurology and Neurosurgery,University of Tartu,Tartu,Estonia Department of Neurology and Neurosurgery,University of Tartu,Tartu,Estonia Faculty of Health,University of Technology Sydney,Ultimo,Australia 
Brown Foundation Institute of Molecular Medicine // Human Genetics

Center,University of Texas Health Science Center at Houston,Houston, TX,USA

University of Texas Medical School,Houston, TX,USA

Division of Medical Genetics,University of TexasHealth Science Center, McGovern Medical School,Department of Internal Medicine,University of TexasHealth Science Center, McGovern Medical School,Houston, TX,USA

Neurology,University of Utah,Salt Lake City, UT,USA

Department of Neurology,University of Utah,Salt Lake City, UT,USA

Neurobiology and Anatomy, University of Utah School of Medicine,Salt Lake City, UT,USA

Radiology and Imaging Sciences, University of Utah School of Medicine,Neurosurgery, University of Utah School of Medicine,Salt Lake City, UT,USA

Department of Hematology and oncology,University of Vermont, Medical Center,Colchester,USA

Center for Public Health Genomics,University of Virginia,Charlottesville, VA,USA Andrew Southerland, Departments of Neurology and Public Health Sciences,University of Virginia,Charlottesville, VA,USA

Newborn Intensive Care Units,University of Virginia Children's Hospital,Charlottesville, VA,USA

Center for Public Health Genomics, University of Virginia School of Medicine,Charlottesville, VA,USA

Center for Public Health Genomics, University of Virginia School of Medicine,Charlottesville, VA,USA

Cardiovascular Health Research Unit, Department of Medicine,University of Washington,Department of Epidemiology,University of Washington,Seattle, WA,USA

Department of Epidemiology,University of Washington,Department of Neurology,University of Washington,Seattle,USA

Department of Neurology,University Teaching Hospital,Lusaka,Zambia

Wake Forest Baptist Health,Winston-Salem, NC, USA

Center for Public Health Genomics and Department of Biostatistical Sciences, Wake Forest School of Medicine,Winston-Salem, NC,USA

Department of Neurology,Wake Forest University Medical Center,Winston-Salem, NC,USA

Department of Psychiatry, The Hope Center Program on Protein Aggregation and Neurodegeneration (HPAN), Washington University School of Medicine,Department 
of Developmental Biology, Washington University School of Medicine,St. Louis, MO,USA

Department of Psychiatry, The Hope Center Program on Protein Aggregation and Neurodegeneration (HPAN), Washington University, School of Medicine,St. Louis, MO,USA

West China Medical School, Sichuan University,Sichuan,China

Department of Neurology, Neuro Intensive Care \& Neurological Emergencies, Yale School of Medicine,Nw Haven, CT,USA

Department of Neurology, Yale University School of Medicine, Program in Medical and Population Genetics, The Broad Institute of Harvard and MIT,New Haven, CT, USA

Philip Kistler Stroke Research Center,Massachusetts General Hospital,USA

${ }^{1}$ Department of Neurology and Neurosurgery, University Medical Center Utrecht Brain Center, Utrecht University, Utrecht, The Netherlands

${ }^{2}$ Department of Pathology and Immunology, Faculty of Medicine, University of Geneva, Geneva, Switzerland

${ }^{3}$ Neurosurgery Division, Department of Clinical Neurosciences, Faculty of Medicine, Geneva University Hospitals, Geneva, Switzerland

${ }^{4}$ Université de Nantes, CHU Nantes, INSERM, CNRS, l'institut du thorax, Nantes, France

${ }^{5} \mathrm{CHU}$ Nantes, Department of Neuroradiology, Nantes, France

${ }^{6}$ Stroke Research Centre, University College London Queen Square Institute of Neurology, London, UK

${ }^{7}$ Department of Neurosurgery, Klinikum rechts der Isar, Technical University Munich, Munich, Germany

${ }^{8}$ Stroke Research Centre, University College London, Institute of Neurology, London, UK

${ }^{9}$ Laboratory for Statistical and Translational Genetics, RIKEN Center for Integrative Medical Sciences, Yokohama, Japan

${ }^{10}$ Department of Cancer Biology, Institute of Medical Science, The University of Tokyo, Tokyo, Japan

${ }^{11}$ Department of Ophthalmology, Graduate School of Medical Sciences, Kyushu University, Fukuoka, Japan

${ }^{12}$ Department of Ocular Pathology and Imaging Science, Graduate School of Medical Sciences, Kyushu University, Fukuoka, Japan

${ }^{13}$ Graduate School of Frontier Sciences, The University of Tokyo, Tokyo, Japan 
${ }^{14}$ Clinical Trial Service Unit and Epidemiological Studies Unit, Nuffield Department of Population Health, University of Oxford, Oxford, U.K

${ }^{15}$ Medical Research Council Population Health Research Unit, University of Oxford, Oxford, U.K

${ }^{16}$ School of Public Health, Peking University Health Science Center, Beijing, China

${ }^{17}$ Montreal Neurological Institute and Hospital, McGill University, Montréal, QC, Canada

${ }^{18}$ Lady Davis Institute, Jewish General Hospital, McGill University, Montréal, QC, Canada

${ }^{19}$ Centre for Medical Informatics, Usher Institute, University of Edinburgh, Edinburgh, UK

20UK Biobank, Cheadle, Stockport, UK

${ }^{21}$ Neurogenetics Laboratory, The National Hospital of Neurology and Neurosurgery, London, UK

${ }^{22}$ Pediatric Radiology, Necker Hospital for Sick Children, Université Paris Descartes, Paris, France

${ }^{23}$ Department of Neuroradiology, Sainte-Anne Hospital and Université Paris Descartes, INSERM UMR S894, Paris, France

${ }^{24}$ Department of Neuroradiology, University hospital of Brest, Brest, France

${ }^{25}$ Department of Neuroradiology, Pitié-Salpêtrière Hospital, Paris, France

${ }^{26}$ Department of Neuroradiology, University hospital of Rennes, Rennes, France

${ }^{27}$ Department of Research, Innovation and Education, Division of Clinical Neuroscience, Oslo University Hospital, Oslo, Norway

${ }^{28}$ K. G. Jebsen Center for Genetic Epidemiology, Department of Public Health and Nursing, Faculty of Medicine and Health Sciences, Norwegian University of Science and Technology, Trondheim, Norway

${ }^{29}$ Research and Communication Unit for Musculoskeletal Health (FORMI), Department of Research, Innovation and Education, Division of Clinical Neuroscience, Oslo University Hospital, Oslo, Norway

${ }^{30}$ Institute of Clinical Medicine, Faculty of Medicine, University of Oslo, Oslo, Norway

${ }^{31}$ Department of Public Health and Nursing, Faculty of Medicine and Health Sciences, Norwegian University of Science and Technology, Trondheim, Norway

${ }^{32}$ The Cancer Clinic, St Olavs Hospital, Trondheim University Hospital, Trondheim, Norway

${ }^{33}$ Department of Internal Medicine, Division of Cardiovascular Medicine, University of Michigan, Ann Arbor, 48109, MI, USA 
${ }^{34}$ HUNT Research Center, Department of Public Health and Nursing, Faculty of Medicine and Health Sciences, Norwegian University of Science and Technology, Trondheim, Norway

35Julius Center for Health Sciences and Primary Care, University Medical Center Utrecht, Utrecht, the Netherlands

${ }^{36}$ National Institute for Public Health and the Environment, Bilthoven, the Netherlands

${ }^{37}$ University of Applied Science and Arts, Dortmund

${ }^{38}$ Institute for Medical Informatics, Biometry and Epidemiology (IMIBE), University Hospital Essen, 45122 Essen, Germany

${ }^{39}$ Zurich University of Applied Sciences, School of Life Sciences and Facility Management

${ }^{40}$ SIB Swiss Institute of Bioinformatics

${ }^{41} \mathrm{~A}$ list of members and affiliations appears in the Supplementary Note

${ }^{42}$ Department of Surgery, University of Otago, Dunedin, New Zealand

${ }^{43}$ Department of Cardiovascular Sciences and National Institute for Health Research, University of Leicester, UK

${ }^{44}$ Leicester Biomedical Research Centre University of Leicester Glenfield Hospital Leicester United Kingdom

${ }^{45}$ Department of Neurology, University of California at San Francisco, San Francisco, USA

${ }^{46}$ Department of Anesthesia and Perioperative Care, Center for Cerebrovascular Research, University of California, San Francisco, California, USA

${ }^{47}$ Department of Epidemiology and Biostatistics, University of California, San Francisco, California, USA

${ }^{48}$ Institute for Human Genetics, University of California, San Francisco, California, USA

${ }^{49}$ Social, Genetic and Developmental Psychiatry Centre, Institute of Psychiatry, Psychology and Neuroscience, King's College London, London, SE5 8AF, UK 50UK National Institute for Health Research (NIHR) Biomedical Research Centre (BRC), South London and Maudsley NHS Foundation Trust, London, SE5 8AF, UK ${ }^{51}$ Division of Research, Kaiser Permanente of Northern California, Oakland, California, USA

${ }^{52}$ Department of Neurology, Donders Institute for Brain, Cognition and Behaviour, Radboud University Medical Center, Nijmegen, The Netherlands

${ }^{53}$ Institute for Stroke and Dementia Research, University Hospital, LudwigMaximilians-University, Munich 
${ }^{54}$ Munich Cluster for Systems Neurology (SyNergy), Munich, Germany

${ }^{55}$ Deutsches Zentrum für Neurodegenerative Erkrankungen (DZNE), Munich, Germany

${ }^{56}$ INSERM U1219 Bordeaux Population Health Research Center, University of Bordeaux, Bordeaux, France

${ }^{57}$ Department of Neurology, Institute for Neurodegenerative Disease, Bordeaux University Hospital, Bordeaux, France

${ }^{58}$ Department of Clinical Neuroscience at Institute of Neuroscience and Physiology, University of Gothenburg, Sweden

${ }^{59}$ Univ. Lille, Inserm, Centre Hosp. Univ Lille, Institut Pasteur de Lille, UMR1167 LabEx DISTALZ -RID-AGE - Risk factors and molecular determinants of agingrelated diseases, F-59000 Lille, France

${ }^{60}$ Departments of Neurology and Public Health Sciences, University of Virginia School of Medicine, Charlottesville, VA, USA

${ }^{61}$ Department of Neurology, Faculty of Medicine, Jagiellonian University Medical College, ul. Botaniczna 3, 31-503, Krakow, Poland

${ }^{62}$ Department of Neurosurgery, Helsinki University Hospital, University of Helsinki, Finland

${ }^{63}$ Clinical Neurosciences, University of Helsinki, Topeliuksenkatu 5, 00260, Helsinki, Finland

${ }^{64}$ Neurosurgery NeuroCenter Kuopio University Hospital Kuopio Finland

${ }^{65}$ Institute of Clinical Medicine Faculty of Health Sciences University of Eastern Finland Kuopio Finland

${ }^{66}$ University of Cincinnati College of Medicine, Cincinnati, OH, USA

${ }^{1}$ Research and Communication Unit for Musculoskeletal Health (FORMI), Department of Research, Innovation and Education, Division of Clinical Neuroscience, Oslo University Hospital, Oslo, Norway

${ }^{2}$ Department of Neurology, Oslo University Hospital, Oslo, Norway

${ }^{3}$ K. G. Jebsen Center for Genetic Epidemiology, Department of Public Health and Nursing, Faculty of Medicine and Health Sciences, Norwegian University of Science and Technology, Trondheim, Norway

${ }^{4}$ Department of Clinical and Molecular Medicine, Norwegian University of Science and Technology, Trondheim, Norway

${ }^{5}$ Stroke Unit, Department of Neurology, Oslo University Hospital, Norway

${ }^{6}$ Research and Development, The Norwegian Air Ambulance Foundation, Norway

${ }^{7}$ Department of Neurology, Akershus University Hospital, Lørenskog, Norway 
${ }^{8}$ Department of Research, Innovation and Education, Division of Clinical Neuroscience, Oslo University Hospital, Oslo, Norway

${ }^{9}$ Department of Neuromedicine and Movement Science, Faculty of Medicine and Health Sciences, Norwegian University of Science and Technology (NTNU), Trondheim, Norway

${ }^{10}$ Stroke Unit, Department of Internal Medicine, St. Olavs Hospital, Trondheim University Hospital, Trondheim, Norway

${ }^{11}$ Department of Internal Medicine, Division of Cardiovascular Medicine, University of Michigan, Ann Arbor, 48109, MI, USA

${ }^{12}$ Department of Epidemiology Research, Statens Serum Institut, Copenhagen, Denmark

${ }^{13}$ Department of Neuroscience and Movement science, Norwegian University of Science and Technology, Trondheim, Norway

${ }^{14}$ Clinical Resarch Unit, St. Olavs Hospital, Trondheim, Norway

${ }^{15}$ Center for Statistical Genetics, Department of Biostatistics, University of Michigan, Ann Arbor, 48109, MI, USA

${ }^{16}$ HUNT Research Center, Department of Public Health and Nursing, Faculty of Medicine and Health Sciences, Norwegian University of Science and Technology, Trondheim, Norway

${ }^{17}$ Department of Computational Medicine and Bioinformatics, University of Michigan, Ann Arbor, MI, USA

${ }^{18}$ Analytic and Translational Genetics Unit, Massachusetts General Hospital, Boston, Massachusetts, USA

${ }^{1}$ China National Center for Food Safety Risk Assessment, Beijing, China

${ }^{2}$ Clinical Trial Service Unit \& Epidemiological Studies Unit (CTSU), Nuffield Department of Population Health, University of Oxford, United Kingdom

${ }^{3}$ Medical Research Council Population Health Research Unit (MRC PHRU), Nuffield Department of Population Health, University of Oxford, United Kingdom

${ }^{4}$ Chinese Academy of Medical Sciences, Beijing, China

${ }^{5}$ Department of Epidemiology, School of Public Health, Peking University Health Science Center, Beijing, China

${ }^{6}$ Centre for Disease Control and Prevention, Qingdao Province, Shandong, China

${ }^{7}$ Licang Centre for Disease Control and Prevention, Qingdao Province, Shandong, China

${ }^{8}$ Centre for Disease Control and Prevention, Heilongjiang Province, Harbin, China

${ }^{9}$ Nangang Centre for Disease Control and Prevention, Heilongjiang Province, Harbin, China 
${ }^{10}$ Centre for Disease Control and Prevention, Hainan Province, Haikou, China

${ }^{11}$ Meilan Centre for Disease Control and Prevention, Hainan Province, Haikou, China

${ }^{12}$ Centre for Disease Control and Prevention, Jiangsu Province, Nanjing, China

${ }^{13}$ Suzhou Centre for Disease Control and Prevention, Jiangsu Province, Suzhou, China

${ }^{14}$ Wuzhong Centre for Disease Control and Prevention, Jiangsu Province, Suzhou, China

${ }^{15}$ Centre for Disease Control and Prevention, Guangxi Province, Nanning, China

${ }^{16}$ Liuzhou Centre for Disease Control and Prevention, Guangxi Province, Liuzhou, China

${ }^{17}$ Centre for Disease Control and Prevention, Sichuan Province, Chengdu, China

${ }^{18}$ Pengzhou Centre for Disease Control and Prevention, Sichuan Province, Pengzhou, China

${ }^{19}$ Centre for Disease Control and Prevention, Gansu Province, Lanzhou, China

${ }^{20}$ Maiji Centre for Disease Control and Prevention, Gansu Province, Tianshui, China

${ }^{21}$ Centre for Disease Control and Prevention, Henan Province, Zhengzhou, China

${ }^{22}$ Huixian Centre for Disease Control and Prevention, Henan Province, Huixian, China

${ }^{23}$ Centre for Disease Control and Prevention, Zhejiang Province, Hangzhou, China

${ }^{24}$ Tongxiang Centre for Disease Control and Prevention, Zhejiang Province, Tongxiang, China

${ }^{25}$ Centre for Disease Control and Prevention, Hunan Province, Changsha, China

${ }^{26}$ Liuyang Centre for Disease Control and Prevention, Hunan Province, Liuyang, China

\section{Acknowledgements}

This research has been conducted using the UK Biobank Resource under application number 2532. We acknowledge R. McLaughlin for the advice on population-based heritability analysis. We acknowledge M. Gunel and K. Yasuno for their help with genotyping DNA samples of the Utrecht 1, Finland, and @ neurIST cohorts. The authors thank the staff and participants of all CADISP centers for their important contributions. The authors acknowledge the contribution of participants, project staff, and China National Centre for Disease Control and Prevention (CDC) and its regional offices to the China Kadoorie Biobank. China's National Health Insurance provided electronic linkage to all hospital treatments. The authors acknowledge K. Jebsen for genotyping quality control and imputation of the HUNT Study.

For providing clinical information and biological samples collected during the @ neurIST project, we thank: J. Macho, T. Dóczi, J. Byrne, P. Summers, R. Risselada, M. Sturkenboom, U. Patel, S. Coley, A. Waterworth, D. Rüfenacht, C. Proust, and F. Cambien. 
We acknowledge the support from the Netherlands Cardiovascular Research Initiative: An initiative with support of the Dutch Heart Foundation, CVON2015-08 ERASE.

This project has received funding from the European Research Council (ERC) under the European Union's Horizon 2020 research and innovation programme (grant agreement No. 852173).

This project has received funding from the European Research Council (ERC) under the European Union's Horizon 2020 research and innovation programme (grant agreement No. 772376 - EScORIAL).

BioBank Japan project was supported by the Ministry of Education, Culture, Sports, Sciences, and Technology of the Japanese government and the Japan Agency for Medical Research and Development (19km0605001).

The CADISP study has been supported by INSERM, Lille 2 University, Institut Pasteur de Lille and Lille University Hospital and received funding from the European Regional Development Fund (FEDER funds) and Région Nord-Pas-de-Calais in the framework of Contrat de Projets Etat-Region 2007-2013 Région Nord-Pasde-Calais (grant 09120030), Centre National de Génotypage, the Emil Aaltonen Foundation, the Paavo Ilmari Ahvenainen Foundation, the Helsinki University Central Hospital Research Fund, the Helsinki University Medical Foundation, the Päivikki and Sakari Sohlberg Foundation, the Aarne Koskelo Foundation, the Maire Taponen Foundation, the Aarne and Aili Turunen Foundation, the Lilly Foundation, the Alfred Kordelin Foundation, the Finnish Medical Foundation, the Orion Farmos Research Foundation, the Maud Kuistila Foundation, the Finnish Brain Foundation, the Biomedicum Helsinki Foundation, Projet Hospitalier de Recherche Clinique Régional, Fondation de France, Génopôle de Lille, Adrinord, the Basel Stroke Funds, the Käthe-Zingg-Schwichtenberg-Fonds of the Swiss Academy of Medical Sciences and the Swiss Heart Foundation.

S.D. has received funding from the French National Funding Agency (ANR), the European Research Council (ERC) under the European Union's Horizon 2020 research and innovation programme (grant agreement No 640643).

BioBank Japan project was supported by the Ministry of Education, Culture, Sports, Sciences, and Technology of the Japanese government and the Japan Agency for Medical Research and Development (19km0605001).

J.P. was supported by Jagiellonian University Medical College (JUMC) grant K/ZDS/001456.

China Kadoorie Biobank was supported as follows: Baseline survey and first re-survey: Hong Kong Kadoorie Charitable Foundation; long-term follow-up: UK Wellcome Trust (202922/Z/16/Z, 104085/Z/14/Z, 088158/Z/09/Z), National Natural Science Foundation of China (81390540, 81390541, 81390544), and National Key Research and Development Program of China (2016YFC 0900500, 0900501, 0900504, 1303904). DNA extraction and genotyping: GlaxoSmithKline, UK Medical Research Council (MC_PC_13049, MC-PC-14135). Core funding to the Clinical Trial Service Unit and Epidemiological Studies Unit at Oxford University was provided by The British Heart Foundation, UK MRC, and Cancer Research UK.

S.Z. and G.A.R. received funding from Canadian Institutes of Health Research (CIHR). This project has received funding from the European Union's Horizon 2020 research and innovation programme (No. 666881), SVDs@ arget (to M.D.) and No. 667375, CoSTREAM (Common Mechanisms and Pathways in Stroke and Alzheimer's Disease; to M.D.); the DFG (Deutsche Forschungsgemeinschaft) as part of the Munich Cluster for Systems Neurology (EXC 2145 SyNergy-ID 390857198) and the CRC 1123 (B3, to M.D.); the Corona Foundation (to M.D.); the Fondation Leducq (Transatlantic Network of Excellence on the Pathogenesis of Small Vessel Disease of the Brain, to M.D.); the e:Med program (e:AtheroSysMed, to M.D.); and the FP7/2007-2103 European Union project CVgenes@target (grant agreement No. Health-F2-2013-601456, to M.D.). K.R. is funded by the Health Data Research UK (HDRUK) fellowship MR/S004130/1. C.L.M.S. was funded by the UK Biobank, Health Data Research UK, and Scottish Funding Council.

I.C.H. received funding from the Alzheimer Research UK and Dunhill Medical Trust Foundation.

J.P.B. and D.W. were supported by NIH Funding.

D.J.W. and V.S.A. received funding support from The Stroke Association.

D.J.W. and H.H. received funding for genotyping from the National Institute for Health

Research University College London Hospitals Biomedical Research Centre.

The Nord-Trøndelag Health Study (HUNT Study) is a collaboration between the HUNT Research Centre, Faculty of Medicine at the Norwegian University of Science and Technology (NTNU), the Norwegian Institute of Public 
Health and the Nord- Trøndelag County Council. The genotyping was financed by the National Institute of health (NIH), University of Michigan, The Norwegian Research council, and Central Norway Regional Health Authority and the Faculty of Medicine and Health Sciences, Norwegian University of Science and Technology (NTNU).

P.B. and C.M.F. were supported by EU commission FP6 - IST - 027703 @ neurIST-Integrated biomedical informatics for the management of cerebral aneurysms.

P.B., S.M., S.H., S.S., J.D., and O.M. were supported by the Grant MRD 2014/261 from the Swiss SystemsX.ch initiative and evaluated by the Swiss National Science Foundation (AneuX project).

\section{Data availability statement}

Summary statistics for the Stage 1 and Stage 2 GWAS meta-analyses, the SAH-only, and uIA-only GWAS, and a meta-analysis consisting of only East Asian samples, including effective sample size per SNP, can be accessed upon publication using doi: https://doi.org/ 10.6084/m9.figshare.11303372. And through the Cerebrovascular Disease Knowledge Portal: http://www.cerebrovascularportal.org. Detailed information on access of publicly available data is given in the Life Sciences Reporting Summary.

\section{References}

1. Vlak MH, Algra A, Brandenburg R, Rinkel GJ. Prevalence of unruptured intracranial aneurysms, with emphasis on sex, age, comorbidity, country, and time period: a systematic review and metaanalysis. Lancet Neurol. 2011; 10: 626-36. [PubMed: 21641282]

2. Nieuwkamp DJ, et al. Changes in case fatality of aneurysmal subarachnoid haemorrhage over time, according to age, sex, and region: a meta-analysis. Lancet Neurol. 2009; 8: 635-42. [PubMed: 19501022]

3. Korja M, et al. Genetic epidemiology of spontaneous subarachnoid hemorrhage: Nordic Twin Study. Stroke. 2010; 41: 2458-62. [PubMed: 20847318]

4. Kurki MI, et al. High risk population isolate reveals low frequency variants predisposing to intracranial aneurysms. PLoS Genet. 2014; 10: e1004134. [PubMed: 24497844]

5. Yasuno K, et al. Common variant near the endothelin receptor type A (EDNRA) gene is associated with intracranial aneurysm risk. Proc Natl Acad Sci U S A. 2011; 108: 19707-12. [PubMed: 22106312]

6. Yan J, et al. Genetic study of intracranial aneurysms. Stroke. 2015; 46: 620-6. [PubMed: 25649796]

7. Santiago-Sim T, et al. THSD1 (Thrombospondin Type 1 Domain Containing Protein 1) Mutation in the Pathogenesis of Intracranial Aneurysm and Subarachnoid Hemorrhage. Stroke. 2016; 47: 3005-13. [PubMed: 27895300]

8. Bourcier R, et al. Rare Coding Variants in ANGPTL6 Are Associated with Familial Forms of Intracranial Aneurysm. Am J Hum Genet. 2018; 102: 133-141. [PubMed: 29304371]

9. Lorenzo-Betancor O, et al. PCNT point mutations and familial intracranial aneurysms. Neurology. 2018; 91: e2170-e2181. [PubMed: 30413633]

10. Zhou S, et al. RNF213 Is Associated with Intracranial Aneurysms in the French-Canadian Population. Am J Hum Genet. 2016; 99: 1072-1085. [PubMed: 27745834]

11. Hussain I, Duffis EJ, Gandhi CD, Prestigiacomo CJ. Genome-wide association studies of intracranial aneurysms: an update. Stroke. 2013; 44: 2670-5. [PubMed: 23908070]

12. Foroud T, et al. Genome-wide association study of intracranial aneurysms confirms role of Anril and SOX17 in disease risk. Stroke. 2012; 43: 2846-52. [PubMed: 22961961]

13. Yasuno K, et al. Genome-wide association study of intracranial aneurysm identifies three new risk loci. Nat Genet. 2010; 42: 420-5. [PubMed: 20364137]

14. Zhou W, et al. Efficiently controlling for case-control imbalance and sample relatedness in largescale genetic association studies. Nat Genet. 2018; 50: 1335-1341. [PubMed: 30104761]

15. Yang J, et al. Conditional and joint multiple-SNP analysis of GWAS summary statistics identifies additional variants influencing complex traits. Nat Genet. 2012; 44: 369-75. [PubMed: 22426310] 
16. Zhu ZH, et al. Causal associations between risk factors and common diseases inferred from GWAS summary data. Nature Communications. 9: 2018;

17. Tobacco Genetics Consortium. Genome-wide meta-analyses identify multiple loci associated with smoking behavior. Nat Genet. 2010; 42: 441-7. [PubMed: 20418890]

18. Evangelou E, et al. Genetic analysis of over 1 million people identifies 535 new loci associated with blood pressure traits. Nat Genet. 2018; 50: 1412-1425. [PubMed: 30224653]

19. Lee $S$, et al. Deficiency of endothelium-specific transcription factor Sox 17 induces intracranial aneurysm. Circulation. 2015; 131: 995-1005. [PubMed: 25596186]

20. Laarman MD, et al. Chromatin Conformation Links Putative Enhancers in Intracranial AneurysmAssociated Regions to Potential Candidate Genes. J Am Heart Assoc. 2019; 8: e011201. [PubMed: 30994044]

21. Giri A, et al. Trans-ethnic association study of blood pressure determinants in over 750,000 individuals. Nat Genet. 2019; 51: 51-62. [PubMed: 30578418]

22. Kichaev G, et al. Leveraging Polygenic Functional Enrichment to Improve GWAS Power. Am J Hum Genet. 2019; 104: 65-75. [PubMed: 30595370]

23. Takeuchi F, et al. Interethnic analyses of blood pressure loci in populations of East Asian and European descent. Nat Commun. 2018; 9

24. Hoffmann TJ, et al. Genome-wide association analyses using electronic health records identify new loci influencing blood pressure variation. Nat Genet. 2017; 49: 54-64. [PubMed: 27841878]

25. Huang L, et al. A missense variant in FGD6 confers increased risk of polypoidal choroidal vasculopathy. Nat Genet. 2016; 48: 640-7. [PubMed: 27089177]

26. Romanoski CE, et al. Systems genetics analysis of gene-by-environment interactions in human cells. Am J Hum Genet. 2010; 86: 399-410. [PubMed: 20170901]

27. Haasdijk RA, et al. THSD1 preserves vascular integrity and protects against intraplaque haemorrhaging in ApoE-/- mice. Cardiovasc Res. 2016; 110: 129-39. [PubMed: 26822228]

28. Camacho Leal Mdel P, et al. p130Cas/BCAR1 scaffold protein in tissue homeostasis and pathogenesis. Gene. 2015; 562: 1-7. [PubMed: 25727852]

29. Nedeljkovic I, et al. Understanding the role of the chromosome 15q25.1 in COPD through epigenetics and transcriptomics. Eur J Hum Genet. 2018; 26: 709-722. [PubMed: 29422661]

30. David SP, et al. Genome-wide meta-analyses of smoking behaviors in African Americans. Transl Psychiatry. 2012; 2: e119.

31. Liu M, et al. Association studies of up to 1.2 million individuals yield new insights into the genetic etiology of tobacco and alcohol use. Nat Genet. 2019; 51: 237-244. [PubMed: 30643251]

32. Lutz SM, et al. A genome-wide association study identifies risk loci for spirometric measures among smokers of European and African ancestry. BMC Genet. 2015; 16: 138. [PubMed: 26634245]

33. Bulik-Sullivan BK, et al. LD Score regression distinguishes confounding from polygenicity in genome-wide association studies. Nat Genet. 2015; 47: 291-5. [PubMed: 25642630]

34. Speed D, Balding DJ. SumHer better estimates the SNP heritability of complex traits from summary statistics. Nat Genet. 2019; 51: 277-284. [PubMed: 30510236]

35. Watanabe K, et al. A global overview of pleiotropy and genetic architecture in complex traits. Nat Genet. 2019; 51: 1339-1348. [PubMed: 31427789]

36. Skene NG, et al. Genetic identification of brain cell types underlying schizophrenia. Nat Genet. 2018; 50: 825-833. [PubMed: 29785013]

37. He L, et al. Single-cell RNA sequencing of mouse brain and lung vascular and vessel-associated cell types. Sci Data. 2018; 5

38. Backes D, Rinkel GJ, Laban KG, Algra A, Vergouwen MD. Patient- and Aneurysm-Specific Risk Factors for Intracranial Aneurysm Growth: A Systematic Review and Meta-Analysis. Stroke. 2016; 47: 951-7. [PubMed: 26906920]

39. Muller TB, Vik A, Romundstad PR, Sandvei MS. Risk Factors for Unruptured Intracranial Aneurysms and Subarachnoid Hemorrhage in a Prospective Population-Based Study. Stroke. 2019; 50: 2952-2955. [PubMed: 31370767] 
40. Algra AM, Klijn CJ, Helmerhorst FM, Algra A, Rinkel GJ. Female risk factors for subarachnoid hemorrhage: a systematic review. Neurology. 2012; 79: 1230-6. [PubMed: 22955127]

41. Zheng J, et al. LD Hub: a centralized database and web interface to perform LD score regression that maximizes the potential of summary level GWAS data for SNP heritability and genetic correlation analysis. Bioinformatics. 2017; 33: 272-279. [PubMed: 27663502]

42. Malik R, et al. Multiancestry genome-wide association study of 520,000 subjects identifies 32 loci associated with stroke and stroke subtypes. Nat Genet. 2018; 50: 524-537. [PubMed: 29531354]

43. Weinsheimer $S$, et al. Genome-wide association study of sporadic brain arteriovenous malformations. J Neurol Neurosurg Psychiatry. 2016; 87: 916-23. [PubMed: 26818729]

44. Debette $\mathrm{S}$, et al. Common variation in PHACTR1 is associated with susceptibility to cervical artery dissection. Nat Genet. 2015; 47: 78-83. [PubMed: 25420145]

45. Jones GT, et al. Meta-Analysis of Genome-Wide Association Studies for Abdominal Aortic Aneurysm Identifies Four New Disease-Specific Risk Loci. Circ Res. 2017; 120: 341-353. [PubMed: 27899403]

46. Hankey GJ. Stroke. Lancet. 2017; 389: 641-654. [PubMed: 27637676]

47. An SJ, Kim TJ, Yoon BW. Epidemiology, Risk Factors, and Clinical Features of Intracerebral Hemorrhage: An Update. J Stroke. 2017; 19: 3-10. [PubMed: 28178408]

48. Gaspar HA, Breen G. Drug enrichment and discovery from schizophrenia genome-wide association results: an analysis and visualisation approach. Sci Rep. 2017; 7

49. Rogawski MA, Loscher W. The neurobiology of antiepileptic drugs. Nat Rev Neurosci. 2004; 5 : 553-64. [PubMed: 15208697]

50. Lindbohm JV, Kaprio J, Jousilahti P, Salomaa V, Korja M. Sex, Smoking, and Risk for Subarachnoid Hemorrhage. Stroke. 2016; 47: 1975-81. [PubMed: 27444257]

51. Vlak MH, Rinkel GJ, Greebe P, Algra A. Risk of rupture of an intracranial aneurysm based on patient characteristics: a case-control study. Stroke. 2013; 44: 1256-9. [PubMed: 23520239]

52. Juvela S, Poussa K, Porras M. Factors affecting formation and growth of intracranial aneurysms: a long-term follow-up study. Stroke. 2001; 32: 485-91. [PubMed: 11157187]

53. Kobeissi E, Hibino M, Pan H, Aune D. Blood pressure, hypertension and the risk of abdominal aortic aneurysms: a systematic review and meta-analysis of cohort studies. Eur J Epidemiol. 2019; 34: 547-555. [PubMed: 30903463]

54. Cheng J, et al. Ion Channels and Vascular Diseases. Arterioscler Thromb Vasc Biol. 2019; 39: e146-e156. [PubMed: 31017824]

55. Bulley S, et al. Arterial smooth muscle cell PKD2 (TRPP1) channels regulate systemic blood pressure. Elife. 2018; 7

56. Perrone RD, Malek AM, Watnick T. Vascular complications in autosomal dominant polycystic kidney disease. Nat Rev Nephrol. 2015; 11: 589-98. [PubMed: 26260542]

57. Willer CJ, Li Y, Abecasis GR. METAL: fast and efficient meta-analysis of genomewide association scans. Bioinformatics. 2010; 26: 2190-1. [PubMed: 20616382]

58. Hormozdiari F, et al. Colocalization of GWAS and eQTL Signals Detects Target Genes. Am J Hum Genet. 2016; 99: 1245-1260. [PubMed: 27866706]

59. Gusev A, et al. Integrative approaches for large-scale transcriptome-wide association studies. Nat Genet. 2016; 48: 245-52. [PubMed: 26854917]

60. Zhu Z, et al. Integration of summary data from GWAS and eQTL studies predicts complex trait gene targets. Nat Genet. 2016; 48: 481-7. [PubMed: 27019110]

61. Finucane HK, et al. Partitioning heritability by functional annotation using genome-wide association summary statistics. Nat Genet. 2015; 47: 1228-35. [PubMed: 26414678]

62. Iotchkova V, et al. GARFIELD classifies disease-relevant genomic features through integration of functional annotations with association signals. Nat Genet. 2019; 51: 343-353. [PubMed: 30692680]

63. Woo D, et al. Meta-analysis of genome-wide association studies identifies 1q22 as a susceptibility locus for intracerebral hemorrhage. Am J Hum Genet. 2014; 94: 511-21. [PubMed: 24656865] 
64. Brown BC, Asian Genetic Epidemiology Network Type 2 Diabetes. Ye C, Price CJ, A.L, Zaitlen N. Transethnic Genetic-Correlation Estimates from Summary Statistics. Am J Hum Genet. 2016; 99: 76-88. [PubMed: 27321947]

65. Mootha VK, et al. PGC-1alpha-responsive genes involved in oxidative phosphorylation are coordinately downregulated in human diabetes. Nat Genet. 2003; 34: 267-73. [PubMed: 12808457]

66. Subramanian A, et al. Gene set enrichment analysis: a knowledge-based approach for interpreting genome-wide expression profiles. Proc Natl Acad Sci U S A. 2005; 102: 15545-50. [PubMed: 16199517] 


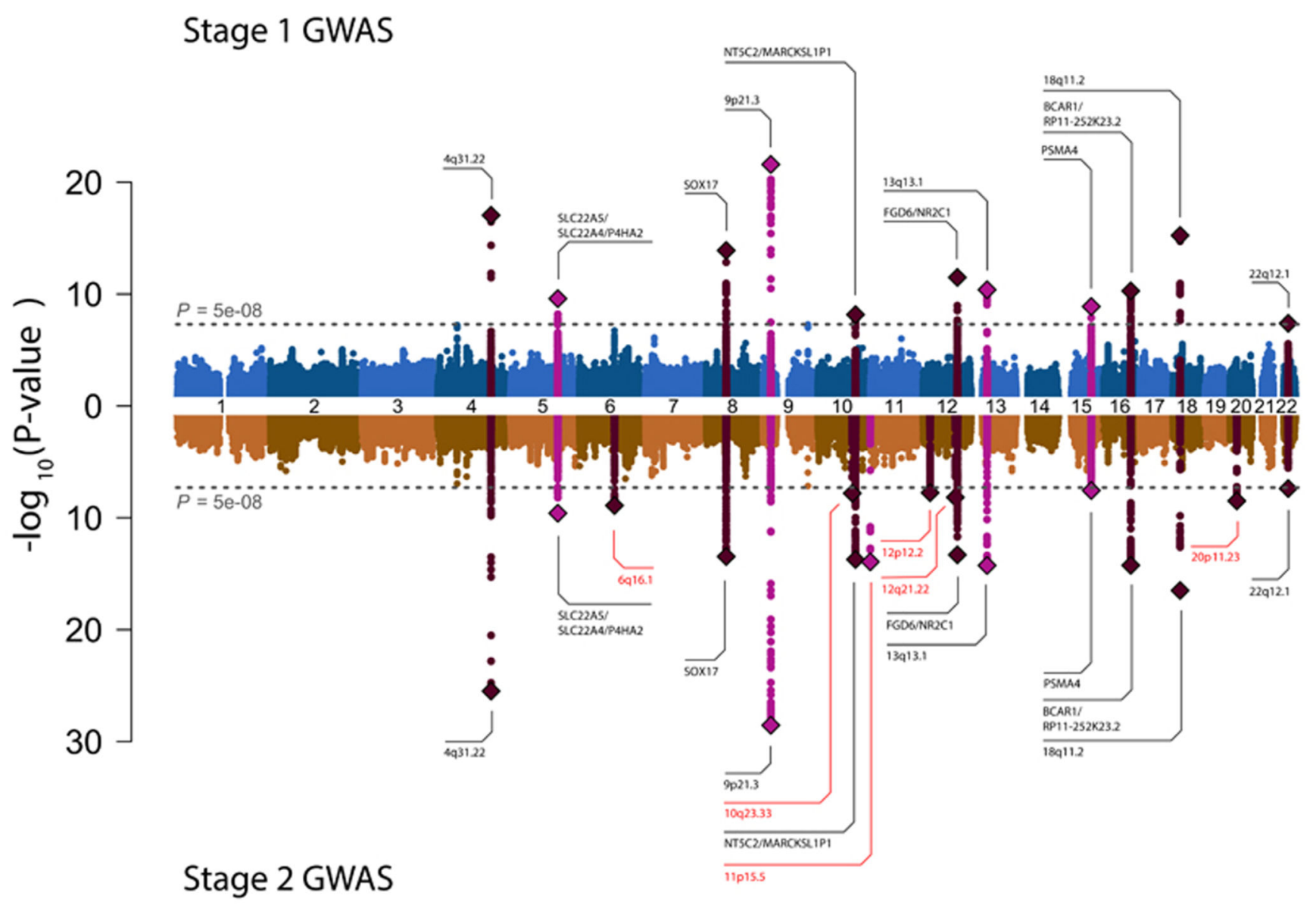

Figure 1. GWAS meta-analysis association results.

SAIGE logistic mixed model association P-values of the Stage 1 (upwards direction) and Stage 2 (downwards direction) GWAS meta-analyses. The horizontal axis indicates chromosomal position. The vertical axis indicates $-\log _{10}(\mathrm{P}$-value $)$ of the association. The dotted lines indicate the genome-wide significance threshold of $\mathrm{P}=5 \cdot 10^{-8}$. Lead SNPs of each locus are highlighted with a diamond, and SNPs in close proximity $( \pm 500 \mathrm{Kbp})$ are colored in pink or purple, depending on chromosome index parity. Labels are gene or locus names annotated using SMR, eCAVIAR and TWAS, or prior information of intracranial aneurysm-associated genes. Labels or loci identified only in the Stage 2 GWAS are shown in red. 
a

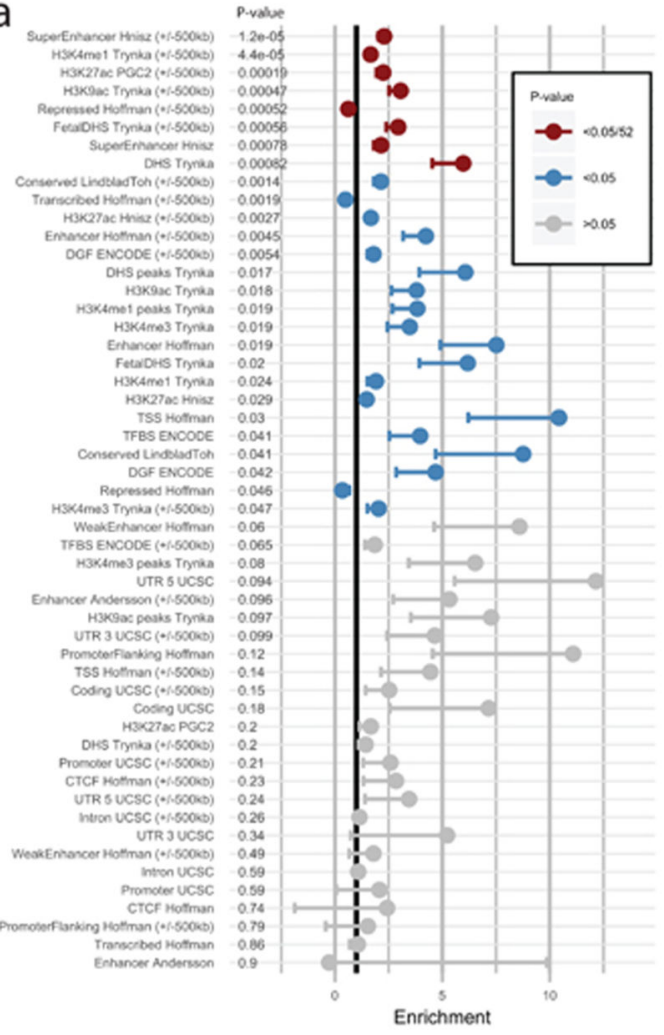

b

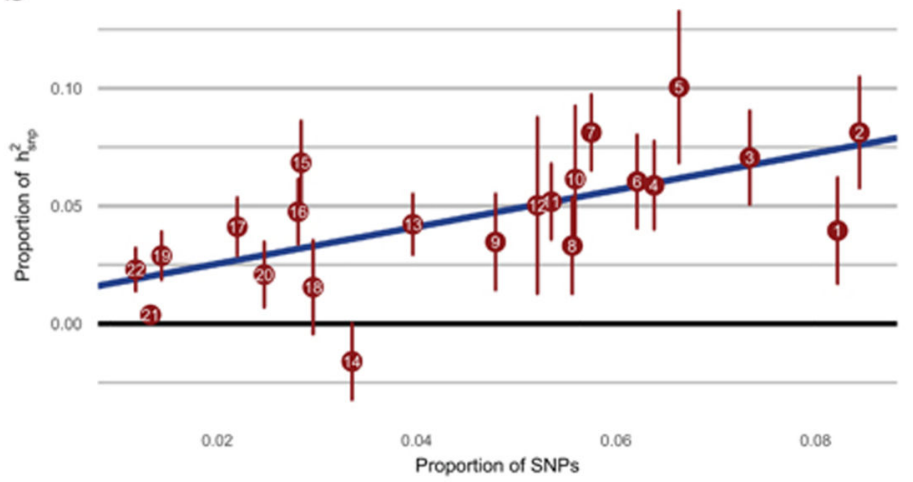

C

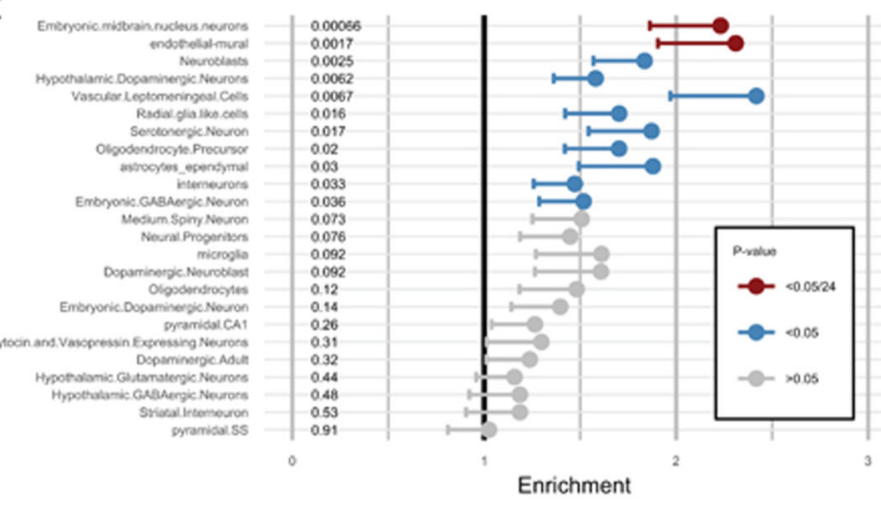

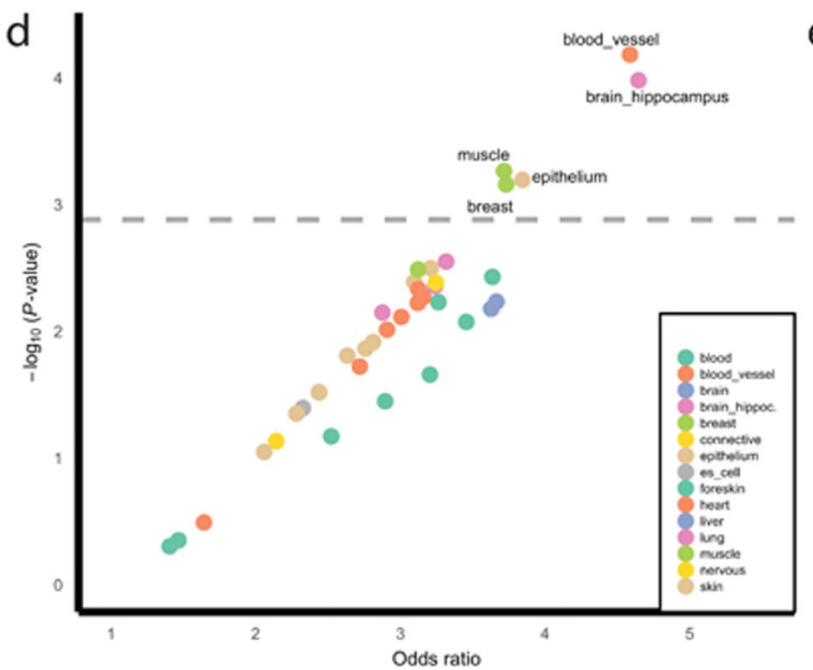

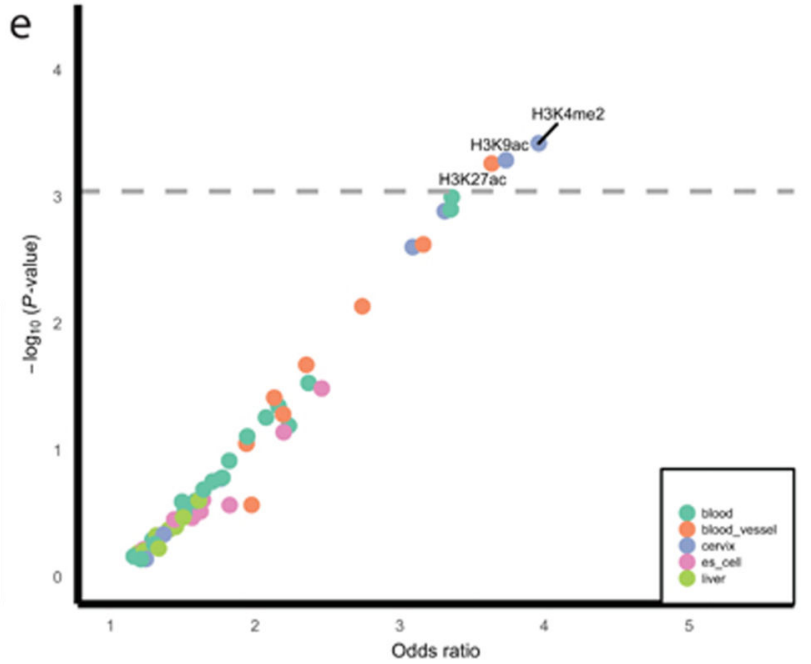

Figure 2. Heritability and functional enrichment analyses.

a) Partitioned LDSR enrichment of regulatory elements. Labels indicate type of regulatory element or histone mark. On the horizontal axis, the enrichment is shown. Enrichment=1 indicates no enrichment. Statistical significance was defined as P-value below 0.05 divided by the number of annotations (52). Effective $\mathrm{N}$ varies per SNP (see data availability statement). Points are estimates and error bars denote one standard error in the direction of no effect. Statistics derived from two-sided, weighted linear regression. No P-value adjustment. b) Partitioned LDSR heritability analysis per chromosome. On the horizontal 
axis the proportion of SNPs on each chromosome is shown. On the vertical axis the proportion of SNP-based heritability. The linear regression line is shown in blue. Data is presented as point estimate \pm standard error. Statistics the same as used for 2a. c) Partitioned LDSR enrichment analysis of scRNAseq brain cell types. Format and statistics are the same as used for 2a. d) GARFIELD analysis of tissues. On the horizontal axis, the enrichment of annotations is shown; on the vertical axis, the corresponding $-\log _{10}(\mathrm{P}$-value $)$. Dashed line indicates the significance threshold of $\mathrm{P}=0.05$ divided by the number of annotations. Odds ratios are derived by logistic regression. P-values are unadjusted, derived from two-sided test. e) GARFIELD analysis of regulatory regions defined by histone modifications. Format and statistics are the same as used for $2 \mathrm{~d}$ ). 
a

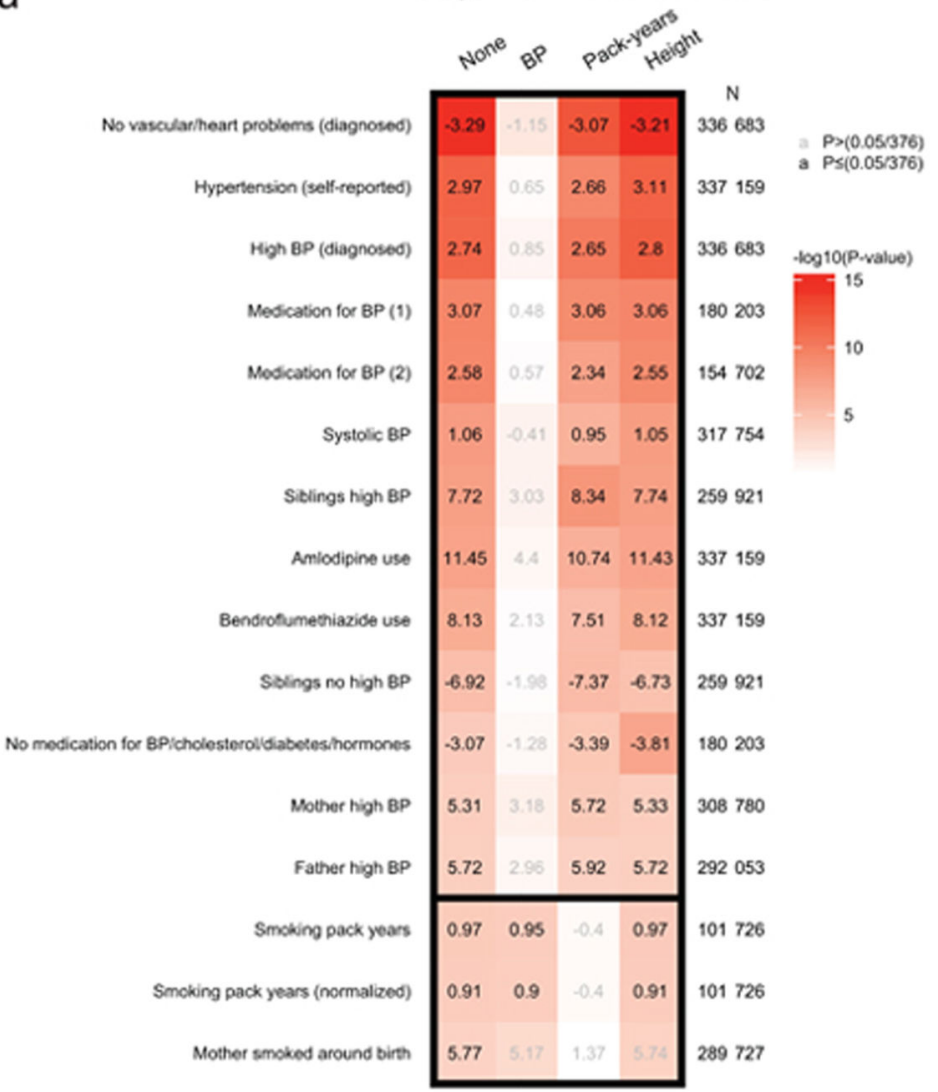

b

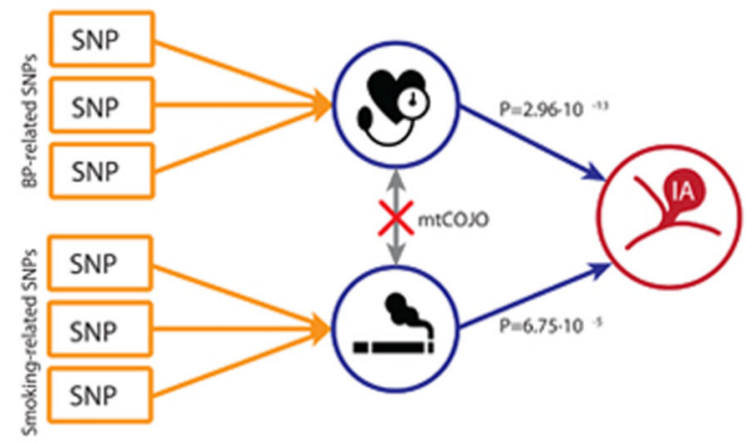

C

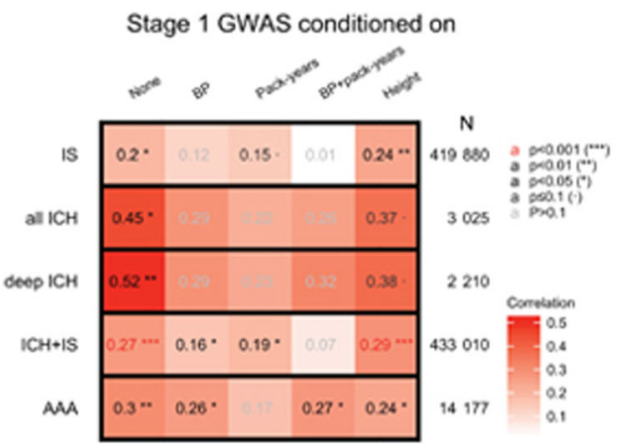

Figure 3. Cross-trait analyses.

a) GSMR analysis of UK Biobank predictors on the Stage 1 intracranial aneurysm GWAS, conditioned on traits depicted by column labels with mtCOJO. Numeric values are the GSMR effect sizes. The top 13 traits are blood pressure-related traits. The bottom three traits are smoking-related. Statistical significance was defined as P-value below 0.05 divided by the number of traits that passed quality control (376). Square fill colors indicate $-\log _{10}(\mathrm{P}$-value $)$ of the GSMR effect. All 16 traits that pass the multiple testing threshold for significance in the unconditioned analysis are shown. BP: blood pressure. Presented N is sample size in UK Biobank GWAS. For intracranial aneurysms, effective $\mathrm{N}$ per SNP was used. P-values from two-sided linear regression, unadjusted. b) Causality diagram further explaining the analyses of 2a: GSMR analysis showed that genetic risk for smoking and BP are causative of intracranial aneurysms. Using mtCOJO, it was found that the genetic factors associated with BP and smoking cause intracranial aneurysms through independent mechanisms. Statistics the same as used for 3a. BP N=317,754 samples, smoking N=101,726 samples. c) Genetic correlation analysis with LDSR. Genetic correlation estimates are indicated by color and numeric value. Axis labels on the left denote the trait correlated with intracranial aneurysms. Labels on the top denote the trait for which the Stage 1 intracranial aneurysm GWAS was conditioned using mtCOJO. More details in Supplementary Table 3. Presented $\mathrm{N}$ is effective sample size for trait on the left. Except for 
IS and ICH+IS, where an N per SNP was used and average $\mathrm{N}$ is shown. IS: ischemic stroke. ICH: intracerebral hemorrhage. AAA: abdominal aortic aneurysm. 
Table 1

Lead associations of genome-wide significant risk loci.

Association statistics were derived by SAIGE logistic mixed model. P-values are unadjusted from a two-sided test. Risk loci reaching genome-wide significant threshold $\left(\mathrm{P}<5 \cdot 10^{-8}\right)$ in the Stage 2 GWAS of European and East Asian ancestry individuals are shown. Chr: Chromosome. Position: basepair position on GRCh37. EA: Effect allele. OA: Other allele. Stage 1: European ancestry only GWAS meta-analysis. East Asian: subset of samples from Japan and China. Stage 2: meta-analysis of European ancestry and East Asian data. EAF: Effect allele frequency. SE: Standard error of beta. Annotated genes are potentially causative genes identified using summary statistics based Mendelian randomization (SMR), eCAVIAR and transcriptome-wide association study (TWAS). Associated traits are cardiovascular traits and stroke risk factors with which the lead SNP is associated. CAD: Coronary artery disease. SBP: Systolic blood pressure. IS: Ischemic stroke. AAA: Abdominal aortic aneurysm. DBP: Diastolic blood pressure. CVD: Cardiovascular disease. COPD: Chronic obstructive pulmonary disease. †Known locus, described in Hussain et al (2013). *Another SNP in this locus ( $r^{2}>0.8$ with the Stage 2 lead SNP) has a lower P-value, due to differences in LD patterns between European and East Asian populations. For locus 15q25.1, another SNP in that locus reaches genome-wide significance in Stage 1. **For two SNPs, no East Asian association statistics could be obtained, because these SNPs are monomorphic in Japanese and Chinese populations (LDlink, https://ldlink.nci.nih.gov/).

\begin{tabular}{|c|c|c|c|c|c|c|c|c|c|c|c|c|}
\hline SNP & Locus & Chr & Position & EA & OA & Stage & EAF & beta & SE & P-value & $\begin{array}{c}\text { Annotated } \\
\text { genes }\end{array}$ & $\begin{array}{c}\text { Associated } \\
\text { traits }\end{array}$ \\
\hline \multirow{3}{*}{ rs6841581 } & \multirow{3}{*}{$4 \mathrm{q} 31.22 \dagger$} & \multirow{3}{*}{4} & \multirow{3}{*}{148401190} & \multirow{3}{*}{ A } & \multirow{3}{*}{ G } & $\begin{array}{c}\text { Stage } \\
1\end{array}$ & 0.131 & -0.262 & 0.031 & $1.08 \cdot 10^{-17 *}$ & \multirow{3}{*}{ - } & \multirow{3}{*}{ CAD } \\
\hline & & & & & & $\begin{array}{c}\text { East } \\
\text { Asian }\end{array}$ & 0.297 & -0.181 & 0.028 & $6.55 \cdot 10^{-11}$ & & \\
\hline & & & & & & $\begin{array}{l}\text { Stage } \\
2\end{array}$ & 0.222 & -0.218 & 0.021 & $3.22 \cdot 10^{-26}$ & & \\
\hline \multirow{3}{*}{ rs4705938 } & \multirow{3}{*}{$5 q 31.1$} & \multirow{3}{*}{5} & \multirow{3}{*}{131694077} & \multirow{3}{*}{$\mathrm{T}$} & \multirow{3}{*}{ C } & $\begin{array}{c}\text { Stage } \\
1\end{array}$ & 0.549 & 0.120 & 0.019 & $2.55 \cdot 10^{-10}$ & \multirow{3}{*}{$\begin{array}{c}\text { SLC22A5/ } \\
\text { SLC22A4/ } \\
\text { P4HA2 }\end{array}$} & \multirow{3}{*}{$\begin{array}{l}\text { Lung } \\
\text { function }\end{array}$} \\
\hline & & & & & & $\begin{array}{c}\text { East } \\
\text { Asian }\end{array}$ & $N A$ & $N A$ & $N A$ & $N A$ ** & & \\
\hline & & & & & & $\begin{array}{c}\text { Stage } \\
2\end{array}$ & 0.549 & 0.120 & 0.019 & $2.55 \cdot 10^{-10}$ & & \\
\hline \multirow{3}{*}{ rs11153071 } & \multirow{3}{*}{$6 q 16.1$} & \multirow{3}{*}{6} & \multirow{3}{*}{97039741} & \multirow{3}{*}{ A } & \multirow{3}{*}{ G } & $\begin{array}{c}\text { Stage } \\
1\end{array}$ & 0.185 & 0.158 & 0.032 & $5.86 \cdot 10^{-7 *}$ & \multirow{3}{*}{ - } & \multirow{3}{*}{$\begin{array}{c}\text { SBP, } \\
\text { migraine, } \\
\text { sleep } \\
\text { quality }\end{array}$} \\
\hline & & & & & & $\begin{array}{c}\text { East } \\
\text { Asian }\end{array}$ & 0.113 & 0.143 & 0.041 & $5.29 \cdot 10^{-4}$ & & \\
\hline & & & & & & $\begin{array}{l}\text { Stage } \\
2\end{array}$ & 0.158 & 0.153 & 0.025 & $1.25 \cdot 10^{-9}$ & & \\
\hline \multirow{3}{*}{ rs62516550 } & \multirow{2}{*}{$8 \mathrm{q} 11.23 \dagger$} & \multirow{2}{*}{8} & \multirow{2}{*}{55467028} & \multirow{2}{*}{$\mathrm{T}$} & \multirow{2}{*}{$\mathrm{C}$} & $\begin{array}{c}\text { Stage } \\
1\end{array}$ & 0.389 & 0.169 & 0.023 & $1.44 \cdot 10^{-13 *}$ & \multirow{2}{*}{ SOX17 } & \multirow{2}{*}{-} \\
\hline & & & & & & $\begin{array}{c}\text { East } \\
\text { Asian }\end{array}$ & 0.087 & 0.102 & 0.049 & $3.70 \cdot 10^{-2}$ & & \\
\hline & & & & & & $\begin{array}{l}\text { Stage } \\
2\end{array}$ & 0.335 & 0.157 & 0.021 & $3.44 \cdot 10^{-14}$ & & \\
\hline \multirow{2}{*}{ rs 1537373} & \multirow{2}{*}{$9 \mathrm{p} 21.3 \dagger$} & \multirow{2}{*}{9} & & & & $\begin{array}{c}\text { Stage } \\
1\end{array}$ & 0.514 & -0.186 & 0.019 & $2.60 \cdot 10^{-22}$ & & \\
\hline & & & 22103341 & 1 & U & $\begin{array}{c}\text { East } \\
\text { Asian }\end{array}$ & 0.342 & -0.165 & 0.029 & $1.43 \cdot 10^{-8}$ & - & CAD \\
\hline
\end{tabular}




\begin{tabular}{|c|c|c|c|c|c|c|c|c|c|c|c|c|}
\hline SNP & Locus & Chr & Position & EA & OA & Stage & EAF & beta & SE & P-value & $\begin{array}{l}\text { Annotated } \\
\text { genes }\end{array}$ & $\begin{array}{c}\text { Associated } \\
\text { traits }\end{array}$ \\
\hline & & & & & & $\begin{array}{c}\text { Stage } \\
2\end{array}$ & 0.462 & -0.180 & 0.016 & $2.86 \cdot 10^{-29}$ & & \\
\hline \multirow{3}{*}{ rs 11187838} & \multirow{3}{*}{$10 \mathrm{q} 23.33$} & \multirow{3}{*}{10} & \multirow{3}{*}{96038686} & \multirow{3}{*}{ A } & \multirow{3}{*}{ G } & $\begin{array}{c}\text { Stage } \\
1\end{array}$ & 0.415 & -0.075 & 0.019 & $1.24 \cdot 10^{-4}$ & \multirow{3}{*}{-} & \multirow{3}{*}{$\begin{array}{c}\text { SBP, } \\
\text { migraine, } \\
\text { fat free } \\
\text { mass }\end{array}$} \\
\hline & & & & & & $\begin{array}{l}\text { East } \\
\text { Asian }\end{array}$ & 0.473 & -0.108 & 0.025 & $1.81 \cdot 10^{-5}$ & & \\
\hline & & & & & & $\begin{array}{l}\text { Stage } \\
2\end{array}$ & 0.436 & -0.087 & 0.015 & $1.55 \cdot 10^{-8}$ & & \\
\hline \multirow{3}{*}{ rs79780963 } & \multirow{3}{*}{$10 \mathrm{q} 24.32 \dagger$} & \multirow{3}{*}{10} & \multirow{3}{*}{104952499} & \multirow{3}{*}{$\mathrm{T}$} & \multirow{3}{*}{$\mathrm{C}$} & $\begin{array}{c}\text { Stage } \\
1\end{array}$ & 0.078 & -0.225 & 0.039 & $6.82 \cdot 10^{-9}$ & \multirow{3}{*}{$\begin{array}{c}\text { NTSC2/ } \\
\text { MARCKSL1P1 }\end{array}$} & \multirow{3}{*}{-} \\
\hline & & & & & & $\begin{array}{l}\text { East } \\
\text { Asian }\end{array}$ & 0.371 & -0.163 & 0.032 & $3.11 \cdot 10^{-7}$ & & \\
\hline & & & & & & $\begin{array}{c}\text { Stage } \\
2\end{array}$ & 0.254 & -0.188 & 0.025 & $2.34 \cdot 10^{-14}$ & & \\
\hline \multirow{3}{*}{ rs 2280543} & \multirow{3}{*}{$11 \mathrm{p} 15.5$} & \multirow{3}{*}{11} & \multirow{3}{*}{203788} & \multirow{3}{*}{$\mathrm{T}$} & \multirow{3}{*}{$\mathrm{C}$} & $\begin{array}{c}\text { Stage } \\
1\end{array}$ & 0.041 & 0.162 & 0.053 & $2.19 \cdot 10^{-3}$ & \multirow{3}{*}{ - } & \multirow{3}{*}{ - } \\
\hline & & & & & & $\begin{array}{l}\text { East } \\
\text { Asian }\end{array}$ & 0.131 & 0.277 & 0.038 & $2.87 \cdot 10^{-13}$ & & \\
\hline & & & & & & $\begin{array}{c}\text { Stage } \\
2\end{array}$ & 0.101 & 0.238 & 0.031 & $1.16 \cdot 10^{-14}$ & & \\
\hline \multirow{3}{*}{ rs11044991 } & \multirow{3}{*}{$12 \mathrm{p} 12.2$} & \multirow{3}{*}{12} & \multirow{3}{*}{20174364} & \multirow{3}{*}{ A } & \multirow{3}{*}{ G } & $\begin{array}{c}\text { Stage } \\
1\end{array}$ & 0.038 & -0.142 & 0.053 & $7.47 \cdot 10^{-3}$ & & \\
\hline & & & & & & $\begin{array}{c}\text { East } \\
\text { Asian }\end{array}$ & 0.476 & -0.125 & 0.025 & $6.74 \cdot 10^{-7}$ & - & $\begin{array}{l}\text { Mean } \\
\text { arterial } \\
\text { pressure }\end{array}$ \\
\hline & & & & & & $\begin{array}{l}\text { Stage } \\
2\end{array}$ & 0.395 & -0.128 & 0.023 & $1.74 \cdot 10^{-8}$ & & \\
\hline & & & & & & $\begin{array}{c}\text { Stage } \\
1\end{array}$ & 0.844 & 0.086 & 0.029 & $2.86 \cdot 10^{-3}$ & & SBI \\
\hline rs2681472 & $12 \mathrm{q} 21.33$ & 12 & 90008959 & A & G & $\begin{array}{l}\text { East } \\
\text { Asian }\end{array}$ & 0.629 & 0.131 & 0.026 & $5.29 \cdot 10^{-7}$ & - & $\begin{array}{l}\text { pulse } \\
\text { pressure, } \\
\text { CVD }\end{array}$ \\
\hline & & & & & & $\begin{array}{l}\text { Stage } \\
2\end{array}$ & 0.719 & 0.116 & 0.020 & $6.71 \cdot 10^{-9}$ & & CAD \\
\hline r 7137731 & 12002 & 12 & 95490999 & $\mathrm{~T}$ & $C$ & $\begin{array}{c}\text { Stage } \\
1 \\
\end{array}$ & 0.647 & -0.138 & 0.020 & $3.31 \cdot 10^{-12 *}$ & $F G D 6 / N R 2 C 1$ & - \\
\hline 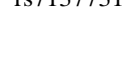 & $12 \mathrm{q} 22$ & & מים & & & $\begin{array}{c}\text { East } \\
\text { Asian }\end{array}$ & 0.640 & -0.086 & 0.026 & $1.01 \cdot 10^{-3}$ & 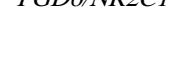 & \\
\hline & & & & & & $\begin{array}{l}\text { Stage } \\
2\end{array}$ & 0.644 & -0.119 & 0.016 & $4.88 \cdot 10^{-14}$ & & \\
\hline & & & & & & $\begin{array}{c}\text { Stage } \\
1\end{array}$ & 0.764 & -0.148 & 0.022 & $4.10 \cdot 10^{-11}$ & & \\
\hline rs 3742321 & $13 q 13.1 \dagger$ & 13 & 33704065 & $\mathrm{~T}$ & $\mathrm{C}$ & $\begin{array}{c}\text { East } \\
\text { Asian }\end{array}$ & 0.756 & -0.135 & 0.032 & $2.71 \cdot 10^{-5}$ & - & - \\
\hline & & & & & & $\begin{array}{c}\text { Stage } \\
2\end{array}$ & 0.762 & -0.144 & 0.018 & $5.47 \cdot 10^{-15}$ & & \\
\hline & & & & & & $\begin{array}{c}\text { Stage } \\
1\end{array}$ & 0.659 & -0.115 & 0.022 & $1.22 \cdot 10^{-7 *}$ & & Smoking \\
\hline rs8034191 & $15 \mathrm{q} 25.1$ & 15 & 78806023 & $\mathrm{~T}$ & $\mathrm{C}$ & $\begin{array}{l}\text { East } \\
\text { Asian }\end{array}$ & 0.976 & -0.161 & 0.091 & $7.69 \cdot 10^{-2}$ & PSMA4 & $\begin{array}{l}\text { behaviour, } \\
\text { lung } \\
\text { function }\end{array}$ \\
\hline & & & & & & $\begin{array}{l}\text { Stage } \\
2\end{array}$ & 0.676 & -0.117 & 0.021 & $2.75 \cdot 10-8$ & & COPD \\
\hline
\end{tabular}




\begin{tabular}{|c|c|c|c|c|c|c|c|c|c|c|c|c|}
\hline SNP & Locus & Chr & Position & $\mathbf{E A}$ & OA & Stage & EAF & beta & SE & P-value & $\begin{array}{l}\text { Annotated } \\
\text { genes }\end{array}$ & $\begin{array}{c}\text { Associated } \\
\text { traits }\end{array}$ \\
\hline \multirow{3}{*}{ rs7184525 } & \multirow{3}{*}{$16 q 23.1$} & \multirow{3}{*}{16} & \multirow{3}{*}{75437186} & \multirow{3}{*}{ A } & \multirow{3}{*}{ G } & $\begin{array}{c}\text { Stage } \\
1\end{array}$ & 0.450 & 0.148 & 0.023 & $8.80 \cdot 10^{-11 *}$ & \multirow{3}{*}{$\begin{array}{c}\text { BCAR1/ } \\
R P 11.252 K 23.2\end{array}$} & \multirow{3}{*}{-} \\
\hline & & & & & & $\begin{array}{l}\text { East } \\
\text { Asian }\end{array}$ & 0.459 & 0.123 & 0.028 & $1.04 \cdot 10^{-5}$ & & \\
\hline & & & & & & $\begin{array}{l}\text { Stage } \\
2\end{array}$ & 0.453 & 0.138 & 0.018 & $5.60 \cdot 10^{-15}$ & & \\
\hline \multirow{3}{*}{ rs11661542 } & \multirow{3}{*}{$18 \mathrm{q} 11.2 \dagger$} & \multirow{3}{*}{18} & \multirow{3}{*}{20223695} & \multirow{3}{*}{ A } & \multirow{3}{*}{$\mathrm{C}$} & $\begin{array}{c}\text { Stage } \\
1\end{array}$ & 0.516 & -0.166 & 0.021 & $5.74 \cdot 10^{-16}$ & \multirow{3}{*}{ - } & \multirow{3}{*}{-} \\
\hline & & & & & & $\begin{array}{c}\text { East } \\
\text { Asian }\end{array}$ & 0.401 & -0.087 & 0.026 & $6.82 \cdot 10^{-4}$ & & \\
\hline & & & & & & $\begin{array}{l}\text { Stage } \\
2\end{array}$ & 0.471 & -0.135 & 0.016 & $3.17 \cdot 10^{-17}$ & & \\
\hline \multirow{3}{*}{ rs4814863 } & \multirow{3}{*}{20 p11.23 } & \multirow{3}{*}{20} & \multirow{3}{*}{19469685} & \multirow{3}{*}{ A } & \multirow{3}{*}{ G } & $\begin{array}{c}\text { Stage } \\
1\end{array}$ & 0.248 & 0.096 & 0.024 & $6.71 \cdot 10^{-5}$ & \multirow{3}{*}{-} & \multirow{3}{*}{ - } \\
\hline & & & & & & $\begin{array}{l}\text { East } \\
\text { Asian }\end{array}$ & 0.513 & 0.110 & 0.025 & $1.10 \cdot 10^{-5}$ & & \\
\hline & & & & & & $\begin{array}{l}\text { Stage } \\
2\end{array}$ & 0.375 & 0.103 & 0.017 & $3.22 \cdot 10^{-9}$ & & \\
\hline \multirow{3}{*}{ rs39713 } & \multirow{3}{*}{$22 \mathrm{q} 12.1$} & \multirow{3}{*}{22} & \multirow{3}{*}{30343186} & \multirow{3}{*}{$\mathrm{T}$} & \multirow{3}{*}{$\mathrm{C}$} & $\begin{array}{c}\text { Stage } \\
1\end{array}$ & 0.088 & 0.182 & 0.033 & $4.10 \cdot 10^{-8}$ & \multirow{3}{*}{-} & \multirow{3}{*}{-} \\
\hline & & & & & & $\begin{array}{l}\text { East } \\
\text { Asian }\end{array}$ & $N A$ & $N A$ & $N A$ & $N A^{* *}$ & & \\
\hline & & & & & & $\begin{array}{l}\text { Stage } \\
2\end{array}$ & 0.088 & 0.182 & 0.033 & $4.10 \cdot 10^{-8}$ & & \\
\hline
\end{tabular}




\section{Table 2}

\section{SNP heritability estimates.}

Values are given on the observed scale $\left(\left(\mathrm{h}_{\mathrm{obs}}^{2}\right)\right)$ and liability scale $\left(\left(\mathrm{h}_{\text {liab }}^{2}\right)\right)$. Prevalence used for conversion to the liability scale is shown. Effective number samples was used for the conversion, as described in the Supplementary Note. For SumHer, two analyses were done: one with settings suggested by the SumHer authors, using LD reference data from the Health and Retirement Study (HRS) and one to mimic LDSC, with

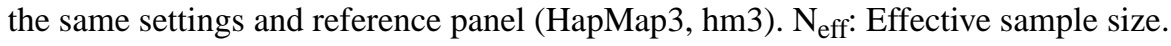

\begin{tabular}{|c|c|c|c|c|c|c|c|c|c|}
\hline Trait & Method & $\mathrm{h}_{\text {obs }}^{2}$ & SE $\left(\mathrm{h}_{\text {obs }}^{2}\right)$ & Prevalence & $\mathrm{h}_{\text {liab }}^{2}$ & $\mathbf{S E}\left(\mathrm{h}_{\text {liab }}^{2}\right)$ & Cases & Controls & $\mathbf{N}_{\text {eff }}$ \\
\hline $\begin{array}{c}\text { Intracranial aneurysms } \\
(\text { Stage 1) }\end{array}$ & LDSC & 0.295 & 0.038 & 0.03 & 0.216 & 0.028 & 7495 & 71934 & 24253 \\
\hline $\begin{array}{c}\text { Intracranial aneurysm } \\
\text { (Stage 1) }\end{array}$ & SumHer & 0.409 & 0.074 & 0.03 & 0.299 & 0.054 & 7495 & 71934 & 24253 \\
\hline $\begin{array}{c}\text { Intracranial aneurysm } \\
\text { (Stage 1) }\end{array}$ & $\begin{array}{c}\text { SumHer } \\
\text { (LDSC) }\end{array}$ & 0.276 & 0.037 & 0.03 & 0.202 & 0.027 & 7495 & 71934 & 24253 \\
\hline aSAH-only & LDSC & 0.296 & 0.043 & 0.005 & 0.140 & 0.020 & 5140 & 71952 & 17019 \\
\hline uIA-only & LDSC & 0.393 & 0.075 & 0.03 & 0.223 & 0.044 & 2070 & 71952 & 7721 \\
\hline
\end{tabular}

